\title{
Catalytic Nanoassemblies Formed by Short Peptides Promote Highly Enantioselective Transfer Hydrogenation
}

\section{SUPPORTING INFORMATION}

Martin A. Dolan, Prem N. Basa, Oleksii Zozulia, Zsófia Lengyel, René Lebl, Eric M. Kohn, Sagar Bhattacharya and Ivan V. Korendovych*

1-014 Center for Science and Technology, Department of Chemistry, Syracuse University, Syracuse, NY 13244

E-mail: ikorendo@syr.edu 


\section{General Procedures and Instrumentation}

${ }^{1} \mathrm{H}$ and ${ }^{13} \mathrm{C}$ NMR. All spectra were recorded on a Bruker Avance DPX-300 (300 MHz) spectrometer, a Bruker Avance DPX-400 (400 MHz) spectrometer, or a Bruker Avance III HD (800 MHz) spectrometer. Chemical shifts are reported in parts per million (ppm) and are calibrated using residual undeuterated solvent as an internal reference $\left(\mathrm{CDCl}_{3}\right.$ : $7.26 \mathrm{ppm}$; DMSO-d 6 : $2.50 \mathrm{ppm}$ ). Data are reported as follows: chemical shift, multiplicity, coupling constants $(\mathrm{Hz})$, and integration. The following abbreviations or combinations thereof were used to note signal multiplicities: $s=$ singlet, $d=$ doublet, $t=$ triplet, $q=$ quartet, $p=$ pentet, sext $=$ sextet, $\mathrm{m}=$ multiplet, $\mathrm{dd}=$ doublet of doublets, ddd =doublet of doublet of doublets, $\mathrm{br}=$ broad. ${ }^{13} \mathrm{C}$ NMR spectra were recorded on a Bruker Avance DPX-300 (75 MHz) spectrometer or a Bruker Avance DPX-400 (100 MHz) spectrometer with complete proton decoupling. Chemical shifts are reported in ppm and are calibrated using residual undeuterated solvent as an internal reference $\left(\mathrm{CDCl}_{3}: \delta 77.23 \mathrm{ppm}\right.$; DMSO-d 6 : $39.52 \mathrm{ppm})$.

HPLC. Enantiomeric excess was determined by chiral HPLC using a (250 x $4.6 \mathrm{~mm})$ Phenomenex Lux-Cellulose 1 on a Shimadzu Prominence UFLC instrument. The enantiomeric selectivity was estimated by integration of signals at $254 \mathrm{~nm}$. All solvents used in our normal-phase system contained $0.1 \%$ TFA. The conditions for separation of alcohol enantiomers can be found in the compound characterization of transfer hydrogenation products.

GC. Enantiomeric excess was also determined by chiral GC using a (30 m x $0.25 \mathrm{~mm}$ x $0.25 \mu \mathrm{m}$ ) Agilent J\&W Cyclodex-B column on an Agilent 7820A instrument. The conditions for separation of alcohol enantiomers can be found in the compound characterization of transfer hydrogenation products.

IR spectra. Infrared (IR) spectra were recorded on a Thermo Fischer Scientific Nicolet IR-100 or Nicolet iS5 spectrometer, $v$ max in $\mathrm{cm}^{-1}$, and were obtained from samples prepared as pellets in $\mathrm{KBr}$, oils with $\mathrm{NaCl}$ plates, or neat. 
Melting point. Melting points (m.p.) are uncorrected and were recorded using an Electrothermal Mel-Temp melting point apparatus.

MALDI-TOF. A Bruker Autoflex III Smartbeam MALDI-TOF mass spectrometer was used for identification of the peptides.

Electrospray ionization (ESI). ESI mass spectrometry analyses were carried out on Shimadzu LCMS-8100.

DFT Modeling. All simulations were carried out with the Gaussian 16 Rev. B.01 software package. ${ }^{1}$ The geometries of the structures were optimized with density functional theory (DFT) employing the parameter-free Perdew-Burke-Ernzerhof (PBE0) hybrid functional ${ }^{2}$ and the valence triple-zeta polarization basis set (def2-TZVP) for all atoms. ${ }^{3}$ Convergence cutoffs on forces and step sizes were tightened using the keyword, opt=tight, and the integration grid was set to 99 radial shells and 590 angular points per shell with the keyword, integral (grid=ultrafine).

Transmission Electron Microscopy (TEM). TEM measurements were performed on a JEOL 2000EX instrument operated at $120 \mathrm{kV}$ with a tungsten filament at N.C. Brown Center for Ultrastructure studies at SUNY-ESF. The particle size was analyzed manually by modeling each self-assembled structure as a sphere.

Sample Preparation: A stock solution of ${ }^{\mathrm{PPL}-\mathrm{C}_{16}}$ with $\left[\mathrm{IrCp}^{*} \mathrm{Cl}_{2}\right]_{2}$ with $25 \mathrm{mM}$ peptide and $12.5 \mathrm{mM}$ metal was made in $20 \%$ TFE and $3.125 \mathrm{M}$ sodium formate in milli-Q water. A diluted $50 \mu \mathrm{M}$ solution was prepared by transferring $8 \mu \mathrm{L}$ of stock solution and diluting to $4 \mathrm{~mL}$ with $20 \% \mathrm{TFE} / \mathrm{H}_{2} \mathrm{O}$. A $10 \mu \mathrm{L}$ aliquot of sample was adsorbed for 5 minutes onto a formvar/carbon-coated, 200-mesh copper grid. The grid was blotted with filter paper to draw out excess liquid. A drop of $2 \%(\mathrm{w} / \mathrm{v})$ uranyl acetate stain was added to the grid that was then immediately blotted. An additional $50 \mu \mathrm{L}$ of $2 \%(\mathrm{w} / \mathrm{v})$ uranyl acetate was added and adsorbed for 2 minutes. The grid was washed by dipping into milli-Q water several times, blotted, and finally dried overnight under vacuum. 
Dynamic Light Scattering (DLS). DLS measurements were performed on a Malvern Zetasizer Nano ZS instrument utilizing a $173^{\circ}$ backscattering detector. The hydrodynamic diameter $\left(D_{h}\right)$ and $Z$-average $\left(Z_{\text {ave }}\right)$ were calculated using CONTIN analysis.

Sample preparation:

Catalyst in TFE $/ \mathrm{H}_{2} \mathrm{O}$. Stock solutions of ${ }^{\mathrm{PPL}}-\mathrm{C}_{16}$ and $\left[\mathrm{IrCp}^{*} \mathrm{Cl}_{2}\right]_{2}$ were made at $25 \mathrm{mM}$ peptide and $12.5 \mathrm{mM}$ metal, respectively in TFE. A $0.02 \mathrm{mM}$ sample were prepared by transferring $0.8 \mu \mathrm{L}$ of the peptide and metal stock solution into $199 \mu \mathrm{L}$ of TFE followed by addition of $800 \mu \mathrm{L}$ of a $3.125 \mathrm{M}$ sodium formate solution in milli-Q water to give $\mathrm{Cp} * \operatorname{lr}(\mathrm{H})\left({ }^{\mathrm{DPL}}-\mathrm{C}_{16}\right)$ in $20 \% \mathrm{TFE} / \mathrm{H}_{2} \mathrm{O}$.

Ligand. A $25 \mathrm{mM}$ peptide stock solution of ${ }^{\mathrm{PPL}-\mathrm{C}_{16}}$ was made in TFE. A $0.02 \mathrm{mM}$ sample was prepared by transferring $0.8 \mu \mathrm{L}$ of the peptide stock solution into $199 \mu \mathrm{L}$ of TFE followed by addition of $800 \mu \mathrm{L}$ of milli-Q water to give ${ }^{\mathrm{PPL}}-\mathrm{C}_{16}$ in $20 \% \mathrm{TFE} / \mathrm{H}_{2} \mathrm{O}$.

Catalyst in $\mathrm{EtOH} / \mathrm{H}_{2} \mathrm{O}$. A stock solution of ${ }^{\mathrm{DPL}-\mathrm{C}_{16}}$ with $\left[\mathrm{IrCp}^{*} \mathrm{Cl}_{2}\right]_{2}$ was made at $25 \mathrm{mM}$ peptide and $12.5 \mathrm{mM}$ metal in ethanol. Samples of $50 \mu \mathrm{M}, 75 \mu \mathrm{M}$, and $100 \mu \mathrm{M}$ $\mathrm{Cp}^{*} \operatorname{Ir}(\mathrm{H})\left({ }^{\mathrm{D} P \mathrm{~L}}-\mathrm{C}_{16}\right)$ concentrations were prepared by transferring $2 \mu \mathrm{L}, 3 \mu \mathrm{L}$, and $4 \mu \mathrm{L}$, respectively, of peptide and metal stock solution into separate glass vials. Each vial was filled to $200 \mu \mathrm{L}$ total volume of ethanol, followed by addition of $800 \mu \mathrm{L}$ with a $3.125 \mathrm{M}$ sodium formate solution in milli-Q water to give $\mathrm{Cp}{ }^{*} \operatorname{Ir}(\mathrm{H})\left({ }^{\mathrm{DPL}}-\mathrm{C}_{16}\right)$ in $20 \% \mathrm{EtOH} / \mathrm{H}_{2} \mathrm{O}$.

Single crystal X-ray diffraction. Crystals of DPF- $\mathrm{C}_{12}$ (Fig 2D) were grown by slow evaporation in a MeOH:DCM (2:8) solution to yield colorless needles. Single crystal structural analysis was carried out using a Bruker KAPPA APEX DUO diffractometer equipped with APEX II CCD and LT-II low temperature device. The diffraction data were collected at $100 \mathrm{~K}$ using $\mathrm{Cu}-\mathrm{K}_{\alpha}$ radiation using the omega scan technique. The unit cell and space group were determined using the SAINT+ program. The structure was solved using SHELXL-2014/719. All non-hydrogen atoms were refined anisotropically. Hydrogen 
atoms were positioned based on electron density within the refined solved structure. The structure has been deposited into CCDC (1884477).

Gel filtration. A fluorophore mixture containing terbium (III) chloride hexahydrate $\left(\mathrm{TbCl}_{3} \cdot 6 \mathrm{H}_{2} \mathrm{O}\right)$ and pyridine-2,6-dicarboxylic acid (DPA) was prepared in milli-Q water to make a $9 \mathrm{~mL} \mathrm{TbCl} / \mathrm{DPA}$ solution. To this $9 \mathrm{~mL}$ fluorophore solution was added $1 \mathrm{~mL}$ of 200 mM HEPES buffer ( $\mathrm{pH}$ 7.0) where the final concentrations of $\mathrm{TbCl}_{3}$ and DPA were 1 $\mathrm{mM}$ and $2 \mathrm{mM}$ respectively with $20 \mathrm{mM}$ HEPES ( $\mathrm{pH}$ 7.0). Following a similar preparation method, solid sodium formate was added to $9 \mathrm{~mL}$ of $\mathrm{TbCl}_{3} / \mathrm{DPA}$ fluorophore solution, vortexed until fully dissolved, and then diluted to $10 \mathrm{~mL}$ with milli-Q water to give the final concentrations of $\mathrm{TbCl}_{3}$ and DPA at $1 \mathrm{mM}$ and $2 \mathrm{mM}$, respectively, with sodium formate at $3.75 \mathrm{M}$.

Preparation of 1-palmitoyl-2-oleoyl-glycero-3-phosphocholine (POPC) vesicles. $25 \mathrm{mg}$ of solid POPC (Avanti Polar Lipids) was dissolved in $1 \mathrm{~mL}$ of TFE to make a lipid stock of $33 \mathrm{mM}$. $140 \mu \mathrm{L}$ of $33 \mathrm{mM}$ stock was pipetted into a glass test tube and the solvent was evaporated under a stream of nitrogen. The resulting films were dried in vacuo for $15 \mathrm{~min}$. $1 \mathrm{~mL}$ of the solution containing $1 \mathrm{mM}$ fluorophore $\left(\mathrm{Tb}(\mathrm{DPA})_{2}\right)$ and $3.125 \mathrm{M}$ sodium formate was added to the dried POPC lipid film (final lipid concentration of $4.6 \mathrm{mM}$ ) and vortexed for $10 \mathrm{~min}$ followed by tip sonication (30 s) in a $1.5 \mathrm{~mL}$ Eppendorf tube. Alternatively, to get better benchmark values for the vesicles elution time the same procedure was done with 50 mM HEPES pH 7.0 instead of sodium formate.

Preparation of $C p^{*} / r(H)\left({ }^{D} P L-C_{16}\right)$ vesicles. In a glass vial, ${ }^{D P L}-C_{16}(11.3 \mathrm{mg})$ and $\left[\mathrm{IrCp}^{*} \mathrm{Cl}_{2}\right]_{2}(5.0 \mathrm{mg})$ were dissolved in $200 \mu \mathrm{L}$ TFE. $800 \mu \mathrm{L}$ of the fluorophore solution $\left(1 \mathrm{mM} \mathrm{Tb}(\mathrm{DPA})_{2}\right.$ and $3.125 \mathrm{M}$ sodium formate) was transferred to the glass vial.

Gel filtration. $1 \mathrm{~mL}$ of the solution containing vesicles was loaded onto a gel filtration column (Sepharose CL-4B resin, $9 \mathrm{~cm}$ long $\times 2 \mathrm{~cm}$ in diameter) pre-equilibrated with 20 mM HEPES ( $\mathrm{pH} 7.0$ ) or $3 \mathrm{M}$ sodium formate buffers (as appropriate) followed by elution of $0.5 \mathrm{~mL}$ fractions. The fluorescence of the eluted fractions were measured on a 
fluorescent platereader (SpectraMax Gemini) using excitation and emission wavelengths of $270 \mathrm{~nm}$ and $490 \mathrm{~nm}$, respectively.

\section{Ligand syntheses}

Unless otherwise indicated, all reactions were conducted in oven- $\left(140^{\circ} \mathrm{C}\right)$ or flame-dried glassware. Yields refer to chromatographically and spectroscopically ( $\left.{ }^{1} \mathrm{H} N M R\right)$ homogeneous materials, unless otherwise stated. Flash column chromatography was performed using Silia Flash ${ }^{\circledR}$ P60 silica gel $(40-63 \mu \mathrm{m})$ from Silicycle, Brockmann Grade I neutral alumina (50-200 $\mu \mathrm{m})$ from Acros Organics, or Brockmann Grade I basic alumina (58 $\AA$ ) from Alfa Aesar. All work-up and purification procedures were carried out with reagent grade solvents (purchased from VWR or Pharmaco-Aaper). The identities of the purified peptides were confirmed either by ${ }^{1} \mathrm{H}$ NMR, Bruker Autoflex III Smartbeam MALDI-TOF mass spectrometer, or by mass spectrometry. The purity of the peptides were determined by NMR.

\section{Solid-Phase Peptide Synthesis}

Dipeptides were synthesized by manual fluorenylmethyloxycarbonyl (Fmoc) solid phase peptide synthesis strategy as described before. ${ }^{4}$ Deprotection of the side chains and cleavage of the peptides from the rink-amide MBHA resin was achieved by subjecting the peptides to a mixture of TFA/ $\mathrm{H}_{2} \mathrm{O} / \mathrm{TIS}$ (95: 2.5: 2.5, v/v), or TFA/ $\mathrm{H}_{2} \mathrm{O} / \mathrm{TIS} / \mathrm{EDT}$ (93: 2.5: 2.5: $2, \mathrm{v} / \mathrm{v}$ for oxidation sensitive functionalities) for 2 hours at room temperature. The solution was then filtered through a pipette plugged with glass wool and the solution concentrated to remove excess TFA using a stream of nitrogen. Purification of short dipeptides with C-terminal amides can easily be done by dissolving it in a minimum amount of TFA and transferring the solution into dry-ice cooled diethyl ether to facilitate precipitation. The precipitated peptide was then spun down using a centrifuge, the ether discarded, and then dried under a vacuum pump. If the peptides were not easily crashed out with diethyl ether, the concentrated solution was diluted with water or alternatively a mixture of solvent $A\left(99.9: 0.1 \mathrm{H}_{2} \mathrm{O} /\right.$ TFA $)$ and solvent $\mathrm{B}\left(90: 9.9: 0.1 \mathrm{MeCN} / \mathrm{H}_{2} \mathrm{O} / \mathrm{TFA}\right)$ to ca. $5 \mathrm{~mL}$ total volume. The homogeneous solutions were then subjected to lyophilization. 
This cycle was repeated until lyophilization afforded peptide trifluoroacetates as white fluffy solids.

\section{Liquid Phase Peptide Synthesis}

Short peptides with C-terminal alkyl tails were synthesized by solution-phase synthesis utilizing the tertbutyloxycarbonyl (Boc) peptide synthesis strategy using DMF as the reaction solvent. A slight excess of the activator HBTU $\left(N, N, N^{\prime}, N^{\prime}\right.$-tetramethyl-O- $(1 \mathrm{H}-$ benzotriazol-1-yl)uronium hexafluorophosphate, or its analog HCTU 2-(6-chloro-1-Hbenzotriazole-1-yl)-1,1,3,3-tetramethylaminium hexafluorophosphate), were used in our peptide syntheses. In general, the alkyl amine was also added in a similar molar ratio to activator, with 2 equivalents of base $N, N^{\prime}$-diisopropylethyl amine (DIPEA) added.

\section{Asymmetric Transfer Hydrogenation (ATH) Reactions}

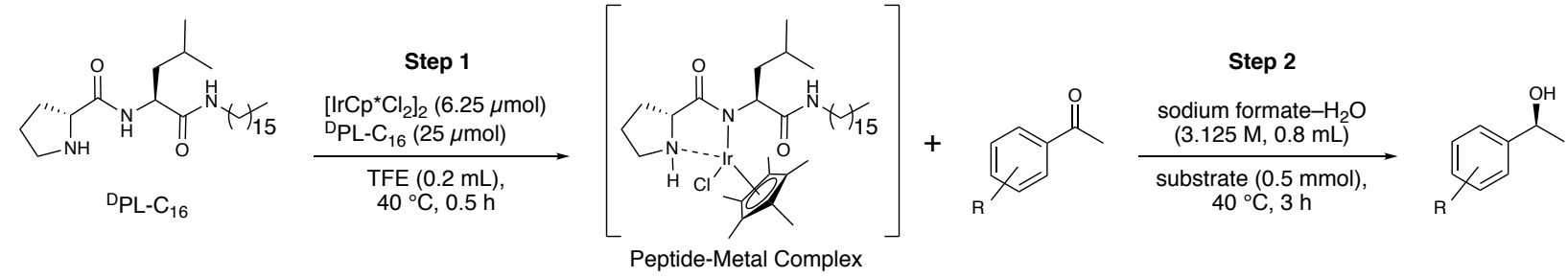

Scheme S1: Standard ATH Conditions

\section{ATH Method 1: Single Reaction}

To an oven-dried tapered glass microtube ${ }^{\mathrm{PPL}-\mathrm{C}_{16}}(11.3 \mathrm{mg}, 25 \mu \mathrm{mol}),\left[\mathrm{IrCp}^{*} \mathrm{Cl}_{2}\right]_{2}(5.0$ $\mathrm{mg}, 6.25 \mu \mathrm{mol}$ ), and $0.2 \mathrm{~mL}$ trifluoroethanol (TFE) were added. The tube was sealed with a 14/20 rubber septum and a short needle was then inserted through the rubber septum into the head space. The microtube was agitated (1000-1200 rpm) for 30 minutes at $40^{\circ} \mathrm{C}$ in a Thermo-Mixer shaker (Fisher Scientific). After formation of the pre-catalyst/peptidemetal complex, $0.8 \mathrm{~mL}$ of a prepared $3.125 \mathrm{M}$ sodium formate stock solution in water was added followed by addition of substrate $(0.5 \mathrm{mmol})$. The tube is resealed with the rubber septum and needle, and is placed back in the Thermo-Mixer and is agitated (1000-1200 rpm) for 3 hours at $40^{\circ} \mathrm{C}$. 


\section{ATH Method 2: Multiple Reaction Setup}

Note: This method allows for scalability and a pre-calculated stock solution can be created for multiple ATH reactions to be run in parallel. Due to some observed minimal evaporation of TFE after heating, an additional $5 \%$ amount of reagents and solvent are included into the calculated values to compensate for the small loss of TFE.

In a typical reaction, DPL-C 16 (11.3 mg, $25.0 \mu \mathrm{mol} \times$ (\# of reactions $+5 \%$ excess), $\left[\mathrm{IrCp}^{*} \mathrm{Cl}_{2}\right]_{2}(5.0 \mathrm{mg}, 6.25 \mu \mathrm{mol} \times$ (\# of reactions $+5 \%$ excess), and $0.2 \mathrm{~mL}$ TFE $\times$ (\# of reactions $+5 \%$ excess) were added to a $15 \mathrm{~mL}$ screw-capped tube equipped with a magnetic stir bar. The tube was then sealed and immersed into a pre-heated $40{ }^{\circ} \mathrm{C}$ oil bath and the pre-catalyst solution was stirred for 30 minutes. The tube was then removed from the oil bath and allowed to cool to room temperature. Aliquots of the solution (0.2 $\mathrm{mL}$ ) are then transferred to separate glass-tapered microtubes followed by addition of a prepared $3.125 \mathrm{M}$ sodium formate solution in water $(0.8 \mathrm{~mL})$, and substrate $(0.5 \mathrm{mmol})$. The microtubes were then sealed with a 14/20 rubber septum and a short needle was inserted through the rubber septum into the head space to vent $\mathrm{CO}_{2}$ formation and limit reaction mixture evaporation. The microtube(s) were agitated (1000-1200 rpm) for 3 hours at $40{ }^{\circ} \mathrm{C}$ in a Thermo-Mixer shaker.

\section{Work Up Method 1: Extraction}

The crude reaction mixture was transferred to a separatory funnel (the reaction tube was rinsed with either dichloromethane (DCM) or methyl-tert-butyl ether (MTBE) and washes were combined with the reaction mixture). The organics were extracted from the aqueous layer with either DCM or MTBE $(2 \times 5 \mathrm{~mL})$. The combined organics were washed with saturated brine ( $5 \mathrm{~mL}$ ), dried over $\mathrm{Na}_{2} \mathrm{SO}_{4}$ or $\mathrm{MgSO}_{4}$, filtered through a fritted funnel, and the solvent removed under reduced pressure on a rotavap to afford the alcohol product.

\section{Work Up Method 2: Silica Plug Purification}

For quick analysis of results, the crude reaction mixture is directly purified through a short silica plug (ca. $2.0 \mathrm{~cm}$ height) by eluting with MTBE and collecting $50-100 \mathrm{~mL}$ of solvent. Note 1: Due to the amount of water in the reaction mixture, some silica has been observed 
to accumulate in the collection flask. Note 2: This method was not applied with pyridine substrates.

Yields were determined by ${ }^{1} \mathrm{H}$ NMR with the addition of mesitylene as an internal standard ( $6.8 \mathrm{ppm}, 1 \mathrm{H}$ and $\sim 2.3 \mathrm{ppm}, 3 \mathrm{H})$ in $\mathrm{CDCl}_{3}$.

$$
\frac{0.5 \mathrm{mmol} \text { (substrate) }}{3 \text { (symmetrical signal intensity) }} \times \frac{120.19 \mathrm{~g}}{\mathrm{~mol}} \times \frac{1 \mathrm{~mL}}{0.869 \mathrm{~g}}=23.1 \mu \mathrm{L}
$$

Enantiomeric excess was then determined either by HPLC or GC. Knowing the approximate yields by ${ }^{1} \mathrm{H}$ NMR first, provided us useful information on how much to dilute the products. For HPLC, the products are dissolved in 10:90 EtOH:Hexanes, the volume of which is closely based on the ${ }^{1} \mathrm{H}$ NMR yields (e.g. $0.7 \mathrm{~mL}$ of the EtOH:Hexanes will be added to a $\sim 72 \%$ yield reaction). This initial solution is then diluted 10 -fold by taking an aliquot of $100 \mu \mathrm{L}$ and transferring to a glass culture tube and then adding $900 \mu \mathrm{L}$ of hexanes. Approximately $200 \mu \mathrm{L}$ of diluted solution are transferred to an eppendorf tube equipped with a 0.44 micron filter and spun down in a VWR® C1213 Galaxy Mini Centrifuge for at least 15 seconds. The filtered solution is transferred to an HPLC vial fitted with a glass insert and analyzed using chiral HPLC as described above. For GC, the products were instead dissolved with DCM (e.g. $4 \mathrm{~mL}$ will be added to a $\sim 44 \%$ yield reaction) and ca. $2 \mu \mathrm{L}$ of the diluted solution was then manually injected into a chiral capillary GC column under isothermal conditions.

All racemic alcohols were synthesized from their corresponding ketones using sodium borohydride. Standard reaction conditions involved substrate $(1 \mathrm{mmol})$ dissolved in $2 \mathrm{~mL}$ EtOH with addition of $\mathrm{NaBH}_{4}(1.5 \mathrm{mmol})$ and was reacted for 2-3 hours. The reaction mixture was quenched with water $(5 \mathrm{~mL})$ transferred to a separatory funnel, and the organics were extracted with EtOAc $(2 \times 25 \mathrm{~mL})$, washed with saturated brine, dried over $\mathrm{MgSO}_{4}$, filtered, and concentrated to give the corresponding oil or solid. 


\section{Synthesis of Tailed Dipeptides}

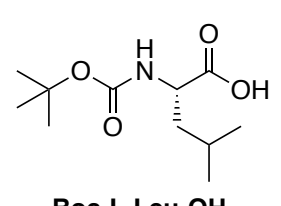

Boc-L-Leu-OH
1) HBTU or HCTU (1.1 equiv.)

DIPEA ( 2 equiv.)

alkylamine (1.1 equiv.) DMF, RT, $1 \mathrm{~h}$

2) TFA (>50 equiv.), RT, $0.25 \mathrm{~h}$

3) $\mathrm{NaHCO}_{3}(\mathrm{aq}) \mathrm{pH}=7$

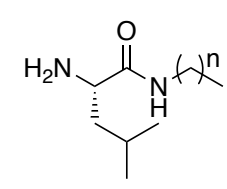

$\mathrm{n}=7,9,11,13,15,17$

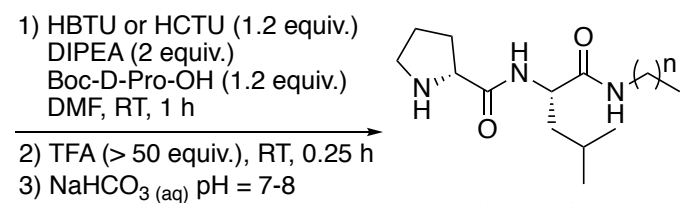

$\mathrm{n}=7,9,11,13,15,17$

Scheme S2: Synthesis of ${ }^{D} P L-C_{n}$ peptides

\section{General Protocol A: $\mathrm{H}_{2} \mathrm{~N}-$ Leu- $\mathrm{C}_{\mathrm{n}}(\mathrm{n}=\mathbf{8 - 1 8})$}

To the flask containing Boc-L-Leucine $(575 \mathrm{mg}, 2.5 \mathrm{mmol}$ ) was added alkylamines (2.0$2.2 \mathrm{mmol})$, and HCTU (1.24 g, $3.0 \mathrm{mmol})$. The solids were dissolved in $25 \mathrm{~mL}$ of DMF. Next, DIPEA $(0.52 \mathrm{~mL}, 3.0 \mathrm{mmol})$ was then added and the contents were stirred at room temperature for one hour. The solution was then transferred to a separatory funnel. The round bottom flask was rinsed three times with EtOAc (50 mL total) and then added to the separatory funnel. The organics were extracted from the aqueous layer using EtOAc $(2 \times 75 \mathrm{~mL})$. The combined organics $(200 \mathrm{~mL}$ in total) were washed with water $(100 \mathrm{~mL})$, saturated brine $(3 \times 100 \mathrm{~mL})$, dried over $\mathrm{MgSO}_{4}$, filtered over a fritted funnel and concentrated to afford a crude Boc protected intermediate. Next, to the flask containing crude Boc-Leu- $C_{n}$ was added magnetic stirring bar and trifluoroacetic acid (ca. $15 \mathrm{~mL}$ ). The solution was then stirred for 15 minutes. The TFA was removed by blowing air over it until a viscous oil remained. The excess TFA was then neutralized with a saturated $\mathrm{NaHCO}_{3}$ solution $(100 \mathrm{~mL}, \mathrm{pH}=7)$. The solution was then transferred to a separatory funnel. The organics were extracted from the aqueous layer using EtOAc $(2 \times 75 \mathrm{~mL})$. The combined organics ( $200 \mathrm{~mL}$ in total) were washed with saturated brine $(3 \times 100 \mathrm{~mL})$ and dried over $\mathrm{MgSO}_{4}$, filtered over a fritted funnel and concentrated to afford crude material. Silica gel column chromatography was then performed. A 1:1 EtOAc:hexanes eluent is used to remove impurities, then the solvent is switched to DCM, and the product band was eluted using 5\% methanol in DCM mixture. Upon solvent evaporation, colorless oils to white semisolids were obtained. 


\section{General Protocol B: DPL- $C_{n}(n=8-18)$ Peptides}

To the flask containing Boc- ${ }^{-}$Pro-COOH $(0.43 \mathrm{~g}, 2.00 \mathrm{mmol})$, was added Leu- $\mathrm{C}_{\mathrm{n}}(1.78$ $\mathrm{mmol})$, HCTU (0.83 g, $2.0 \mathrm{mmol})$, DIPEA $(0.52 \mathrm{~mL}, 3.0 \mathrm{mmol})$ and $25 \mathrm{~mL}$ of DMF. The contents were stirred at room temperature for one hour. The solution was then transferred to a separatory funnel. The solution was diluted with saturated brine solution and the organics were extracted using EtOAc (150 mL total). The organics were separated, and the combined organics portions were washed with water $(100 \mathrm{~mL})$, saturated brine $(3 \mathrm{x}$ $100 \mathrm{~mL}$ ), dried over $\mathrm{MgSO}_{4}$, filtered over a fritted funnel and concentrated to afford crude solid. To the flask containing crude Boc- ${ }^{-}$Pro-Leu- $C_{n}$ was added a magnetic stirring bar and trifluoroacetic acid (ca. $10 \mathrm{~mL}$ ). The solution was then stirred for 15 minutes at room temperature. TFA was then removed by blowing air over it until a viscous oil remained. The excess TFA was then neutralized with a saturated $\mathrm{NaHCO}_{3}$ solution $(100 \mathrm{~mL}, \mathrm{pH}=$ 7-8). The solution was then transferred to a separatory funnel. The round bottom flask was then rinsed with EtOAc ( $50 \mathrm{~mL}$ total) and the solution was added to the separatory funnel. The organics were separated, and then the organics were extracted from the aqueous layer using EtOAc $(2 \times 75 \mathrm{~mL})$. The combined organics (200 $\mathrm{mL}$ in total) were washed with saturated brine, dried over $\mathrm{MgSO}_{4}$, filtered over a fritted funnel and solvents were removed under reduced pressure. Alumina (basic or neutral) column chromatography was then performed. A 1:1 EtOAc:hexanes eluent is used to remove impurities, then the solvent is switched to DCM, and the product band was eluted using $5 \%$ methanol in DCM mixture. Upon solvents evaporation, colorless to white solids were obtained. 


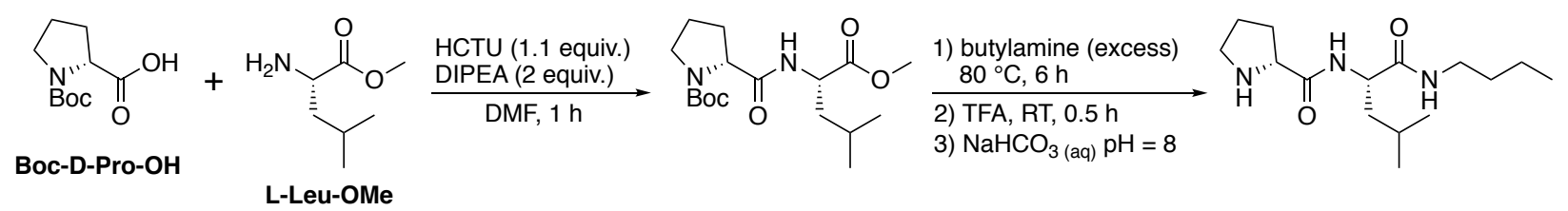

Scheme S3. Synthetic scheme for preparation of ${ }^{D} P L-C_{4}$.

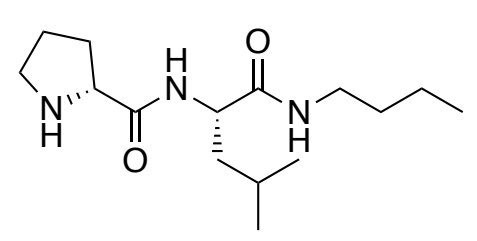

\section{${ }^{D P L}-C_{4}$}

To a round bottom flask equipped with a magnetic stirring bar was added, Boc- ${ }^{-}$Pro-COOH (1.07 g, $5 \mathrm{mmol}$ ), L-Leu-methyl ester $(1.09 \mathrm{~g}, 6 \mathrm{mmol}), \mathrm{HCTU}(2.48 \mathrm{~g}, 6 \mathrm{mmol})$ and $25 \mathrm{~mL}$ DMF followed by addition of DIPEA $(1.9 \mathrm{ml}, 10 \mathrm{mmol})$. The contents were stirred at room temperature for one hour and then the solution was transferred to a separatory funnel. The solution was diluted with saturated brine and the organics were extracted several times with EtOAc (100 mL total). The organics were separated, and the combined organics portions were dried over $\mathrm{MgSO}_{4}$, filtered over a fritted funnel and concentrated to afford crude solid. Purification of the intermediate following protocol B using basic alumina afforded a white crystalline solid $(0.56 \mathrm{~g}, 33 \%$ yield $)$ that was carried over to the next step of the syntheses.

To the flask containing Boc-DPro-LLeu-OMe $(1.0 \mathrm{~g}, 3.0 \mathrm{mmol})$, excess butylamine $(5.0 \mathrm{~mL}, 50.6 \mathrm{mmol})$ was added and heated at $80^{\circ} \mathrm{C}$ for 6 hours in double-walled sealed glass tube. The tube was allowed to cool to room temperature and then the glass tube was rinsed thoroughly using DCM $(15 \mathrm{~mL})$. Solvents were removed in vacuo to yield a white solid. TFA (ca. $5 \mathrm{~mL}$ ) was added and the solution was then stirred for 0.5 hours under ambient conditions in air. TFA was then removed by blowing air over it until a viscous oil remained. The residual TFA was then neutralized with saturated $\mathrm{NaHCO}_{3}(\mathrm{pH}$ $=8$ ). The solution was then transferred to a separatory funnel and extracted several times with EtOAc (100 mL total). The combined organics were washed with saturated brine, dried over $\mathrm{MgSO}_{4}$, filtered over a fritted funnel and concentrated to afford a colorless solid $(0.31 \mathrm{~g}, 38 \%$ yield $)$.

${ }^{1} \mathrm{H}-\mathrm{NMR}\left(300 \mathrm{MHz}, \mathrm{d}_{6}-\mathrm{DMSO}\right) \delta=7.99-7.92(\mathrm{~m}, 2 \mathrm{H}), 4.30-4.22(\mathrm{~m}, 1 \mathrm{H}), 3.53(\mathrm{dd}, J=8.4$, $4.8 \mathrm{~Hz}, 1 \mathrm{H}), 3.12-2.95(\mathrm{~m}, 2 \mathrm{H}), 2.88-2.71(\mathrm{~m}, 2 \mathrm{H}), 1.98-1.87(\mathrm{~m}, 1 \mathrm{H}), 1.64-1.19(\mathrm{~m}, 11 \mathrm{H})$, $0.88-0.83(\mathrm{~m}, 9 \mathrm{H})$ 
${ }^{13}$ C-NMR $\left(100 \mathrm{MHz}, \mathrm{d}_{6}\right.$-DMSO) $\delta=173.8,171.6,60.1,50.5,46.6,41.9,38.0,31.1,30.4$, 25.7, 24.3, 22.9, 21.9, 19.4, 13.6.

FT-IR: (neat) v = $3275(\mathrm{~m}), 3091(\mathrm{w}), 2955(\mathrm{~m}), 2933(\mathrm{~m}), 2863(\mathrm{w}), 1636(\mathrm{~s}), 1541(\mathrm{~s})$, $1227(\mathrm{~m}), 706(\mathrm{~m}) \mathrm{cm}^{-1}$

LC-MS (ESI): expected $\mathrm{m} / \mathrm{z}$ : [M + H] $]^{+}, 284.23$ and observed 284.20

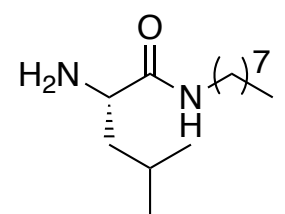

\section{$\mathrm{H}_{2} \mathrm{~N}-$ Leu-C $_{8}$}

Following general protocol A, Boc-L-Leucine $(1.15 \mathrm{~g}, 5.0 \mathrm{mmol})$, 1octylamine $(0.51 \mathrm{~g}, 4.0 \mathrm{mmol})$, HCTU $(2.06 \mathrm{~g}, 5.0 \mathrm{mmol})$ were dissolved in $25 \mathrm{~mL}$ of DMF followed by addition of DIPEA (1.04 mL, $6.0 \mathrm{mmol})$. Purification afforded a colorless oil $(0.72 \mathrm{~g}, 74 \%$ overall yield).

${ }^{1} \mathrm{H}-\mathrm{NMR}\left(400 \mathrm{MHz}, \mathrm{CDCl}_{3}\right) \delta=7.20(\mathrm{br}, 1 \mathrm{H}), 3.42-3.39(\mathrm{~m}, 1 \mathrm{H}), 3.20(\mathrm{q}, J=7.3 \mathrm{~Hz}, 2 \mathrm{H})$, 2.20 (br, 2H), 1.75-1.63 (m, 2H), $1.48(\mathrm{p}, J=7.0 \mathrm{~Hz}, 2 \mathrm{H}), 1.37-1.25(\mathrm{br}, 11 \mathrm{H}), 0.94(\mathrm{~d}, J$ $=6.3 \mathrm{~Hz}, 3 \mathrm{H}), 0.91(\mathrm{~d}, J=6.2 \mathrm{~Hz}, 3 \mathrm{H}), 0.87(\mathrm{t}, J=6.7 \mathrm{~Hz}, 3 \mathrm{H})$

${ }^{13} \mathrm{C}-\mathrm{NMR}\left(100 \mathrm{MHz}, \mathrm{CDCl}_{3}\right) \delta=175.1,53.5,44.0,39.2,31.8,29.6,29.3(2 \mathrm{C}), 27.0,24.9$, 23.3, 22.7, 21.6, 14.1

FT-IR (NaCl): 3298, 2955, 2926, 2855, 1649, 1538, 1467, 1368, $846 \mathrm{~cm}^{-1}$

LC-MS (ESI): expected $\mathrm{m} / \mathrm{z}:[\mathrm{M}+\mathrm{H}]^{+}, 243.42$ and observed 243.20

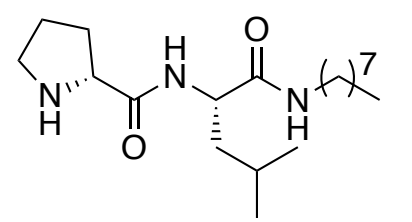

${ }^{D P L}-C_{8}$

This peptide was synthesized following general protocol B and purification with achieved using neutral alumina to yield a colorless solid $(0.31 \mathrm{~g}, 54 \%$ overall yield).

${ }^{1} \mathrm{H}-\mathrm{NMR} \quad\left(400 \mathrm{MHz}, \mathrm{CDCl}_{3}\right) \delta=7.91(\mathrm{~d}, J=8.0 \mathrm{~Hz}, 1 \mathrm{H}), 6.37(\mathrm{br}, 1 \mathrm{H}), 4.30(\mathrm{q}, J=8.6$ $\mathrm{Hz}, 1 \mathrm{H}), 3.77(\mathrm{dd}, J=8.4,5.4 \mathrm{~Hz}, 1 \mathrm{H}), 3.28-3.12(\mathrm{~m}, 2 \mathrm{H}), 3.05-2.99(\mathrm{~m}, 1 \mathrm{H}), 2.96-2.91$ (m, 1H), $2.29(\mathrm{br}, 1 \mathrm{H}), 2.19-2.10(\mathrm{~m}, 1 \mathrm{H}), 1.86(\mathrm{sext}, J=6.6 \mathrm{~Hz}, 1 \mathrm{H}), 1.77-1.52(\mathrm{~m}, 5 \mathrm{H})$, $1.45(\mathrm{p}, J=6.6 \mathrm{~Hz}, 2 \mathrm{H}), 1.26(\mathrm{br}, 10 \mathrm{H}), 0.93(\mathrm{~d}, J=6.4 \mathrm{~Hz}, 3 \mathrm{H}), 0.90(\mathrm{~d}, J=6.3 \mathrm{~Hz}, 3 \mathrm{H})$, $0.87(\mathrm{t}, J=6.9 \mathrm{~Hz}, 3 \mathrm{H})$

${ }^{13} \mathrm{C}-\mathrm{NMR}\left(100 \mathrm{MHz}, \mathrm{CDCl}_{3}\right) \delta=175.4,172.1,60.5,51.6,47.4,40.3,39.6,32.0,31.0$, $29.7,29.4(2 \mathrm{C}), 27.1,26.2,25.1,23.1,22.8,22.3,14.3$ 
FT-IR: (KBr pellet) $v=3290(\mathrm{~s}), 3094(\mathrm{w}), 2957(\mathrm{~m}), 2923(\mathrm{~m}), 2853(\mathrm{~m}), 1641(\mathrm{~s}), 1543$ $(\mathrm{m}), 1468(\mathrm{~m}), 1230(\mathrm{~m}), 845(\mathrm{~s}), 721(\mathrm{~m}), 703(\mathrm{~m}) \mathrm{cm}^{-1}$

MP: $72-74^{\circ} \mathrm{C}$

MALDI: expected $\mathrm{m} / \mathrm{z}:[\mathrm{M}+\mathrm{Na}]^{+}, 362.28$ and observed 362.30

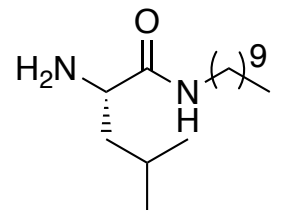

\section{$\mathrm{H}_{2} \mathrm{~N}$-Leu- $\mathrm{C}_{10}$}

This intermediate was synthesized following protocol $A$ to yield a colorless oil $(0.43 \mathrm{~g}, 81 \%$ overall yield).

${ }^{1} \mathrm{H}-\mathrm{NMR} \quad\left(400 \mathrm{MHz}, \mathrm{CDCl}_{3}\right) \delta=7.59(\mathrm{br}, 1 \mathrm{H}), 4.88(\mathrm{brs}, 2 \mathrm{H}), 3.77(\mathrm{br}, 1 \mathrm{H}), 3.33-3.24(\mathrm{~m}$, $1 \mathrm{H}), 3.19-3.11(\mathrm{~m}, 1 \mathrm{H}), 1.72(\mathrm{br}, 2 \mathrm{H}), 1.57-1.49(\mathrm{~m}, 3 \mathrm{H}), 1.31-1.25(\mathrm{~m}, 14 \mathrm{H}), 0.97(\mathrm{~d}, J=$ $6.1 \mathrm{~Hz}, 3 \mathrm{H}), 0.95(\mathrm{~d}, J=6.0 \mathrm{~Hz}, 3 \mathrm{H}), 0.88(\mathrm{t}, J=6.6 \mathrm{~Hz}, 3 \mathrm{H})$

${ }^{13}$ C-NMR $\left(100 \mathrm{MHz}, \mathrm{CDCl}_{3}\right) \delta=175.0,53.5,43.9,39.2,31.9,29.6$ (2C, large peak), 29.4, $27.0,24.9,23.3,22.7,21.6,14.1$

LC-MS (ESI): expected $\mathrm{m} / \mathrm{z}$ : [M + H] $]^{+}, 271.47$ and observed 271.20

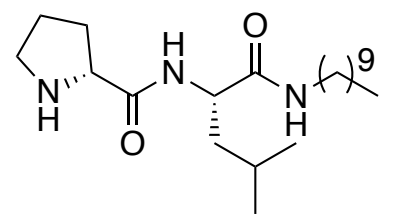

\section{${ }^{D P L}-C_{10}$}

This peptide was synthesized following protocol B to yield a glassy solid $(0.43 \mathrm{~g}, 66 \%$ overall yield).

${ }^{1} \mathrm{H}-\mathrm{NMR} \quad\left(400 \mathrm{MHz}, \mathrm{CDCl}_{3}\right) \delta=7.88(\mathrm{~d}, J=8.3 \mathrm{~Hz}, 1 \mathrm{H}), 6.35(\mathrm{br}, 1 \mathrm{H}), 4.30(\mathrm{td}, J=8.7$, $6.2 \mathrm{~Hz}, 1 \mathrm{H}), 3.74(\mathrm{dd}, J=9.2,5.2 \mathrm{~Hz}, 1 \mathrm{H}), 3.28-3.12(\mathrm{~m}, 2 \mathrm{H}), 3.03-2.97(\mathrm{~m}, 1 \mathrm{H}), 2.93-$ $2.87(\mathrm{~m}, 1 \mathrm{H}), 2.17-2.08(\mathrm{~m}, 1 \mathrm{H}), 1.96(\mathrm{br}, 1 \mathrm{H}), 1.86(\mathrm{sext}, J=6.7 \mathrm{~Hz}, 1 \mathrm{H}), 1.78-1.52(\mathrm{~m}$, $5 \mathrm{H}), 1.45(\mathrm{p}, J=6.7 \mathrm{~Hz}, 2 \mathrm{H}), 1.25(\mathrm{br}, 14 \mathrm{H}), 0.93(\mathrm{~d}, J=6.4 \mathrm{~Hz}, 3 \mathrm{H}), 0.90(\mathrm{~d}, J=6.4 \mathrm{~Hz}$, $3 \mathrm{H}), 0.87(\mathrm{t}, J=7.0 \mathrm{~Hz}, 3 \mathrm{H})$

${ }^{13}$ C-NMR $\left(100 \mathrm{MHz}, \mathrm{CDCl}_{3}\right) \delta=175.9,172.0,60.6,51.4,47.4,40.2,39.6,32.1,31.0$, 29.7 (large peak), 29.5 (2C), 27.0, 26.3, 25.0, 23.1, 22.9, 22.3, 14.3

FT-IR: (KBr pellet) $v$ = $3281(\mathrm{~m}), 3071(\mathrm{w}), 2956(\mathrm{~m}), 2924(\mathrm{~s}), 2853(\mathrm{~m}), 1642(\mathrm{~s}), 1552$ $(\mathrm{m}), 1468(\mathrm{~m}), 1253(\mathrm{~m}), 847(\mathrm{~s}), 711(\mathrm{w}) \mathrm{cm}^{-1}$

MP: $61-63^{\circ} \mathrm{C}$

MALDI: expected $\mathrm{m} / \mathrm{z}:[\mathrm{M}+\mathrm{Na}]^{+}, 390.31$ and observed 390.24 


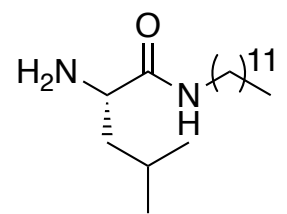

\section{$\mathrm{H}_{2} \mathrm{~N}$-Leu- $\mathrm{C}_{12}$}

This intermediate was synthesized following protocol $A$ to yield a colorless solid $(0.44 \mathrm{~g}, 75 \%$ overall yield).

${ }^{1} \mathrm{H}-\mathrm{NMR} \quad\left(400 \mathrm{MHz}, \mathrm{CDCl}_{3}\right) \delta=7.67(\mathrm{t}, J=5.3 \mathrm{~Hz}, 1 \mathrm{H}), 5.45(\mathrm{br}, 2 \mathrm{H}), 3.40(\mathrm{br}, 1 \mathrm{H}), 3.27-$ $3.19(\mathrm{~m}, 1 \mathrm{H}), 3.14-3.06(\mathrm{~m}, 1 \mathrm{H}), 1.72-1.63(\mathrm{~m}, 2 \mathrm{H}), 1.51-1.43(\mathrm{~m}, 3 \mathrm{H}), 1.24(\mathrm{br}, 18 \mathrm{H})$, $0.92(\mathrm{~d}, J=6.2 \mathrm{~Hz}, 3 \mathrm{H}), 0.90(\mathrm{~d}, J=6.2 \mathrm{~Hz}, 3 \mathrm{H}), 0.87(\mathrm{t}, J=7.0 \mathrm{~Hz}, 3 \mathrm{H})$

${ }^{13} \mathrm{C}-N M R\left(100 \mathrm{MHz}, \mathrm{CDCl}_{3}\right) \delta=175.2,53.7,44.2,39.3,32.1,29.9,29.8(4 \mathrm{C}), 29.5(2 \mathrm{C})$, $27.2,25.1,23.6,22.9,21.6,14.3$

FT-IR: (KBr pellet) v = $3345(\mathrm{~m}), 3309(\mathrm{~m}), 3075(\mathrm{w}), 2956(\mathrm{~m}), 2924(\mathrm{~s}), 2853(\mathrm{~m}), 1649$ $(\mathrm{m}), 1533(\mathrm{~m}), 1468(\mathrm{~m}), 1379(\mathrm{~m}), 845(\mathrm{w}), 721(\mathrm{w}) \mathrm{cm}^{-1}$

\section{MP: $30-33{ }^{\circ} \mathrm{C}$}

LC-MS (ESI): expected $\mathrm{m} / \mathrm{z}$ : [M + H] $]^{+}, 299.31$ and observed 299.25

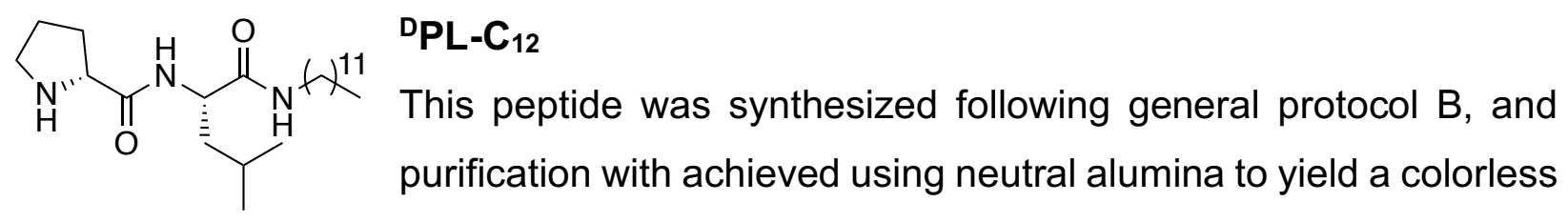
solid $(0.33 \mathrm{~g}, 47 \%$ overall yield).

${ }^{1} \mathrm{H}-\mathrm{NMR} \quad\left(400 \mathrm{MHz}, \mathrm{CDCl}_{3}\right) \delta=7.88(\mathrm{~d}, J=8.4 \mathrm{~Hz}, 1 \mathrm{H}), 6.35(\mathrm{br}, 1 \mathrm{H}), 4.30(\mathrm{td}, J=8.7$, $6.2 \mathrm{~Hz}, 1 \mathrm{H}), 3.75(\mathrm{dd}, J=9.2,5.2 \mathrm{~Hz}, 1 \mathrm{H}), 3.28-3.12(\mathrm{~m}, 2 \mathrm{H}), 3.03-2.97(\mathrm{~m}, 1 \mathrm{H}), 2.93-$ $2.87(\mathrm{~m}, 1 \mathrm{H}), 2.17-2.08(\mathrm{~m}, 1 \mathrm{H}), 1.93-1.80(\mathrm{~m}, 3 \mathrm{H}), 1.78-1.52(\mathrm{~m}, 5 \mathrm{H}), 1.45(\mathrm{p}, J=6.7$ $\mathrm{Hz}, 2 \mathrm{H}), 1.24(\mathrm{br}, 18 \mathrm{H}), 0.93(\mathrm{~d}, J=6.4 \mathrm{~Hz}, 3 \mathrm{H}), 0.90(\mathrm{~d}, J=6.4 \mathrm{~Hz}, 3 \mathrm{H}), 0.87$ (t, J = 7.0 $\mathrm{Hz}, 3 \mathrm{H})$

${ }^{13}$ C-NMR $\left(100 \mathrm{MHz}, \mathrm{CDCl}_{3}\right) \delta=175.9,172.0,60.6,51.4,47.4,40.3,39.6,32.1,31.0$, 29.8 (3C), 29.7 (2C), 29.5 (2C), 27.0, 26.3, 25.0, 23.1, 22.9, 22.3, 14.3

FT-IR: (KBr pellet) v = 3288 (s), 3096 (w), 2957 (m), 2921 (s), 2851 (s), 1641 (s), 1559 $(\mathrm{m}), 1542(\mathrm{~m}), 1468(\mathrm{~m}), 1386(\mathrm{~m}), 1230(\mathrm{~m}), 721(\mathrm{~m}), 700(\mathrm{~m}) \mathrm{cm}^{-1}$

\section{MP: $72-74{ }^{\circ} \mathrm{C}$}

MALDI: expected $\mathrm{m} / \mathrm{z}$ : $[\mathrm{M}+\mathrm{Na}]^{+}, 418.34$ and observed 418.62 


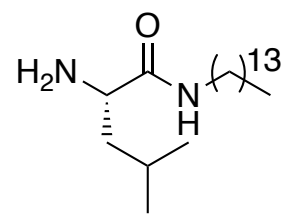

\section{$\mathrm{H}_{2} \mathrm{~N}$-Leu- $\mathrm{C}_{14}$}

This intermediate was synthesized following protocol $A$ to yield a colorless viscous oil $(0.64 \mathrm{~g}, 98 \%$ overall yield).

${ }^{1} \mathrm{H}-\mathrm{NMR}\left(300 \mathrm{MHz}, \mathrm{CDCl}_{3}\right) \delta=7.42(\mathrm{br}, 1 \mathrm{H}), 3.58(\mathrm{br}, 3 \mathrm{H}), 3.28-3.09(\mathrm{~m}, 2 \mathrm{H}), 1.72-1.63$ $(\mathrm{m}, 2 \mathrm{H}), 1.49-1.39(\mathrm{~m}, 3 \mathrm{H}), 1.25(\mathrm{br}, 22 \mathrm{H}), 0.93(\mathrm{~d}, J=7.1 \mathrm{~Hz}, 3 \mathrm{H}), 0.91(\mathrm{~d}, J=6.6 \mathrm{~Hz}$, $3 \mathrm{H}), 0.87(\mathrm{t}, J=6.9 \mathrm{~Hz}, 3 \mathrm{H})$

${ }^{13} \mathrm{C}-\mathrm{NMR}\left(100 \mathrm{MHz}, \mathrm{CDCl}_{3}\right) \delta=173.1,53.2,42.8,39.6,32.1,29.9$ (large peak), $29.8(3 \mathrm{C})$, 29.7, 29.5 (2C), 27.1, 24.8, 23.0, 22.8, 22.0, 14.3

FT-IR: (KBr pellet) v = $3349(\mathrm{~m}), 3296(\mathrm{~m}), 3080(\mathrm{~m}), 2956$ (s), $2922(\mathrm{~s}), 2851(\mathrm{~s}), 1667$ (s), 1624 (s), 1526 (s), 1469 (s), 1202 (s), 1180 (m), 1140 (m), 837 (w), 801 (m), $721(\mathrm{~m})$ $\mathrm{cm}^{-1}$

MP: $35-37^{\circ} \mathrm{C}$

LC-MS (ESI): expected $m / z$ : [M + H] $]^{+}, 327.34$ and observed 327.25

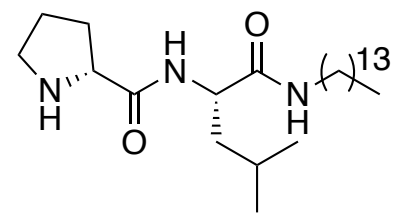

\section{${ }^{\mathrm{PPL}-\mathrm{C}_{14}}$}

This peptide was synthesized following protocol $B$ to yield a colorless solid ( $0.38 \mathrm{~g}, 50 \%$ overall yield).

${ }^{1} \mathrm{H}-\mathrm{NMR} \quad\left(400 \mathrm{MHz}, \mathrm{CDCl}_{3}\right) \delta=8.07(\mathrm{~d}, J=8.4 \mathrm{~Hz}, 1 \mathrm{H}), 6.63(\mathrm{br}, 1 \mathrm{H}), 4.34(\mathrm{td}, J=8.4$, $5.9 \mathrm{~Hz}, 1 \mathrm{H}), 3.90(\mathrm{dd}, J=8.3,5.6 \mathrm{~Hz}, 1 \mathrm{H}), 3.41(\mathrm{br}, 1 \mathrm{H}), 3.23-3.14(\mathrm{~m}, 2 \mathrm{H}), 3.12-2.97(\mathrm{~m}$, 2H), 2.19 (sext, $J=7.6 \mathrm{~Hz}, 1 \mathrm{H}) 1.88$ (sext, $J=6.7 \mathrm{~Hz}, 1 \mathrm{H}), 1.78-1.69(\mathrm{~m}, 3 \mathrm{H}), 1.66-1.52$ $(\mathrm{m}, 2 \mathrm{H}), 1.46(\mathrm{p}, J=6.7 \mathrm{~Hz}, 2 \mathrm{H}), 1.24(\mathrm{br}, 22 \mathrm{H}), 0.93(\mathrm{~d}, J=6.2 \mathrm{~Hz}, 3 \mathrm{H}), 0.90(\mathrm{~d}, J=6.4$ $\mathrm{Hz}, 3 \mathrm{H}), 0.87(\mathrm{t}, J=6.9 \mathrm{~Hz}, 3 \mathrm{H})$

${ }^{13}$ C-NMR $\left(100 \mathrm{MHz}, \mathrm{CDCl}_{3}\right) \delta=174.6,172.1,60.5,51.8,47.3,40.5,39.7,32.1,30.9$, 29.9 (3C, large peak), 29.8 (2C), 29.7, 29.5 (2C), 27.1, 26.0, 25.1, 23.1, 22.9, 22.3, 14.3 FT-IR: (KBr pellet) v = 3289 (s), 3096 (w), 2957 (m), 2921 (s), 2850 (s), 1641 (s), 1560 $(\mathrm{m}), 1542(\mathrm{~m}), 1467(\mathrm{~m}), 1229(\mathrm{w}), 840(\mathrm{w}), 721(\mathrm{w}), 699(\mathrm{w}) \mathrm{cm}^{-1}$

\section{MP: $73-75^{\circ} \mathrm{C}$}

LC-MS (ESI): expected $\mathrm{m} / \mathrm{z}:[\mathrm{M}+\mathrm{H}]^{+} 424.39$ and observed 424.30 


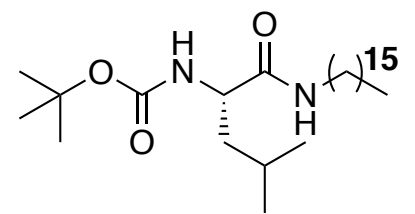

\section{Boc-Leu-C ${ }_{16}$}

To a flame-dried $100 \mathrm{~mL}$ round bottom flask equipped with magnetic stir bar was added Boc-L-Leu-OH $(2.27 \mathrm{~g}, 9.1 \mathrm{mmol})$, HBTU (4.14 g, $10.9 \mathrm{mmol})$, and DMF (40 mL). To the stirred solution was added DIPEA (3.2 $\mathrm{mL}, 18.2 \mathrm{mmol}$ ) via syringe and after complete addition was stirred for ca. 5 mins. Hexadecylamine $(2.64 \mathrm{~g}, 10.9 \mathrm{mmol})$ was then added slowly in one portion to the round bottom flask. The mixture was then allowed to stir at room temperature for one hour. The reaction was stopped and then transferred to a separatory funnel. The round bottom flask was rinsed several times with ethyl acetate and transferred to the separatory funnel ( 300 $\mathrm{mL}$ total). The organics were washed with saturated $\mathrm{NaHCO}_{3}(2 \times 50 \mathrm{~mL})$, then with water $(4 \times 150 \mathrm{~mL})$, and finally with saturated brine ( $\sim 50 \mathrm{~mL})$. The organic layer was dried over $\mathrm{MgSO}_{4}$, filtered over a fritted funnel and concentrated to produce a pale yellow solid. The solid was recrystallized from 1:3 EtOAc:hexanes, filtered over a frit and rinsed with cold hexanes to yield a white powder $(1.35 \mathrm{~g}, 33 \%)$. Note 1: Purification by silica gel chromatography and eluting with EtOAc:hexanes $(1: 9-2: 1)$ will provide higher isolated yields. Note 2: The Boc intermediate and impurities are typically carried through the syntheses until purification of the deprotected dipeptide.

${ }^{1} \mathrm{H}-\mathrm{NMR} \quad\left(400 \mathrm{MHz}, \mathrm{CDCl}_{3}\right) \delta=6.03,5.74(\mathrm{br}, 1 \mathrm{H}), 4.83(\mathrm{br}, 1 \mathrm{H}), 4.03(\mathrm{br}, 1 \mathrm{H}), 3.23(\mathrm{q}$, $J=6.6 \mathrm{~Hz}, 2 \mathrm{H}), 3.00(\mathrm{br}, 1 \mathrm{H}), 1.71-1.60(\mathrm{~m}, 2 \mathrm{H}) 1.50-1.44(\mathrm{~m}, 11 \mathrm{H}), 1.25(\mathrm{br}, 26 \mathrm{H}), 0.94$ $(\mathrm{d}, J=4.2 \mathrm{~Hz}, 3 \mathrm{H}), 0.93(\mathrm{~d}, J=2.8 \mathrm{~Hz}, 3 \mathrm{H}), 0.88(\mathrm{t}, J=6.5 \mathrm{~Hz}, 3 \mathrm{H})$

${ }^{13} \mathrm{C}$-NMR $\left(100 \mathrm{MHz}, \mathrm{CDCl}_{3}\right) \delta=172.6,161.8,156.0,80.1,53.4,45.8,41.5,40.0,39.7$, 32.1, 31.1, 30.0, 29.9 (large peak), 29.8 (2C) 29.7 (2C), 29.5 (2C), 29.3, 28.5, 27.0, 26.9, $24.9,23.1,22.9,22.3,14.3$

FT-IR: (KBr pellet) $v=3330(\mathrm{~m}), 3289(\mathrm{~m}), 2956(\mathrm{~m}), 2918(\mathrm{~s}), 2850(\mathrm{~m}), 1688(\mathrm{~m}), 1657$ (s), $1542(m), 1169(m), 1048(w), 1028(w), 839(w) \mathrm{cm}^{-1}$

MP: $58-60^{\circ} \mathrm{C}$

Note 3: All Boc protected amino acids and Boc-peptide intermediates examined by ${ }^{1} \mathrm{H}$ NMR showed rotamers under ambient temperatures. 


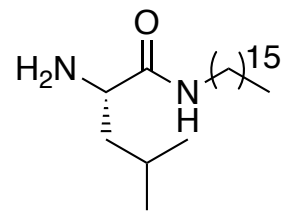

\section{$\mathrm{H}_{2} \mathrm{~N}$-Leu- $\mathrm{C}_{16}$}

This intermediate was synthesized following general protocol A, BocLeu- $\mathrm{C}_{16}(617 \mathrm{mg}, 1.35 \mathrm{mmol})$ and $5 \mathrm{~mL}$ of TFA. Purification afforded an off-white solid ( $460 \mathrm{mg}, 96 \%$ yield).

${ }^{1} \mathrm{H}-\mathrm{NMR} \quad\left(400 \mathrm{MHz}, \mathrm{CDCl}_{3}\right) \delta=7.25(\mathrm{br}, 1 \mathrm{H}), 3.38(\mathrm{dd}, J=10.0,3.5 \mathrm{~Hz}, 1 \mathrm{H}), 3.22(\mathrm{q}, J$ $=6.8 \mathrm{~Hz}) 1.90(\mathrm{br}, 2 \mathrm{H}), 1.75-1.66(\mathrm{~m}, 2 \mathrm{H}), 1.49(\mathrm{p}, J=7.1 \mathrm{~Hz}, 2 \mathrm{H}), 1.36-1.24(\mathrm{~m}, 28 \mathrm{H})$, $0.95(\mathrm{~d}, J=6.2 \mathrm{~Hz}, 3 \mathrm{H}), 0.92(\mathrm{~d}, J=6.1 \mathrm{~Hz}, 3 \mathrm{H}), 0.8 \&(\mathrm{t}, J=7.0 \mathrm{~Hz}, 3 \mathrm{H})$

${ }^{13} \mathrm{C}$-NMR $\left(100 \mathrm{MHz}, \mathrm{CDCl}_{3}\right) \delta=175.4,53.7,44.3,39.3,32.1,29.9$ (2C, large peak), 29.8 (3C), 29.6, 29.5, 27.1, 25.1, 23.6, 22.9, 21.6, 14.3

FT-IR: (KBr pellet) v = $3406(\mathrm{~m}), 3351(\mathrm{~m}), 2958(\mathrm{~m}), 2919$ (s), 2849 (s), 1624 (s), 1526 $(\mathrm{m}), 1470(\mathrm{~m}), 1143(\mathrm{w}), 720(\mathrm{w}) \mathrm{cm}^{-1}$

MP: $53-56{ }^{\circ} \mathrm{C}$

LC-MS (ESI): expected $\mathrm{m} / \mathrm{z}$ : [M + H] $]^{+}, 355.37$ and observed 355.30

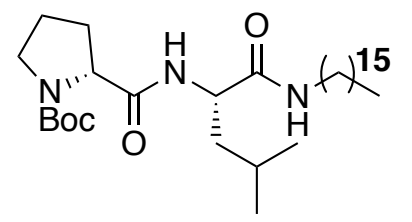

\section{Boc-DPL-C 16}

To a flame-dried $50 \mathrm{~mL}$ round bottom flask equipped with magnetic stir bar $\mathrm{H}_{2} \mathrm{~N}-L e u-\mathrm{C}_{16}(709.2 \mathrm{mg}, 2.0 \mathrm{mmol})$, Boc-D-Pro$\mathrm{OH}$ (478.3 mg, $2.2 \mathrm{mmol}), \mathrm{HBTU}(842.7 \mathrm{mg}, 2.2 \mathrm{mmol}$ ), and DMF $(10 \mathrm{~mL})$ were added. To the stirred solution DIPEA $(0.70 \mathrm{~mL}, 4.0 \mathrm{mmol})$ was added dropwise via syringe. After the addition was complete the mixture was allowed to stir at room temperature for one hour and then transferred to a separatory funnel. The round bottom flask was rinsed several times with EtOAc and transferred to the separatory funnel (200 mL total). The organics were washed with saturated $\mathrm{NaHCO}_{3}(2 \times 50 \mathrm{~mL})$ then water $(4 \times 100 \mathrm{~mL})$, and finally with saturated brine $(50 \mathrm{~mL})$. The organics were dried over $\mathrm{MgSO}_{4}$, filtered over a fritted funnel and concentrated to afford a yellow solid. Purification by silica gel chromatography and eluting with 1:9 to 2:1 gradient of EtOAc:Hexanes results in isolation of a white solid $(1.10 \mathrm{~g}, 83 \%)$.

${ }^{1} \mathrm{H}-\mathrm{NMR} \quad\left(400 \mathrm{MHz}, \mathrm{CDCl}_{3}\right) \delta=6.82,6.21(\mathrm{br}, 1 \mathrm{H}), 6.47$ (brs, $\left.1 \mathrm{H}\right), 4.39(\mathrm{td}, J=9.0,4.9$ $\mathrm{Hz}, 1 \mathrm{H}), 4.15(\mathrm{br}, 1 \mathrm{H}), 3.46,3.37(\mathrm{~m}, 2 \mathrm{H}), 3.19(\mathrm{br}, 2 \mathrm{H}), 2.18-1.91(\mathrm{~m}, 3 \mathrm{H}) 1.84(\mathrm{br}, 2 \mathrm{H})$, 1.63 (sept, $J=6.7 \mathrm{~Hz}, 1 \mathrm{H}), 1.54-1.43(\mathrm{~m}, 12 \mathrm{H}), 1.23(\mathrm{br}, 26 \mathrm{H}), 0.90(\mathrm{t}, J=6.2 \mathrm{~Hz}, 6 \mathrm{H})$, $0.86(\mathrm{t}, J=7.0 \mathrm{~Hz}, 3 \mathrm{H})$ 
${ }^{13}$ C-NMR $\left(100 \mathrm{MHz}, \mathrm{CDCl}_{3}\right) \delta=172.7,171.8,155.6,80.7,61.4,60.8,51.9,47.4,41.3$, 40.6, 39.8, 32.1, 29.9 (large peak), 29.8 (2C), 29.7, 29.5 (2C), 28.5, 27.1, 25.0, 23.2, 22.9, $21.9,14.3$

FT-IR: (KBr pellet) $v=3335(\mathrm{~m}), 3261(\mathrm{~m}), 3050(\mathrm{w}), 2957(\mathrm{~m}), 2924(\mathrm{~m}), 2853(\mathrm{~m}), 1685$ (s), $1639(\mathrm{~s}), 1553(\mathrm{~m}), 1419(\mathrm{~m}), 1164(\mathrm{~m}), 772(\mathrm{w}), 685(\mathrm{w}) \mathrm{cm}^{-1}$

MP: $58-60{ }^{\circ} \mathrm{C}$

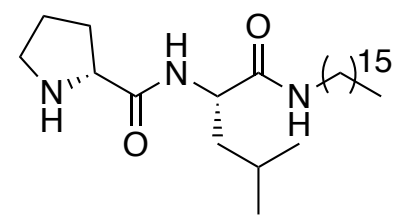

\section{${ }^{D P L}-C_{16}$}

To the flask containing Boc-DPro-Leu- $\mathrm{C}_{16}(6.18 \mathrm{~g}, 11.2 \mathrm{mmol})$ was added a magnetic stirring bar and trifluoroacetic acid (ca. $20 \mathrm{~mL}$ ).

The solution was then stirred for one hour under ambient conditions in air. TFA was then removed by blowing air over it until a viscous oil remained. The excess TFA was then neutralized with saturated $\mathrm{NaHCO}_{3}(\mathrm{pH}=8)$. The solution was then transferred to a separatory funnel. The round bottom flask was then rinsed several times with EtOAc (100 $\mathrm{mL}$ total) and added to the separatory funnel. The organics were separated, and then the organics were extracted from the aqueous layer using EtOAc $(2 \times 75 \mathrm{~mL})$. The combined organics were washed with saturated brine, dried over $\mathrm{MgSO}_{4}$, filtered over a fritted funnel and concentrated to afford a light-yellow solid. $5 \mathrm{~mL}$ of hexanes was added and the solid was heated until completely dissolved. After precipitation, the solid was then triturated in hexanes and filtered over a coarse fritted funnel. The residual solid was washed with cold hexanes and a white powder was isolated $(2.4 \mathrm{~g})$. The mother liquor was concentrated and a second round of trituration was performed in a similar manner, also yielding a white powder ( $1.4 \mathrm{~g}, 3.8 \mathrm{~g}$ total yield, $75 \%$ ). If trituration was not successful, then the standard purification by chromatography was performed.

${ }^{1} \mathrm{H}-\mathrm{NMR} \quad\left(800 \mathrm{MHz}, \mathrm{CDCl}_{3}\right) \delta=7.88(\mathrm{~d}, J=8.4 \mathrm{~Hz}, 1 \mathrm{H}), 6.35(\mathrm{br}, 1 \mathrm{H}), 4.30(\mathrm{td}, \mathrm{J}=8.7$, $6.2 \mathrm{~Hz}, 1 \mathrm{H}), 3.74(\mathrm{dd}, J=9.2,5.3 \mathrm{~Hz}, 1 \mathrm{H}), 3.25-3.21(\mathrm{~m}, 1 \mathrm{H}) 3.18-3.14(\mathrm{~m}, 1 \mathrm{H}), 3.00(\mathrm{dt}$, $J=10.2,6.8 \mathrm{~Hz}, 1 \mathrm{H}), 2.90(\mathrm{dt}, J=10.2,6.3 \mathrm{~Hz} 1 \mathrm{H}), 2.15-2.10(\mathrm{~m}, 1 \mathrm{H}) 1.98(\mathrm{br}, 1 \mathrm{H}), 1.86$ (sext, $J=6.6 \mathrm{~Hz}, 1 \mathrm{H}), 1.76-1.72(\mathrm{~m}, 1 \mathrm{H}), 1.69-1.67(\mathrm{~m}, 2 \mathrm{H}), 1.64-1.60(\mathrm{~m}, 1 \mathrm{H}), 1.57-1.53$ (m, 1H), 1.45 (p, J = 6.9 Hz, 2H), $1.25(\mathrm{br}, 26 \mathrm{H}), 0.93(\mathrm{~d}, J=6.6 \mathrm{~Hz}, 3 \mathrm{H}), 0.90(\mathrm{~d}, J=6.6$ $\mathrm{Hz}, 3 \mathrm{H}), 0.87(\mathrm{t}, J=7.2 \mathrm{~Hz}, 3 \mathrm{H})$ 
${ }^{13} \mathrm{C}$-NMR $\left(100 \mathrm{MHz}, \mathrm{CDCl}_{3}\right) \delta=175.8,172.0,60.6,51.5,47.4,40.2,39.6,32.1,31.0$, 29.9 (3C, large peak), 29.8 (2C), 29.6, 29.5, 27.1, 26.3, 25.1, 23.1, 22.9, 22.3, 14.3

FT-IR: (KBr pellet) v = $3288(\mathrm{~m}), 3095(\mathrm{w}), 2957(\mathrm{~m}), 2920(\mathrm{~s}), 2850(\mathrm{~m}), 1641(\mathrm{~s}), 1542$ $(\mathrm{m}), 1458(\mathrm{~m}), 1384(\mathrm{~m}), 1230(\mathrm{w}), 857(\mathrm{w}), 706(\mathrm{w}) \mathrm{cm}^{-1}$

MP: $58-60^{\circ} \mathrm{C}$

MALDI: expected $\mathrm{m} / \mathrm{z}:[\mathrm{M}+\mathrm{H}]^{+}, 474.40$ and observed 474.39

The 2D COSY NMR spectra for this compound is given in Figure S52.

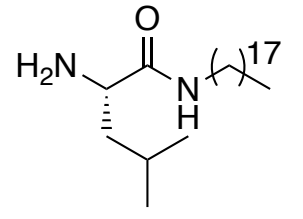

\section{$\mathrm{H}_{2} \mathrm{~N}$-Leu- $\mathrm{C}_{18}$}

This intermediate was synthesized following protocol A to yield a white solid $(0.2 \mathrm{~g}, 24 \%$ overall yield).

${ }^{1} \mathrm{H}-\mathrm{NMR} \quad\left(400 \mathrm{MHz}, \mathrm{CDCl}_{3}\right) \delta=7.29(\mathrm{t}, J=5.6 \mathrm{~Hz}, 1 \mathrm{H}), 3.47(\mathrm{dd}, J=9.4,3.7 \mathrm{~Hz}, 1 \mathrm{H})$, 3.27-3.18 (m, 2H), $2.63(\mathrm{br}, 2 \mathrm{H}), 1.77-1.66(\mathrm{~m}, 2 \mathrm{H}), 1.49(\mathrm{p}, J=7.0 \mathrm{~Hz}, 2 \mathrm{H}), 1.41-1.36$ $(\mathrm{m}, 1 \mathrm{H}), 1.25(\mathrm{br}, 30 \mathrm{H}), 0.96(\mathrm{~d}, J=6.2 \mathrm{~Hz}, 3 \mathrm{H}), 0.93(\mathrm{~d}, J=6.0 \mathrm{~Hz}, 3 \mathrm{H}), 0.88(\mathrm{t}, J=6.5$ $\mathrm{Hz}, 3 \mathrm{H})$

${ }^{13} \mathrm{C}$-NMR $\left(100 \mathrm{MHz}, \mathrm{CDCl}_{3}\right) \delta=174.6,53.6,43.8,39.4,32.1,29.9$ (2C, large peak), 29.8 (3C), 29.6, 29.5, 27.2, 25.1, 23.5, 22.9, 21.7, 14.3

FT-IR: (KBr pellet) $v=3352(\mathrm{~m}), 3307(\mathrm{~m}), 2957(\mathrm{~m}), 2919(\mathrm{~s}), 2849(\mathrm{~s}), 1625(\mathrm{~m}), 1526$ $(\mathrm{m}), 1470(\mathrm{~m}), 1382(\mathrm{w}), 1242(\mathrm{w}), 1142(\mathrm{w}), 720(\mathrm{~m}) \mathrm{cm}^{-1}$

\section{MP: $57-60{ }^{\circ} \mathrm{C}$}

LC-MS (ESI): expected $\mathrm{m} / \mathrm{z}$ : [M + H] $]^{+}, 383.40$ and observed 383.35

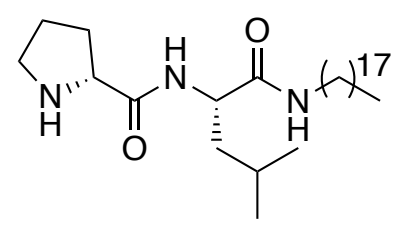

${ }^{D P L-C} 18$

This peptide was synthesized following general protocol $B$, and purification was performed using neutral alumina to yield a white solid $(0.25 \mathrm{~g}, 29 \%$ overall yield).

${ }^{1} \mathrm{H}-\mathrm{NMR} \quad\left(400 \mathrm{MHz}, \mathrm{CDCl}_{3}\right) \delta=8.06(\mathrm{~d}, J=8.4 \mathrm{~Hz}, 1 \mathrm{H}), 6.61(\mathrm{t}, J=5.0 \mathrm{~Hz}, 1 \mathrm{H}), 4.34$ (td, $J=8.5,5.9 \mathrm{~Hz}, 1 \mathrm{H}), 3.89(\mathrm{dd}, J=8.9,5.5 \mathrm{~Hz}, 1 \mathrm{H}), 3.67(\mathrm{br}, 1 \mathrm{H}), 3.22-3.14(\mathrm{~m}, 2 \mathrm{H})$ 3.10-2.96 (m, 2H), 2.23-2.14 (m, 1H), 1.88 (sext, $J=6.5 \mathrm{~Hz}, 1 \mathrm{H}), 1.79-1.68(\mathrm{~m}, 3 \mathrm{H}), 1.66-$ $1.52(\mathrm{~m}, 2 \mathrm{H}), 1.45$ (p, J = 6.5 Hz, 2H), $1.24(\mathrm{br}, 30 \mathrm{H}), 0.92(\mathrm{~d}, J=6.3 \mathrm{~Hz}, 3 \mathrm{H}), 0.89$ (d, J $=6.4 \mathrm{~Hz}, 3 \mathrm{H}), 0.87(\mathrm{t}, J=7.0 \mathrm{~Hz}, 3 \mathrm{H})$ 
${ }^{13}$ C-NMR $\left(100 \mathrm{MHz}, \mathrm{CDCl}_{3}\right) \delta=174.6,172.0,60.5,51.7,47.3,40.5,39.7,32.1,31.0$, 29.9 (2C, large peak), 29.8 (2C), 29.7, 29.6, 29.5, 27.1, 26.0, 25.1, 23.1, 22.9, 22.3, 14.3 FT-IR: (KBr pellet) v = 3290 (s), 3095 (w), 2956 (m), 2919 (s), 2850 (s), 1641 (s), 1559 $(\mathrm{m}), 1542(\mathrm{~m}), 1467(\mathrm{~m}), 1386(\mathrm{~m}), 1229(\mathrm{~m}), 838(\mathrm{~m}), 719(\mathrm{~m}), 702(\mathrm{~m}) \mathrm{cm}^{-1}$

\section{MP: $83-85^{\circ} \mathrm{C}$}

MALDI: expected $\mathrm{m} / \mathrm{z}:[\mathrm{M}+\mathrm{Na}]^{+}, 502.43$ and observed 502.52

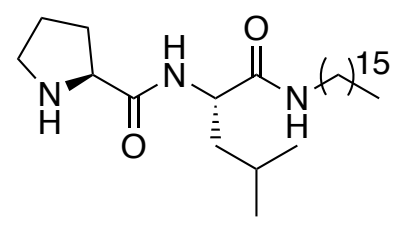

\section{PL-C 16}

This peptide was synthesized following protocol $B$ with minor modifications, $\mathrm{H}_{2} \mathrm{~N}-$ Leu- $\mathrm{C}_{16}$ (460 mg, $1.3 \mathrm{mmol}$ ), HBTU (516.5 mg, $1.36 \mathrm{mmol}$ ), Boc-L-Pro-OH (293.2 mg, $1.36 \mathrm{mmol})$, DMF (10 mL) and DIPEA $(0.45 \mathrm{~mL}, 2.6 \mathrm{mmol})$ were added. Purification using silica gel afforded a paleyellow solid (207 $\mathrm{mg}, 35 \%)$.

${ }^{1} \mathrm{H}-\mathrm{NMR} \quad\left(400 \mathrm{MHz}, \mathrm{CDCl}_{3}\right) \delta=7.96(\mathrm{~d}, J=8.6 \mathrm{~Hz}, 1 \mathrm{H}), 6.48(\mathrm{t}, J=5.4 \mathrm{~Hz}, 1 \mathrm{H}), 4.35-$ $4.29(\mathrm{~m}, 1 \mathrm{H}), 3.72(\mathrm{dd}, J=9.2,5.1 \mathrm{~Hz}, 1 \mathrm{H}), 3.26-3.10(\mathrm{~m}, 2 \mathrm{H}) 3.03-2.98(\mathrm{~m}, 1 \mathrm{H}), 2.91-$ $2.85(\mathrm{~m}, 1 \mathrm{H}), 2.16-2.07(\mathrm{~m}, 2 \mathrm{H}) 1.88(\mathrm{sext}, J=6.6 \mathrm{~Hz}, 1 \mathrm{H}), 1.74-1.64(\mathrm{~m}, 3 \mathrm{H}), 1.62-1.51$ (m, 2H), $1.47(\mathrm{p}, J=6.9 \mathrm{~Hz}, 2 \mathrm{H}), 1.24(\mathrm{br}, 26 \mathrm{H}), 0.92(\mathrm{~d}, J=6.3 \mathrm{~Hz}, 3 \mathrm{H}), 0.89(\mathrm{~d}, J=6.3$ $\mathrm{Hz}, 3 \mathrm{H}), 0.86(\mathrm{t}, \mathrm{J}=7.0 \mathrm{~Hz}, 3 \mathrm{H})$

${ }^{13} \mathrm{C}-N M R\left(100 \mathrm{MHz}, \mathrm{CDCl}_{3}\right) \delta=175.7,172.0,60.6,51.4,47.5,40.6,39.7,32.1,31.0$, 29.9 (large peak), 29.8 (2C), 29.7, 29.6, 29.5 (2C), 27.1, 26.4, 25.1, 23.1, 22.9, 22.2, 14.3 FT-IR: (KBr pellet) v = $3297(\mathrm{~m}), 3097(\mathrm{w}), 2956(\mathrm{~m}), 2918(\mathrm{~s}), 2850(\mathrm{~m}), 1641(\mathrm{~s}), 1554$ $(\mathrm{m}), 1538(\mathrm{~m}), 1466(\mathrm{~m}), 1226(\mathrm{~m}), 721(\mathrm{~m}) \mathrm{cm}^{-1}$

\section{MP: $67-69^{\circ} \mathrm{C}$}

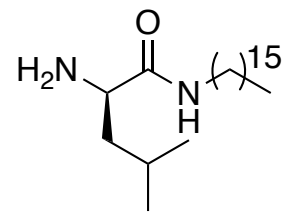

\section{$\mathrm{H}_{2} \mathrm{~N}-{ }^{-}$Leu- $\mathrm{C}_{16}$}

Following general protocol A, Boc-L-Leucine $(1.0 \mathrm{mmol}, 252 \mathrm{mg})$, hexadecylamine (1.1 mmol, $280 \mathrm{mg})$, HBTU (1.1 mmol, $421.4 \mathrm{mg})$ were dissolved in $5 \mathrm{~mL}$ of DMF followed by addition of DIPEA $(2.0 \mathrm{mmol}, 0.35 \mathrm{~mL})$. Purification using silica gel afforded a white solid (324 mg, $91 \%$ overall yield). 
${ }^{1} \mathrm{H}-\mathrm{NMR} \quad\left(400 \mathrm{MHz}, \mathrm{CDCl}_{3}\right) \delta=7.32(\mathrm{br}, 1 \mathrm{H}), 3.44(\mathrm{br}, 1 \mathrm{H}), 3.25-3.17(\mathrm{~m}, 2 \mathrm{H}), 2.53(\mathrm{br}$, 2H), 1.76-1.66 (m, 2H) $1.48(\mathrm{p}, J=6.9 \mathrm{~Hz}, 3 \mathrm{H}), 1.41-1.34(\mathrm{~m}, 1 \mathrm{H}), 1.24$ (br, 26H), 0.95 (d, $J=6.2 \mathrm{~Hz}, 3 \mathrm{H}), 0.92(\mathrm{~d}, J=6.0 \mathrm{~Hz}, 3 \mathrm{H}), 0.87(\mathrm{t}, J=7.0 \mathrm{~Hz}, 3 \mathrm{H})$

${ }^{13} \mathrm{C}-\mathrm{NMR}\left(100 \mathrm{MHz}, \mathrm{CDCl}_{3}\right) \delta=174.9,53.6,43.9,39.4,32.1,29.9$ (2C, large peak), 29.8 (2C), 29.6, 29.5, 27.1, 25.0, 23.5, 22.9, 21.7, 14.3

FT-IR: (KBr pellet) $v=3352(\mathrm{~m}), 2957(\mathrm{~m}), 2922(\mathrm{~s}), 2851(\mathrm{~m}), 1648(\mathrm{~s}), 1526(\mathrm{~m}), 1469$ $(\mathrm{m}), 1203(\mathrm{w}), 1141(\mathrm{w}), 720(\mathrm{w}) \mathrm{cm}^{-1}$

\section{MP: $49-51^{\circ} \mathrm{C}$}

LC-MS (ESI): expected $\mathrm{m} / \mathrm{z}$ : [M + H] $]^{+}, 355.37$ and observed 355.80

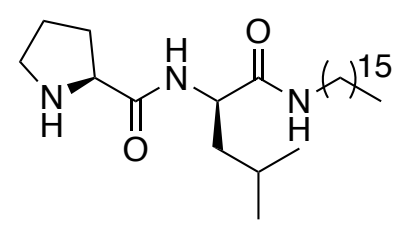

\section{$\mathrm{P}^{\mathrm{D} L-\mathrm{C}_{16}}$}

Following general protocol $B$ with minor modifications, $\mathrm{H}_{2} \mathrm{~N}-\mathrm{D}$ Leu$\mathrm{C}_{16}(177.3 \mathrm{mg}, 0.5 \mathrm{mmol})$, HBTU $(210.7 \mathrm{mg}, 0.55 \mathrm{mmol})$, and BocL-Pro-OH (119.6 mg, $0.55 \mathrm{mmol})$, were dissolved in DMF $(5 \mathrm{~mL})$ followed by addition of DIPEA (0.17 mL, $1.0 \mathrm{mmol})$. Purification using silica gel yielded a white solid (175 mg, $77 \%$ overall yield).

${ }^{1} \mathrm{H}-\mathrm{NMR} \quad\left(400 \mathrm{MHz}, \mathrm{CDCl}_{3}\right) \delta=7.95(\mathrm{~d}, J=8.4 \mathrm{~Hz}, 1 \mathrm{H}), 6.50(\mathrm{t}, J=5.2 \mathrm{~Hz}, 1 \mathrm{H}), 4.32$ (td, $J=8.7,6.2 \mathrm{~Hz}, 1 \mathrm{H}), 3.77(\mathrm{dd}, J=9.0,5.3 \mathrm{~Hz}, 1 \mathrm{H}), 3.25-3.11(\mathrm{~m}, 2 \mathrm{H}) 3.04-2.98(\mathrm{~m}$, $1 \mathrm{H}), 2.95-2.89(\mathrm{~m}, 1 \mathrm{H}), 2.86(\mathrm{br}, 1 \mathrm{H}), 2.18-2.09(\mathrm{~m}, 1 \mathrm{H}) 1.86$ (sext, $J=6.8 \mathrm{~Hz}, 1 \mathrm{H}), 1.76-$ $1.50(\mathrm{~m}, 5 \mathrm{H}), 1.44(\mathrm{t}, J=6.7 \mathrm{~Hz}, 2 \mathrm{H}), 1.24(\mathrm{br}, 26 \mathrm{H}), 0.92(\mathrm{~d}, J=6.4 \mathrm{~Hz}, 3 \mathrm{H}), 0.89(\mathrm{~d}, J$ $=6.4 \mathrm{~Hz}, 3 \mathrm{H}), 0.86(\mathrm{t}, J=7.0 \mathrm{~Hz}, 3 \mathrm{H})$

${ }^{13} \mathrm{C}-N M R\left(100 \mathrm{MHz}, \mathrm{CDCl}_{3}\right) \delta=175.5,172.0,60.5,51.5,47.4,40.3,39.6,32.1,31.0$, 29.9 (2C, large peak), 29.8 (2C), 29.7 (2C), 29.5 (2C), 27.1, 26.2, 25.1, 23.1, 22.9, 22.3, 14.3

FT-IR: (KBr pellet) v = 3289 (s), 3095 (w), 2957 (m), 2920 (s), 2850 (s), 1641 (s), 1560 (m), $1542(\mathrm{~m}), 1467(\mathrm{~m}), 1386(\mathrm{~m}), 1229(\mathrm{~m}), 720(\mathrm{~m}), 699(\mathrm{~m}) \mathrm{cm}^{-1}$

\section{MP: $80-82{ }^{\circ} \mathrm{C}$}

LC-MS (ESI): expected $\mathrm{m} / \mathrm{z}$ : [M + H] $]^{+}, 452.42$ and observed 452.30 


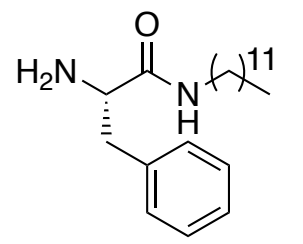

\section{$\mathrm{H}_{2} \mathrm{~N}-\mathrm{Phe}-\mathrm{C}_{12}$}

This intermediate was synthesized similarly to protocol A using Boc-Lphenylalanine $(6.0 \mathrm{mmol}, 1.59 \mathrm{~g})$, dodecylamine $(5.0 \mathrm{mmol}, 0.92 \mathrm{~g})$, and HCTU $(6.0 \mathrm{mmol}, 2.48 \mathrm{~g})$ in $25 \mathrm{~mL}$ of DMF and DIPEA $(8.0 \mathrm{mmol}, 1.34$ $\mathrm{mL})$. Purification yielded a white solid (1.28 g, $65 \%$ overall yield).

${ }^{1} \mathrm{H}-\mathrm{NMR} \quad\left(400 \mathrm{MHz}, \mathrm{CDCl}_{3}\right) \delta=7.31(\mathrm{t}, J=6.9 \mathrm{~Hz}, 2 \mathrm{H}), 7.24-7.21(\mathrm{~m}, 4 \mathrm{H}), 3.60(\mathrm{dd}, J=$ 8.8, 3.4 Hz, 1H), 3.29-3.21 (m, 3H), 2.69 (dd, J = 13.7, $9.3 \mathrm{~Hz}, 1 \mathrm{H}), 1.47(\mathrm{p}, J=6.8 \mathrm{~Hz}$, $2 \mathrm{H}), 1.35$ (br, 2H), $1.26(\mathrm{br}, 18 \mathrm{H}), 0.88(\mathrm{t}, J=6.6 \mathrm{~Hz}, 3 \mathrm{H})$

${ }^{13} \mathrm{C}-\mathrm{NMR}\left(100 \mathrm{MHz}, \mathrm{CDCl}_{3}\right) \delta=174.2,138.2,129.5,128.8,126.9,56.7,41.3,39.2,32.1$, 29.8 (3C, large peak), 29.7, 29.5 (2C), 27.1, 22.9, 14.3

FT-IR: (neat) v = $3286(\mathrm{~m}), 2955(\mathrm{~m}), 2916$ (s), $2849(\mathrm{~s}), 1631(\mathrm{~s}), 1549(\mathrm{~m}), 1523(\mathrm{~m})$, $848(\mathrm{~m}), 741(\mathrm{~s}), 718(\mathrm{~s}) \mathrm{cm}^{-1}$

\section{MP: $56-58{ }^{\circ} \mathrm{C}$}

LC-MS (ESI): expected $\mathrm{m} / \mathrm{z}$ : [M + H] $]^{+}, 333.29$ and observed 333.25

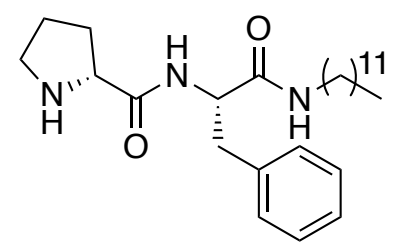

\section{${ }^{D P F}-C_{12}$}

This peptide was synthesized following protocol $B$ to yield a colorless solid ( $0.33 \mathrm{~g}, 47 \%$ overall yield).

${ }^{1} \mathrm{H}-\mathrm{NMR} \quad\left(400 \mathrm{MHz}, \mathrm{CDCl}_{3}\right) \delta=8.14(\mathrm{~d}, J=8.2 \mathrm{~Hz}, 1 \mathrm{H}), 7.29-7.19$ (m, 5H), $6.21(\mathrm{br}, 1 \mathrm{H}), 4.52(\mathrm{q}, J=7.6,1 \mathrm{H}), 3.72(\mathrm{dd}, J=9.1,5.3 \mathrm{~Hz}, 1 \mathrm{H}), 3.16-3.10(\mathrm{~m}$, $3 \mathrm{H}), 3.06-2.86(\mathrm{~m}, 3 \mathrm{H}), 2.15-2.05(\mathrm{~m}, 1 \mathrm{H}), 1.81$ (sext, $J=5.9 \mathrm{~Hz}, 1 \mathrm{H}), 1.73-1.59(\mathrm{~m}, 2 \mathrm{H})$, $1.38-1.18(\mathrm{~m}, 21 \mathrm{H}), 0.88(\mathrm{t}, J=6.6 \mathrm{~Hz}, 3 \mathrm{H})$

${ }^{13} \mathrm{C}$-NMR $\left(100 \mathrm{MHz}, \mathrm{CDCl}_{3}\right) \delta=175.3,171.0,137.4,129.5,128.7,127.0,60.5,54.8,47.3$, 39.6, 38.0, 32.1, 30.9, 29.8 (3C), 29.7, 29.5 (3C), 27.0, 26.1, 22.9, 14.3

FT-IR: (neat) v = $3279(\mathrm{~m}), 3088(\mathrm{w}), 2919(\mathrm{~m}), 2850(\mathrm{~m}), 1642(\mathrm{~s}), 1540(\mathrm{~m}), 1380(\mathrm{w})$, $1230(\mathrm{~m}), 915(\mathrm{~m}), 699(\mathrm{~s}) \mathrm{cm}^{-1}$

\section{MP: $105-107^{\circ} \mathrm{C}$}

LC-MS (ESI): expected $\mathrm{m} / \mathrm{z}$ : [M + H] $]^{+}, 430.34$ and observed 430.30 


\section{Characterization Of Transfer Hydrogenation Products}

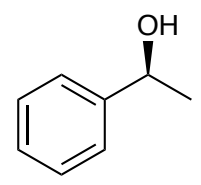

\section{1-phenylethanol ${ }^{5}$}

Following ATH Method 1 or 2, $0.8 \mathrm{~mL}$ of a $3.125 \mathrm{M}$ sodium formate solution in water and acetophenone $(59.5 \mu \mathrm{L}, 0.5 \mathrm{mmol})$ were added to the microtube via micropipette. After 3 hours, the biphasic mixture is then passed through a short silica plug and eluted with MTBE. The solvent was then removed by rotavap to give a colorless to pale-yellow oil and the yield of the reaction was determined by ${ }^{1} \mathrm{H}$ NMR $(76 \%$ conversion), ee $=93 \%(S)$ by HPLC (Lux Cellulose-1, EtOH:Hexanes $=5: 95,1.0 \mathrm{~mL} / \mathrm{min}$ flow rate, $\lambda=254 \mathrm{~nm}$, retention $(\mathrm{min}):(\mathrm{R})=7.9,(\mathrm{~S})=9.8)$.

${ }^{1} \mathrm{H}-\mathrm{NMR} \quad\left(400 \mathrm{MHz}, \mathrm{CDCl}_{3}\right) \delta=7.40-7.34(\mathrm{~m}, 4 \mathrm{H}), 7.30-7.26(\mathrm{~m}, 1 \mathrm{H}), 4.90(\mathrm{q}, J=6.5$ $\mathrm{Hz}, 1 \mathrm{H}), 1.82(\mathrm{brs}, 1 \mathrm{H}), 1.51(\mathrm{~d}, J=6.5 \mathrm{~Hz}, 3 \mathrm{H})$

${ }^{13} \mathrm{C}$-NMR $\left(100 \mathrm{MHz}, \mathrm{CDCl}_{3}\right) \delta=146.0,128.7,127.7,125.6,70.6,25.4$

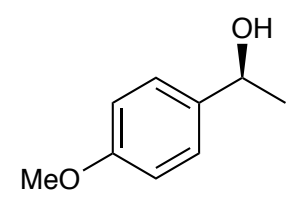

\section{1-(4-methoxyphenyl)ethanol ${ }^{5}$}

Following ATH Method 1 or $2,0.8 \mathrm{~mL}$ of a $3.125 \mathrm{M}$ sodium formate solution in water and 4'-methoxyacetophenone $(75.8 \mathrm{mg}, 0.5 \mathrm{mmol})$ were added to the microtube via micropipette. After 3 hours, the biphasic mixture is then passed through a short silica plug and eluted with MTBE. The solvent was then removed by rotavap to give a pale-yellow oil and the yield of the reaction was determined by ${ }^{1} \mathrm{H}$ NMR (35\% conversion), ee $=91 \%(S)$ by HPLC (Lux Cellulose-1, EtOH:Hexanes $=5: 95$, $1.0 \mathrm{~mL} / \mathrm{min}$ flow rate, $\lambda=254 \mathrm{~nm}$, retention $(\mathrm{min}):(\mathrm{R})=10.3,(\mathrm{~S})=11.5)$.

${ }^{1} \mathrm{H}-\mathrm{NMR} \quad\left(400 \mathrm{MHz}, \mathrm{CDCl}_{3}\right) \delta=7.30(\mathrm{dt}, J=8.6,2.9 \mathrm{~Hz}, 2 \mathrm{H}), 6.89(\mathrm{dt}, J=8.8,2.9 \mathrm{~Hz}$, 2H), 4.85 (q, J = 6.4 Hz, 1H), $3.80(\mathrm{~s}, 3 \mathrm{H}), 1.79$ (brs, 1H), 1.48 (d, J = $6.4 \mathrm{~Hz}, 3 \mathrm{H}$ )

${ }^{13} \mathrm{C}-\mathrm{NMR}\left(100 \mathrm{MHz}, \mathrm{CDCl}_{3}\right) \delta=159.2,138.2,126.9,114.1,70.2,55.5,25.2$

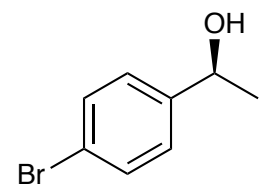

\section{1-(4-bromophenyl)ethanol ${ }^{5}$}

Following ATH Method 1 or $2,0.8 \mathrm{~mL}$ of a $3.125 \mathrm{M}$ sodium formate solution in water and 4'-bromoacetophenone $(100.5 \mathrm{mg}, 0.5 \mathrm{mmol})$ were added to the microtube via micropipette. After 3 hours, the biphasic mixture is then passed through a short silica plug and eluted with MTBE. The solvent was then removed by 
rotavap to give a colorless to pale-yellow oil and the yield of the reaction was determined by ${ }^{1} \mathrm{H}$ NMR (88\% conversion), ee $=90 \%(S)$ by HPLC (Lux Cellulose-1, EtOH:Hexanes $=5: 95,1.0 \mathrm{~mL} / \mathrm{min}$ flow rate, $\lambda=254 \mathrm{~nm}$, retention $(\mathrm{min}):(\mathrm{R})=8.9,(\mathrm{~S})=8.3)$.

${ }^{1} \mathrm{H}-\mathrm{NMR} \quad\left(400 \mathrm{MHz}, \mathrm{CDCl}_{3}\right) \delta=7.46(\mathrm{dt}, J=8.4,2.4 \mathrm{~Hz}, 2 \mathrm{H}), 7.23(\mathrm{dt}, J=8.4,2.3 \mathrm{~Hz}$, $2 \mathrm{H}$ ), $4.84(\mathrm{q}, J=6.4 \mathrm{~Hz}, 1 \mathrm{H}), 2.03(\mathrm{brs}, 1 \mathrm{H}), 1.45(\mathrm{~d}, J=6.5 \mathrm{~Hz}, 3 \mathrm{H})$

${ }^{13} \mathrm{C}$-NMR $\left(100 \mathrm{MHz}, \mathrm{CDCl}_{3}\right) \delta=145.0,131.7,127.3,121.3,69.9,25.4$

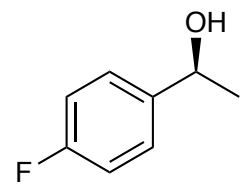

\section{1-(4-fluorophenyl)ethanol ${ }^{5}$}

Following ATH Method 1 or $2,0.8 \mathrm{~mL}$ of a $3.125 \mathrm{M}$ sodium formate solution in water and 4'-fluoroacetophenone $(61.1 \mu \mathrm{L}, 0.5 \mathrm{mmol})$ were added to the microtube via micropipette. After 3 hours, the biphasic mixture is then passed through a short silica plug and eluted with MTBE. The solvent was then removed by rotavap to give a colorless to pale-yellow oil and the yield of the reaction was determined by ${ }^{1} \mathrm{H}$ NMR (77\% conversion), ee $=92 \%(S)$ by GC: (Agilent J\&W Cyclodex-B $(30 \mathrm{~m} \times 0.25 \mathrm{~mm} \times$ $0.25 \mu \mathrm{m}), \mathrm{T}=135^{\circ} \mathrm{C}, \mathrm{P}=1.1 \mathrm{~mL} / \mathrm{min}(8.07 \mathrm{psi}) \mathrm{He}$, detector $=\mathrm{FID} 230^{\circ} \mathrm{C}$, injection $=$ $230^{\circ} \mathrm{C}$, retention $\left.(\min ):(R)=14.8,(S)=15.3\right)$.

${ }^{1} \mathrm{H}-\mathrm{NMR} \quad\left(400 \mathrm{MHz}, \mathrm{CDCl}_{3}\right) \delta=7.34-7.31(\mathrm{~m}, 2 \mathrm{H}), 7.04-7.00(\mathrm{~m}, 2 \mathrm{H}), 4.87(\mathrm{dq}, J=6.4$, $3.4 \mathrm{~Hz}, 1 \mathrm{H}), 2.06(\mathrm{~d}, J=3.4 \mathrm{~Hz}, 1 \mathrm{H}), 1.46(\mathrm{~d}, J=6.4 \mathrm{~Hz}, 3 \mathrm{H})$

${ }^{13} \mathrm{C}$-NMR $\left(100 \mathrm{MHz}, \mathrm{CDCl}_{3}\right) \delta=162.3(\mathrm{~d}, J=243.7 \mathrm{~Hz}), 141.7(\mathrm{~d}, J=3.2 \mathrm{~Hz}), 127.2(\mathrm{~d}$, $J=8.0 \mathrm{~Hz}), 115.4(\mathrm{~d}, J=21.2 \mathrm{~Hz}), 69.9,25.5$

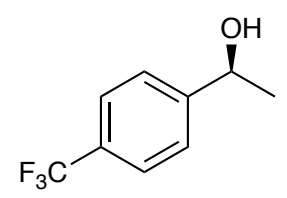

\section{1-[(4-trifluoromethyl)phenyl]ethanol ${ }^{8}$}

Following ATH Method 1 or $2,0.8 \mathrm{~mL}$ of a $3.125 \mathrm{M}$ sodium formate solution in water and 4'-trifluoromethylacetophenone $(96.0 \mathrm{mg}, 0.5$ $\mathrm{mmol}$ ) were added to the microtube via micropipette. After 3 hours, the biphasic mixture is then passed through a short silica plug and eluted with MTBE. The solvent was then removed by rotavap to give a white solid and the yield of the reaction was determined by ${ }^{1} \mathrm{H}$ NMR (99\% conversion, $92 \mathrm{mg}$, (97\% isolated yield)), ee $=93 \%(S)$ by GC: (Agilent J\&W Cyclodex-B $(30 \mathrm{~m} \times 0.25 \mathrm{~mm} \times 0.25 \mu \mathrm{m}), \mathrm{T}=135^{\circ} \mathrm{C}, \mathrm{P}=1.1 \mathrm{~mL} / \mathrm{min}(8.07 \mathrm{psi}) \mathrm{He}$, detector $=\mathrm{FID} 230{ }^{\circ} \mathrm{C}$, injection $=230{ }^{\circ} \mathrm{C}$, retention $\left.(\min ):(R)=18.1,(S)=19.0\right)$. 
${ }^{1} \mathrm{H}-\mathrm{NMR} \quad\left(300 \mathrm{MHz}, \mathrm{CDCl}_{3}\right) \delta=7.60(\mathrm{~d}, J=8.2 \mathrm{~Hz}, 2 \mathrm{H}), 7.48(\mathrm{~d}, J=8.1 \mathrm{~Hz}, 2 \mathrm{H}), 4.95$ (dq, $J=6.5,3.8 \mathrm{~Hz}, 1 \mathrm{H}), 2.10(\mathrm{~d}, J=3.6 \mathrm{~Hz}, 1 \mathrm{H}), 1.49(\mathrm{~d}, J=6.5 \mathrm{~Hz}, 3 \mathrm{H})$

${ }^{13} \mathrm{C}-\mathrm{NMR}\left(100 \mathrm{MHz}, \mathrm{CDCl}_{3}\right) \delta=149.9(\mathrm{~d}, J=1.1 \mathrm{~Hz}), 129.8(\mathrm{q}, J=32.2 \mathrm{~Hz}), 125.9,125.6$ (q, $J=3.7 \mathrm{~Hz}), 124.4$ (q, $J=270.4 \mathrm{~Hz}), 70.0,25.6$

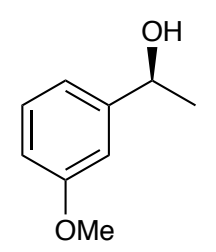

\section{1-(3-methoxyphenyl)ethanol ${ }^{8}$}

Following ATH Method 1 or $2,0.8 \mathrm{~mL}$ of a $3.125 \mathrm{M}$ sodium formate solution in water and 3'-methoxyacetophenone $(70.8 \mu \mathrm{L}, 0.5 \mathrm{mmol})$ were added to the microtube via micropipette. After 3 hours, the biphasic mixture is then passed through a short silica plug and eluted with MTBE. The solvent was then removed by rotavap to give a white solid and the yield of the reaction was determined by ${ }^{1} \mathrm{H}$ NMR (92\% conversion), ee $=91 \%(S)$ by HPLC (Lux Cellulose-1, EtOH:Hexanes $=5: 95,1.0$ $\mathrm{mL} / \mathrm{min}$ flow rate, $\lambda=254 \mathrm{~nm}$, retention $(\min ):(R)=10.9,(S)=14.0)$.

${ }^{1} \mathrm{H}-\mathrm{NMR} \quad\left(400 \mathrm{MHz}, \mathrm{CDCl}_{3}\right) \delta=7.29-7.25(\mathrm{~m}, 1 \mathrm{H}), 6.95(\mathrm{dd}, J=4.0,1.7 \mathrm{~Hz}, 2 \mathrm{H}), 6.82$ (ddd, $J=8.2,2.5,0.8 \mathrm{~Hz}, 2 \mathrm{H}$ ), 4.88 (q, $J=6.4 \mathrm{~Hz}, 1 \mathrm{H}$ ), 3.82 (s, 3H), 1.81 (brs, 1H), 1.50 $(\mathrm{d}, J=6.4 \mathrm{~Hz}, 3 \mathrm{H})$

${ }^{13} \mathrm{C}$-NMR $\left(100 \mathrm{MHz}, \mathrm{CDCl}_{3}\right) \delta=160.0,147.8,129.8,117.9,113.1,111.1,70.6,55.4,25.4$

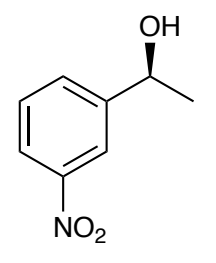

\section{1-(3-nitrophenyl)ethanol ${ }^{9}$}

Following ATH Method 1 or $2,0.8 \mathrm{~mL}$ of a $3.125 \mathrm{M}$ sodium formate solution in water and 3'-nitroacetophenone $(85.1 \mathrm{mg}, 0.5 \mathrm{mmol})$ were added to the microtube via micropipette. After 3 hours, the biphasic mixture is then passed through a short silica plug and eluted with MTBE. The solvent was then removed by rotavap to give a light yellow solid and the yield of the reaction was determined by ${ }^{1} \mathrm{H}$ NMR ( $98 \%$ conversion, $76 \mathrm{mg}(91 \%$ isolated yield)), ee $=83 \%(S)$ by HPLC (Lux Cellulose-1, EtOH:Hexanes = 2:98, $1.0 \mathrm{~mL} / \mathrm{min}$ flow rate, $\lambda=254 \mathrm{~nm}$, retention $(\mathrm{min}):(\mathrm{R})$ $=28.5,(\mathrm{~S})=29.9)$.

${ }^{1} \mathrm{H}-\mathrm{NMR} \quad\left(400 \mathrm{MHz}, \mathrm{CDCl}_{3}\right) \delta=8.25(\mathrm{t}, J=1.6 \mathrm{~Hz}, 1 \mathrm{H}), 8.13(\mathrm{ddd}, J=8.1,2.0,0.8 \mathrm{~Hz}$, 2H), 7.73-7.71 (m, 1H), $7.52(\mathrm{t}, J=8.0 \mathrm{~Hz}, 1 \mathrm{H}), 5.02(\mathrm{q}, J=6.4 \mathrm{~Hz}, 1 \mathrm{H}), 2.03$ (brs, $1 \mathrm{H}$ ), $1.54(\mathrm{~d}, J=6.4 \mathrm{~Hz}, 3 \mathrm{H})$

${ }^{13} \mathrm{C}$-NMR $\left(100 \mathrm{MHz}, \mathrm{CDCl}_{3}\right) \delta=148.6,148.1,131.8,129.7,122.6,120.7,69.6,25.7$ 


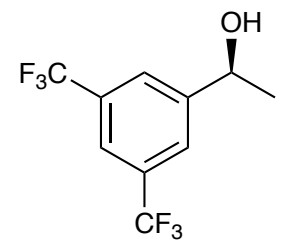

1-[(3,5-bistrifluoromethyl)phenyl]ethanol ${ }^{5}$

Following ATH Method 1 or 2, $0.8 \mathrm{~mL}$ of a $3.125 \mathrm{M}$ sodium formate solution in water and 3',5'-bis(trifluoromethyl)acetophenone $(91.9 \mu \mathrm{L}, 0.5$ $\mathrm{mmol}$ ) were added to the microtube via micropipette. After 3 hours, the biphasic mixture is then passed through a short silica plug and eluted with MTBE. The solvent was then removed by rotavap to give a white solid and the yield of the reaction was determined by ${ }^{1} \mathrm{H}$ NMR (97\% conversion), ee $=94 \%(S)$ by GC: (Agilent $\mathrm{J} \& \mathrm{~W}$ Cyclodex-B (30 m x $0.25 \mathrm{~mm} \times 0.25 \mu \mathrm{m}), \mathrm{T}=135^{\circ} \mathrm{C}, \mathrm{P}=1.1 \mathrm{~mL} / \mathrm{min}(8.07 \mathrm{psi}) \mathrm{He}$, detector $=\mathrm{FID} 230^{\circ} \mathrm{C}$, injection $=230^{\circ} \mathrm{C}$, retention $\left.(\min ):(R)=13.4,(S)=12.9\right)$.

${ }^{1} \mathrm{H}-\mathrm{NMR} \quad\left(300 \mathrm{MHz}, \mathrm{CDCl}_{3}\right) \delta=7.84(\mathrm{~s}, 2 \mathrm{H}) 7.79(\mathrm{~s}, 1 \mathrm{H}), 5.04(\mathrm{q}, J=6.4 \mathrm{~Hz}, 1 \mathrm{H}), 2.10$ (s, $1 \mathrm{H}), 1.54(\mathrm{~d}, J=6.5 \mathrm{~Hz}, 3 \mathrm{H})$

${ }^{13} \mathrm{C}$-NMR $\left(100 \mathrm{MHz}, \mathrm{CDCl}_{3}\right) \delta=148.4,132.0$ (q, $\left.J=33.2 \mathrm{~Hz}\right), 125.9$ (d, $\left.J=2.5 \mathrm{~Hz}\right), 123.6$ (q, $J=271.1 \mathrm{~Hz}) 121.5$ (sept, $J=3.6 \mathrm{~Hz}$ ), 69.5, 25.8

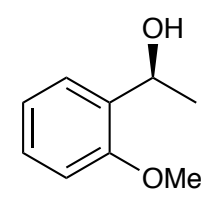

\section{1-(2-methoxyphenyl)ethanol ${ }^{6}$}

Following ATH Method 1 or $2,0.8 \mathrm{~mL}$ of a $3.125 \mathrm{M}$ sodium formate solution in water and 2'-methoxyacetophenone $(68.9 \mu \mathrm{L}, 0.5 \mathrm{mmol})$ were added to the microtube via micropipette. After 3 hours, the biphasic mixture is then passed through a short silica plug and eluted with MTBE. The solvent was then removed by rotavap to give a colorless to pale-yellow oil and the yield of the reaction was determined by ${ }^{1} \mathrm{H}$ NMR (68\% conversion), ee $=73 \%(S)$ by HPLC (Lux Cellulose-1, EtOH:Hexanes $=5: 95,1.0$ $\mathrm{mL} / \mathrm{min}$ flow rate, $\lambda=254 \mathrm{~nm}$, retention $(\min ):(R)=9.5,(S)=8.8)$.

${ }^{1} \mathrm{H}-\mathrm{NMR} \quad\left(400 \mathrm{MHz}, \mathrm{CDCl}_{3}\right) \delta=7.34(\mathrm{dd}, J=7.5,1.6 \mathrm{~Hz}, 1 \mathrm{H}), 7.25(\mathrm{td}, J=8.0,1.8 \mathrm{~Hz}$, 1H), 6.97 (td, $J=7.5,1.0 \mathrm{~Hz}, 1 \mathrm{H}), 6.89(\mathrm{~d}, J=8.2 \mathrm{~Hz}, 1 \mathrm{H}), 5.10$ (q, J = 6.5 Hz, 1H), 3.87 (s, 3H), 2.62 (brs, 1H), 1.51 (d, $J=6.5 \mathrm{~Hz}, 3 \mathrm{H})$

${ }^{13} \mathrm{C}-\mathrm{NMR}\left(100 \mathrm{MHz}, \mathrm{CDCl}_{3}\right) \delta=156.8,133.6,128.5,126.3,121.0,110.6,66.8,55.5,23.0$<smiles>CC(O)c1ccccc1O</smiles>
1-(2-hydroxyphenyl)ethanol ${ }^{10}$

Following ATH Method 1 or $2,0.8 \mathrm{~mL}$ of a $3.125 \mathrm{M}$ sodium formate solution in water and 2'-hydroxyacetophenone $(61.5 \mu \mathrm{L}, 0.5 \mathrm{mmol})$ were added to the 
microtube via micropipette. After 3 hours, the biphasic mixture is then passed through a short silica plug and eluted with MTBE. The solvent was then removed by rotavap to give a colorless to pale-yellow oil and the yield of the reaction was determined by ${ }^{1} \mathrm{H}$ NMR ( $96 \%$ conversion, $68 \mathrm{mg}$ (98\% isolated yield)), ee $=90 \%(S)$ by HPLC (Lux Cellulose-1, EtOH:Hexanes $=3: 97,1.0 \mathrm{~mL} / \mathrm{min}$ flow rate, $\lambda=254 \mathrm{~nm}$, retention $(\min ):(R)=19.8,(S)$ $=18.9$ ).

${ }^{1} \mathrm{H}-\mathrm{NMR} \quad\left(400 \mathrm{MHz}, \mathrm{CDCl}_{3}\right) \delta=8.10(\mathrm{brs}, 1 \mathrm{H}), 7.16(\mathrm{td}, J=7.8,1.6 \mathrm{~Hz}, 1 \mathrm{H}), 6.98(\mathrm{dd}, J$ $=7.9,1.2 \mathrm{~Hz}, 1 \mathrm{H}), 6.86-6.82(\mathrm{~m}, 2 \mathrm{H}), 5.03(\mathrm{q}, J=6.6 \mathrm{~Hz}, 1 \mathrm{H}), 3.19$ (brs, $1 \mathrm{H}), 1.56(\mathrm{~d}, J$ $=6.6 \mathrm{~Hz}, 3 \mathrm{H})$

${ }^{13} \mathrm{C}-\mathrm{NMR}\left(100 \mathrm{MHz}, \mathrm{CDCl}_{3}\right) \delta=155.3,129.0,128.7,126.7,120.2,117.1,71.5,23.5$

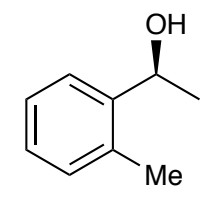

\section{1-(2-methylphenyl)ethanol ${ }^{5}$}

Following ATH Method 1 or 2, $0.8 \mathrm{~mL}$ of a $3.125 \mathrm{M}$ sodium formate solution in water and 2'-methylacetophenone $(66.7 \mu \mathrm{L}, 0.5 \mathrm{mmol})$ were added to the microtube via micropipette. After 3 hours, the biphasic mixture is then passed through a short silica plug and eluted with MTBE. The solvent was then removed by rotavap to give a colorless to pale-yellow oil and the yield of the reaction was determined by ${ }^{1} \mathrm{H}$ NMR ( $27 \%$ conversion), ee $=78 \%$ (S) by HPLC (Lux Cellulose-1, EtOH:Hexanes $=5: 95,1.0$ $\mathrm{mL} / \mathrm{min}$ flow rate, $\lambda=254 \mathrm{~nm}$, retention $(\min ):(\mathrm{R})=7.7,(\mathrm{~S})=7.2)$.

${ }^{1} \mathrm{H}-\mathrm{NMR} \quad\left(400 \mathrm{MHz}, \mathrm{CDCl}_{3}\right) \delta=7.52(\mathrm{dd}, J=7.8,1 \mathrm{H}), 7.24(\mathrm{td}, J=7.8,1.2 \mathrm{~Hz}, 1 \mathrm{H})$, 7.20-7.13 (m, 2H), $5.14(\mathrm{q}, J=6.4 \mathrm{~Hz}, 1 \mathrm{H}), 2.35$ (s, 3H), 1.75 (brs, 1H), 1.47 (d, J = 6.4 $\mathrm{Hz}, 3 \mathrm{H})$

${ }^{13}$ C-NMR $\left(100 \mathrm{MHz}, \mathrm{CDCl}_{3}\right) \delta=144.0,134.4,130.5,127.3,126.5,124.7,67.0,24.1,19.1$

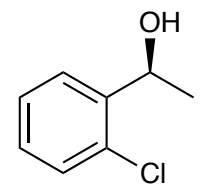

\section{1-(2-chlorophenyl)ethanol ${ }^{9}$}

Following ATH Method 1 or $2,0.8 \mathrm{~mL}$ of a $3.125 \mathrm{M}$ sodium formate solution in water and 2'-chloroacetophenone $(67.7 \mu \mathrm{L}, 0.5 \mathrm{mmol})$ were added to the microtube via micropipette. After 3 hours, the biphasic mixture is then passed through a short silica plug and eluted with MTBE. The solvent was then removed by rotavap to give a pale to light yellow oil and the yield of the reaction was determined by ${ }^{1} \mathrm{H}$ NMR $(99 \%$ conversion, $72 \mathrm{mg}(92 \%$ isolated yield)), ee $=83 \%(S)$ by GC: (Agilent J\&W Cyclodex-B 
$(30 \mathrm{~m} \times 0.25 \mathrm{~mm} \times 0.25 \mu \mathrm{m}), \mathrm{T}=135^{\circ} \mathrm{C}, \mathrm{P}=1.2 \mathrm{~mL} / \mathrm{min}(9.10 \mathrm{psi}) \mathrm{He}$, detector $=\mathrm{FID}$ $230{ }^{\circ} \mathrm{C}$, injection $=230^{\circ} \mathrm{C}$, retention $\left.(\min ):(R)=16.5,(S)=17.6\right)$.

${ }^{1} \mathrm{H}-\mathrm{NMR} \quad\left(400 \mathrm{MHz}, \mathrm{CDCl}_{3}\right) \delta=7.59(\mathrm{dd}, J=7.8,1.3 \mathrm{~Hz}, 1 \mathrm{H}), 7.33-7.28(\mathrm{~m}, 2 \mathrm{H}), 7.21-$ $7.12(\mathrm{~m}, 2 \mathrm{H}), 7.20$ (td, $J=7.6,1.6 \mathrm{~Hz}, 1 \mathrm{H}), 5.29$ (q, $J=6.4 \mathrm{~Hz}, 1 \mathrm{H}), 2.12$ (brs, $1 \mathrm{H}), 1.49$ (d, $J=6.4 \mathrm{~Hz}, 3 \mathrm{H})$

${ }^{13} \mathrm{C}$-NMR $\left(100 \mathrm{MHz}, \mathrm{CDCl}_{3}\right) \delta=143.2,131.9,129.6,128.6,127.4,126.6,67.2,23.7$

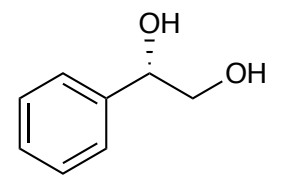

\section{2-hydroxy-1-phenylethanol ${ }^{9}$}

Following ATH Method 1 or 2, $0.8 \mathrm{~mL}$ of a $3.125 \mathrm{M}$ sodium formate solution in water and 2-hydroxyacetophenone $(68.8 \mu \mathrm{L}, 0.5 \mathrm{mmol})$ were added to the microtube via micropipette. After 3 hours, the biphasic mixture is then passed through a short silica plug and eluted with MTBE. The solvent was then removed by rotavap to give a colorless to pale-yellow solid and the yield of the reaction was determined by ${ }^{1} \mathrm{H}$ NMR (92\% conversion), ee $=79 \%(S)$ by HPLC (Lux Cellulose- 1 , EtOH:Hexanes $=5: 95,1.0 \mathrm{~mL} / \mathrm{min}$ flow rate, $\lambda=254 \mathrm{~nm}$, retention $(\mathrm{min}):(\mathrm{R})=16.6,(\mathrm{~S})$ $=15.1$ ).

${ }^{1} \mathrm{H}-\mathrm{NMR} \quad\left(400 \mathrm{MHz}, \mathrm{CDCl}_{3}\right) \delta=7.38-7.29(\mathrm{~m}, 5 \mathrm{H}), 4.84(\mathrm{dt}, J=8.1,3.2 \mathrm{~Hz}, 1 \mathrm{H}), 3.80-$ $3.75(\mathrm{~m}, 1 \mathrm{H}), 3.71-3.65(\mathrm{~m}, 1 \mathrm{H}), 2.50$ (brs, 1H), $2.04(\mathrm{br}, 1 \mathrm{H})$

${ }^{13} \mathrm{C}$-NMR $\left(100 \mathrm{MHz}, \mathrm{CDCl}_{3}\right) \delta=140.7,128.8,128.3,126.3,74.9,68.3$

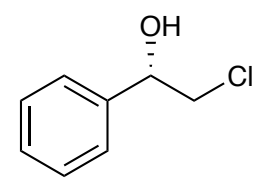

\section{2-chloro-1-phenylethanol ${ }^{9}$}

Following ATH Method 1 or $2,0.8 \mathrm{~mL}$ of a $3.125 \mathrm{M}$ sodium formate solution in water and 2-chloroacetophenone $(78.9 \mathrm{mg}, 0.5 \mathrm{mmol})$ were added to the microtube via micropipette. After 3 hours, the biphasic mixture is then passed through a short silica plug and eluted with MTBE. The solvent was then removed by rotavap to give a pale to light yellow oil and the yield of the reaction was determined by ${ }^{1} \mathrm{H}$ NMR (99\% conversion, $73 \mathrm{mg}(93 \%$ isolated yield)), ee $=92 \%(S)$ by HPLC (Lux Cellulose-1, EtOH:Hexanes $=5: 95,1.0 \mathrm{~mL} / \mathrm{min}$ flow rate, $\lambda=254 \mathrm{~nm}$, retention $(\mathrm{min}):(\mathrm{R})$ $=10.2,(\mathrm{~S})=12.6)$.

${ }^{1} \mathrm{H}-\mathrm{NMR} \quad\left(400 \mathrm{MHz}, \mathrm{CDCl}_{3}\right) \delta=7.41-7.31(\mathrm{~m}, 5 \mathrm{H}), 4.91(\mathrm{dd}, \mathrm{J}=8.8,3.4 \mathrm{~Hz}, 1 \mathrm{H}), 3.75$ (dd, $J=11.2,3.4 \mathrm{~Hz}, 1 \mathrm{H}$ ), 3.65 (dd, $J=11.2,8.8 \mathrm{~Hz}, 1 \mathrm{H}), 2.63$ (brs, 1H) 
${ }^{13}$ C-NMR $\left(100 \mathrm{MHz}, \mathrm{CDCl}_{3}\right) \delta=140.1,128.9,128.7,126.3,74.3,51.2$

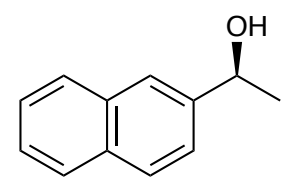

\section{1-(2-naphthyl)ethanol ${ }^{6}$}

Following ATH Method 1 or $2,0.8 \mathrm{~mL}$ of a $3.125 \mathrm{M}$ sodium formate solution in water and 2-acetonapthone $(86.0 \mathrm{mg}, 0.5 \mathrm{mmol})$ were added to the microtube via micropipette. After 3 hours, the biphasic mixture is then passed through a short silica plug and eluted with MTBE. The solvent was then removed by rotavap to give a colorless to pale-yellow solid and the yield of the reaction was determined by ${ }^{1} \mathrm{H}$ NMR ( $85 \%$ conversion), ee $=91 \%$ (S) by GC: (Agilent J\&W Cyclodex$\mathrm{B}(30 \mathrm{~m} \times 0.25 \mathrm{~mm} \times 0.25 \mu \mathrm{m}), \mathrm{T}=160^{\circ} \mathrm{C}, \mathrm{P}=2.5 \mathrm{~mL} / \mathrm{min}(16.5 \mathrm{psi}) \mathrm{He}$, detector $=\mathrm{FID}$ $230{ }^{\circ} \mathrm{C}$, injection $=230{ }^{\circ} \mathrm{C}$, retention $\left.(\min ):(R)=32.6,(S)=33.1\right)$.

${ }^{1} \mathrm{H}-\mathrm{NMR} \quad\left(400 \mathrm{MHz}, \mathrm{CDCl}_{3}\right) \delta=7.86-7.82(\mathrm{~m}, 4 \mathrm{H}), 7.53-7.44(\mathrm{~m}, 3 \mathrm{H}), 5.08(\mathrm{q}, J=6.4$ $\mathrm{Hz}, 1 \mathrm{H}), 1.89(\mathrm{~s}, 1 \mathrm{H}), 1.59(\mathrm{~d}, J=6.4 \mathrm{~Hz}, 1 \mathrm{H})$

${ }^{13}$ C-NMR $\left(100 \mathrm{MHz}, \mathrm{CDCl}_{3}\right) \delta=143.4,133.6,133.1,128.5,128.2,127.9,126.4,126.0$, $124.0,70.8,25.4$

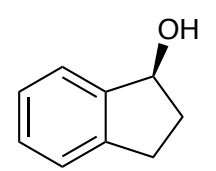

\section{1-indanol ${ }^{6}$}

Following ATH Method 1 or $2,0.8 \mathrm{~mL}$ of a $3.125 \mathrm{M}$ sodium formate solution in water and 1-indanone $(66.1 \mathrm{mg}, 0.5 \mathrm{mmol})$ were added to the microtube via micropipette. After 3 hours, the biphasic mixture is then passed through a short silica plug and eluted with MTBE. The solvent was then removed by rotavap to give a colorless to pale-yellow solid and the yield of the reaction was determined by ${ }^{1} \mathrm{H}$ NMR $(69 \%$ conversion), ee $=95 \%(S)$ by HPLC (Lux Cellulose-1, EtOH:Hexanes $=5: 95,1.0 \mathrm{~mL} / \mathrm{min}$ flow rate, $\lambda=254 \mathrm{~nm}$, retention $(\mathrm{min}):(\mathrm{R})=8.9,(\mathrm{~S})=8.1)$.

${ }^{1} \mathrm{H}-\mathrm{NMR} \quad\left(300 \mathrm{MHz}, \mathrm{CDCl}_{3}\right) \delta=7.44-7.40(\mathrm{~m}, 1 \mathrm{H}), 7.28-7.21(\mathrm{~m}, 3 \mathrm{H}), 5.24(\mathrm{q}, J=6.8$ $\mathrm{Hz}, 1 \mathrm{H}$ ), 3.05 (ddd, $J=15.9,8.6,4.8 \mathrm{~Hz}, 1 \mathrm{H}), 2.88-2.77(\mathrm{~m}, 1 \mathrm{H}), 2.55-2.44(\mathrm{~m}, 1 \mathrm{H}), 2.00-$ $1.89(\mathrm{~m}, 1 \mathrm{H}), 1.84(\mathrm{~d}, J=7.1 \mathrm{~Hz}, 1 \mathrm{H})$

${ }^{13} \mathrm{C}$-NMR $\left(100 \mathrm{MHz}, \mathrm{CDCl}_{3}\right) \delta=145.2,143.5,128.5,126.9,125.1,124.4,76.6,36.1,30.0$ 


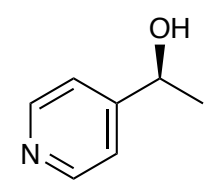

\section{1-(4-pyridyl)ethanol ${ }^{8}$}

Following ATH Method $1,0.8 \mathrm{~mL}$ of a $3.125 \mathrm{M}$ sodium formate solution in water and 4-acetylpyridine $(57.0 \mu \mathrm{L}, 0.5 \mathrm{mmol})$ were added to the microtube via micropipette. The biphasic mixture is then transferred to a separatory funnel and the organics are extracted twice with either MTBE or DCM. The solvent was then removed by rotavap to give an orange oil and the yield of the reaction was determined by ${ }^{1} \mathrm{H}$ NMR (37\% conversion), ee $=91 \%(S)$ by GC: (Agilent J\&W Cyclodex-B $(30 \mathrm{~m} \times 0.25 \mathrm{~mm} \times$ $0.25 \mu \mathrm{m}), \mathrm{T}=135^{\circ} \mathrm{C}, \mathrm{P}=1.1 \mathrm{~mL} / \mathrm{min}(8.07 \mathrm{psi}) \mathrm{He}$, detector $=\mathrm{FID} 230^{\circ} \mathrm{C}$, injection $=$ $230^{\circ} \mathrm{C}$, retention $\left.(\mathrm{min}):(R)=32.4,(S)=33.2\right)$.

${ }^{1} \mathrm{H}-\mathrm{NMR} \quad\left(300 \mathrm{MHz}, \mathrm{CDCl}_{3}\right) \delta=8.43(\mathrm{dd}, J=4.5,1.6 \mathrm{~Hz}, 2 \mathrm{H}), 7.28$ (ddd, $J=4.5,1.6$, $0.5 \mathrm{~Hz}, 2 \mathrm{H}$ ), $4.87(\mathrm{q}, J=6.5 \mathrm{~Hz}, 1 \mathrm{H}), 3.86(\mathrm{br}, 1 \mathrm{H}), 1.47(\mathrm{~d}, J=6.6 \mathrm{~Hz}, 2 \mathrm{H})$

${ }^{13} \mathrm{C}$-NMR $\left(100 \mathrm{MHz}, \mathrm{CDCl}_{3}\right) \delta=155.6,149.6,120.7,68.8,25.3$<smiles>C[C@@H](O)c1cccnc1</smiles>

\section{1-(3-pyridyl)ethanol ${ }^{8}$}

Following ATH Method 1 or 2, $0.8 \mathrm{~mL}$ of a $3.125 \mathrm{M}$ sodium formate solution in water and 3-acetylpyridine $(56.7 \mu \mathrm{L}, 0.5 \mathrm{mmol})$ were added to the microtube via micropipette. The biphasic mixture is then transferred to a separatory funnel and the organics are extracted twice with either MTBE or DCM. The solvent was then removed by rotavap to give an orange-colored oil, and the yield of the reaction was determined by ${ }^{1} \mathrm{H}$ NMR (37\% conversion), ee $=90 \%$ (S) by GC: (Agilent J\&W Cyclodex$\mathrm{B}(30 \mathrm{~m} \times 0.25 \mathrm{~mm} \times 0.25 \mu \mathrm{m}), \mathrm{T}=135^{\circ} \mathrm{C}, \mathrm{P}=1.1 \mathrm{~mL} / \mathrm{min}(8.07 \mathrm{psi}) \mathrm{He}$, detector $=\mathrm{FID}$ $230{ }^{\circ} \mathrm{C}$, injection $=230{ }^{\circ} \mathrm{C}$, retention $(R)=29.0,(S)=29.9$ ).

${ }^{1} \mathrm{H}-\mathrm{NMR} \quad\left(400 \mathrm{MHz}, \mathrm{CDCl}_{3}\right) \delta=8.44(\mathrm{~d}, J=2.1 \mathrm{~Hz}, 1 \mathrm{H}), 8.36(\mathrm{dd}, J=4.8,1.6 \mathrm{~Hz}, 1 \mathrm{H})$, $7.71(\mathrm{dt}, J=7.9,1.7 \mathrm{~Hz}, 1 \mathrm{H}), 7.23(\mathrm{dd}, J=7.8,4.8 \mathrm{~Hz}, 1 \mathrm{H}), 4.89(\mathrm{dq}, J=6.4,4.1 \mathrm{~Hz}, 1 \mathrm{H})$, $4.12(\mathrm{~d}, J=3.7 \mathrm{~Hz}, 1 \mathrm{H}), 1.48(\mathrm{~d}, J=6.5 \mathrm{~Hz}, 2 \mathrm{H})$

${ }^{13} \mathrm{C}$-NMR $\left(100 \mathrm{MHz}, \mathrm{CDCl}_{3}\right) \delta=148.4,147.3,141.7,133.6,123.7,67.8,25.4$

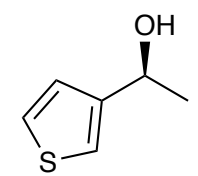

\section{1-(thiophen-3-yl)ethanol ${ }^{8}$}

Following ATH Method 1 or $2,0.8 \mathrm{~mL}$ of a $3.125 \mathrm{M}$ sodium formate solution in water and 3-acetylthiophene $(64.4 \mathrm{mg}, 0.5 \mathrm{mmol})$ were added to the microtube via micropipette. After 3 hours, the biphasic mixture is then passed through a 
short silica plug and eluted with MTBE. The solvent was then removed by rotavap to give a colorless to give a pale to light yellow oil and the yield of the reaction was determined by ${ }^{1} \mathrm{H}$ NMR (77\% conversion), ee $=92 \%(S)$ by GC: (Agilent J\&W Cyclodex-B (30 $\mathrm{m} \mathrm{x}$ $0.25 \mathrm{~mm} \times 0.25 \mu \mathrm{m}), \mathrm{T}=115^{\circ} \mathrm{C}, \mathrm{P}=1.0 \mathrm{~mL} / \mathrm{min}(6.95 \mathrm{psi}) \mathrm{He}$, detector $=\mathrm{FID} 230^{\circ} \mathrm{C}$, injection $=230{ }^{\circ} \mathrm{C}$, retention $\left.(\min ):(R)=35.7,(S)=37.0\right)$.

${ }^{1} \mathrm{H}-\mathrm{NMR} \quad\left(400 \mathrm{MHz}, \mathrm{CDCl}_{3}\right) \delta=7.30(\mathrm{dd}, J=5.0,3.0 \mathrm{~Hz}, 1 \mathrm{H}), 7.19(\mathrm{~d}, J=2.6 \mathrm{~Hz}, 1 \mathrm{H})$, 7.10 (dd, $J=5.0,1.1 \mathrm{~Hz}, 1 \mathrm{H}$ ), $4.98(\mathrm{~d}, J=6.4 \mathrm{~Hz}, 1 \mathrm{H}$ ), 1.83 (brs, $1 \mathrm{H}), 1.53$ (d, $J=6.5 \mathrm{~Hz}$, $3 \mathrm{H})$

${ }^{13} \mathrm{C}$-NMR $\left(100 \mathrm{MHz}, \mathrm{CDCl}_{3}\right) \delta=147.5,126.4,125.8,120.4,66.8,24.7$

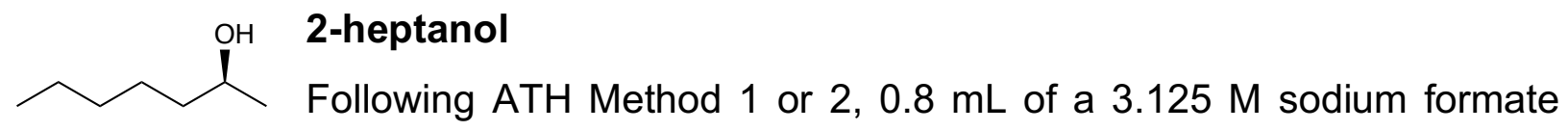
solution in water and 2-heptanone $(72.1 \mu \mathrm{L}, 0.5 \mathrm{mmol})$ were added to the microtube via micropipette. After 24 hours, the biphasic mixture is then transferred to a separatory funnel and diluted with $\mathrm{Et}_{2} \mathrm{O}(25 \mathrm{~mL})$. The organics were washed with saturated brine (2 x $10 \mathrm{~mL}$ ), dried over $\mathrm{MgSO}_{4}$, filtered, and the ether was removed using a warm water bath to give a yellow liquid and the yield of the reaction was determined by ${ }^{1} \mathrm{H}$ NMR $(77 \%$ conversion). The crude alcohol is then converted to the corresponding acetate using acetic anhydride $(200 \mu \mathrm{L})$ and a catalytic amount of DMAP (5-10 mg) and left to stir overnight at room temperature. The reaction mixture was transferred to a separatory funnel, washed with water $(10 \mathrm{~mL})$ and the organics were extracted with DCM $(2 \times 20$ $\mathrm{mL}$ ). The combined organics were washed with saturated brine (10 $\mathrm{mL})$, dried over $\mathrm{MgSO}_{4}$, filtered, and the DCM was removed using a warm water bath to give a yellow oil, ee $=17 \%(S)$ by GC: (Agilent J\&W Cyclodex-B $(30 \mathrm{~m} \times 0.25 \mathrm{~mm} \times 0.25 \mu \mathrm{m}), \mathrm{T}=80^{\circ} \mathrm{C}$, $\mathrm{P}=1.1 \mathrm{~mL} / \mathrm{min}(6.59 \mathrm{psi}) \mathrm{He}$, detector $=\mathrm{FID} 230^{\circ} \mathrm{C}$, injection $=230^{\circ} \mathrm{C}$, retention $(\mathrm{min})$ : $(R)=35.4,(S)=40.8)$.

${ }^{1} \mathrm{H}-\mathrm{NMR} \quad\left(400 \mathrm{MHz} \mathrm{CDCl}_{3}\right) \delta=3.83-3.75(\mathrm{~m}, 1 \mathrm{H}), 1.50-1.26(\mathrm{~m}, 9 \mathrm{H}), 1.18(\mathrm{~d}, \mathrm{~J}=6.2$, $3 \mathrm{H}), 0.89(\mathrm{t}, J=6.9 \mathrm{~Hz}, 1 \mathrm{H}), 7.10(\mathrm{dd}, J=5.0,1.1 \mathrm{~Hz}, 1 \mathrm{H}), 4.98(\mathrm{~d}, J=6.4 \mathrm{~Hz}, 1 \mathrm{H}), 1.83$ (brs, $1 \mathrm{H}), 1.53(\mathrm{~d}, J=6.5 \mathrm{~Hz}, 3 \mathrm{H})$

${ }^{13} \mathrm{C}-\mathrm{NMR}\left(100 \mathrm{MHz}, \mathrm{CDCl}_{3}\right) \delta=68.4,39.6,32.1,25.7,23.7,22.8,14.2$ 


\section{Supplemental Data and Figures}

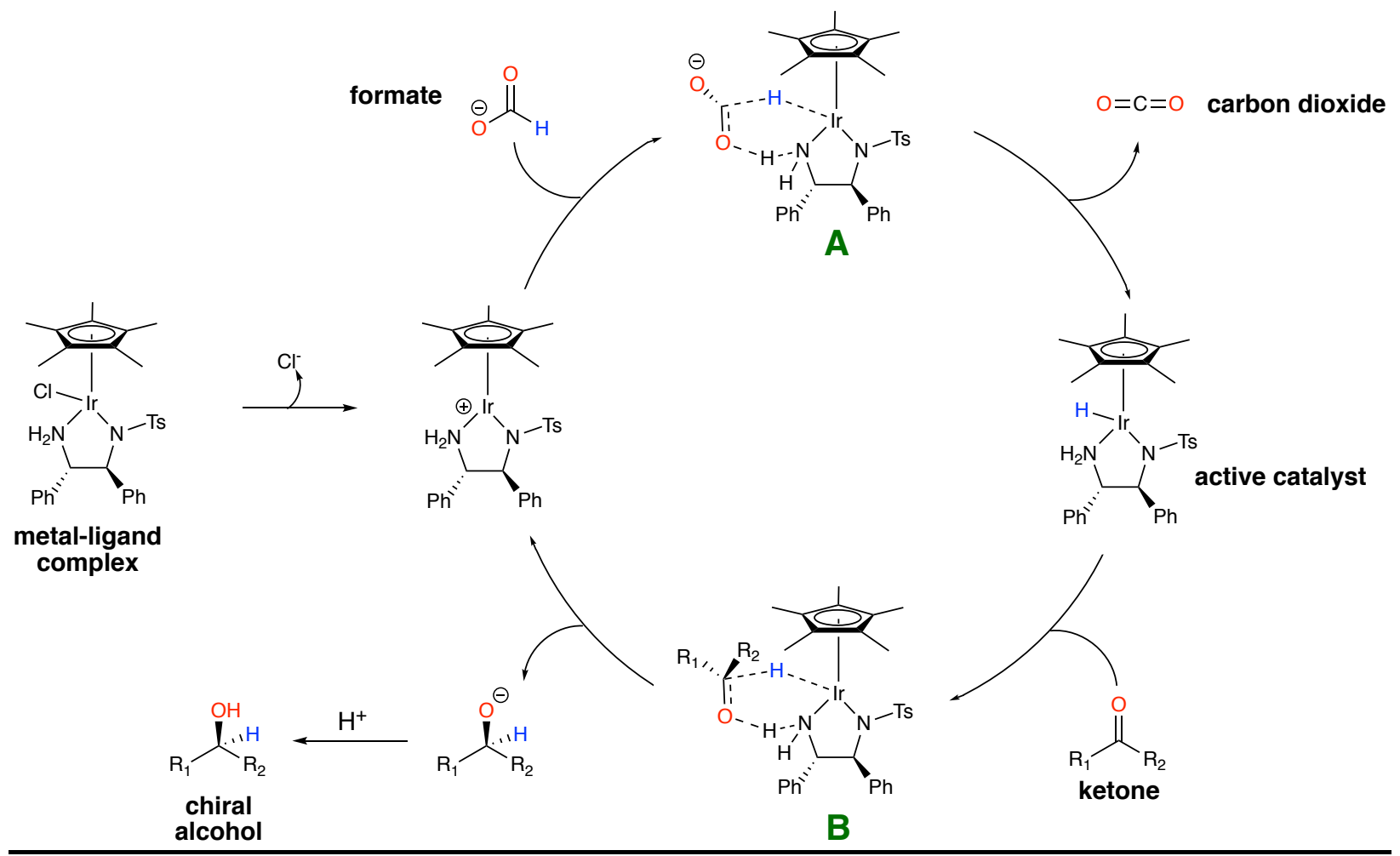

Scheme S1. General ATH mechanism with use of sodium formate as the reducing agent. 

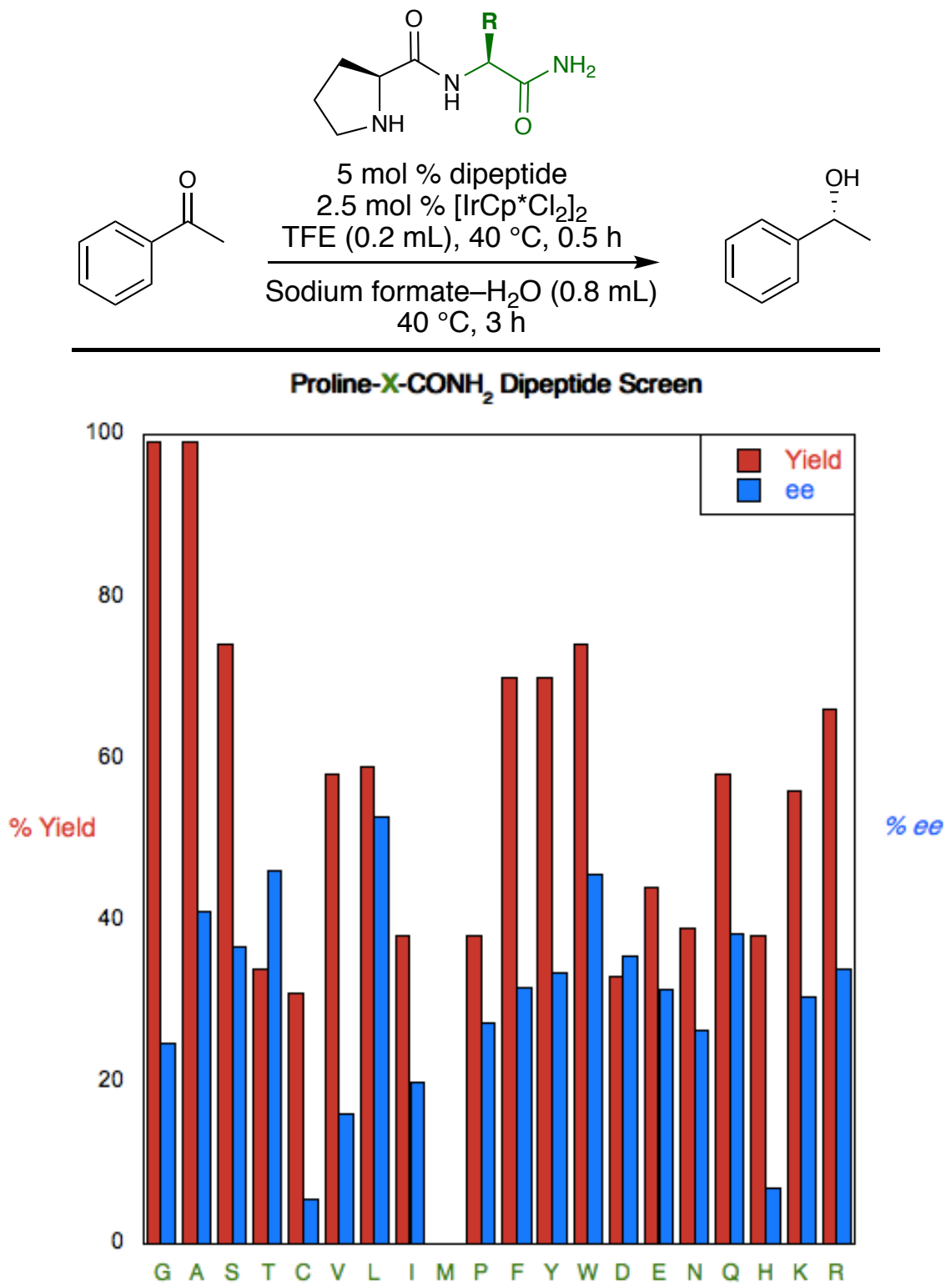

Figure S1. Performance of peptide catalysts in transfer hydrogenation. Yield (\%, red) and enantiomeric excess (\%, blue) of dipeptides of the general formula $\mathrm{HN}-\mathrm{Pro}-\mathrm{X}-\mathrm{CONH} \mathrm{CH}_{2}$ (where $\mathrm{X}$ includes all 20 genetically encoded amino acids) in transfer hydrogenation of acetophenone ( $5 \mathrm{~mol} \%$ of catalyst, $3.125 \mathrm{M}$ sodium formate). 


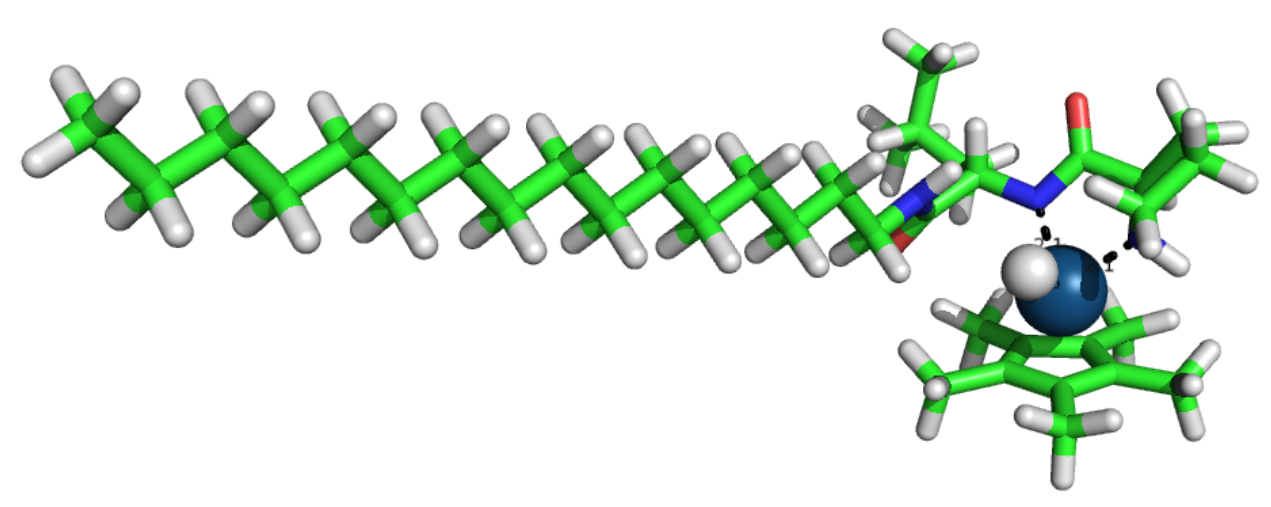

Figure S2. DFT model of $C p^{*} \operatorname{Ir}(\mathrm{H})\left({ }^{\mathrm{D} P L}-\mathrm{C}_{16}\right)$. The Ir-N bond lengths are $2.122 \AA, 2.114 \AA$ for Ir-N(Pro) and Ir-N(Leu), and Ir-H bond length is $1.600 \AA$.
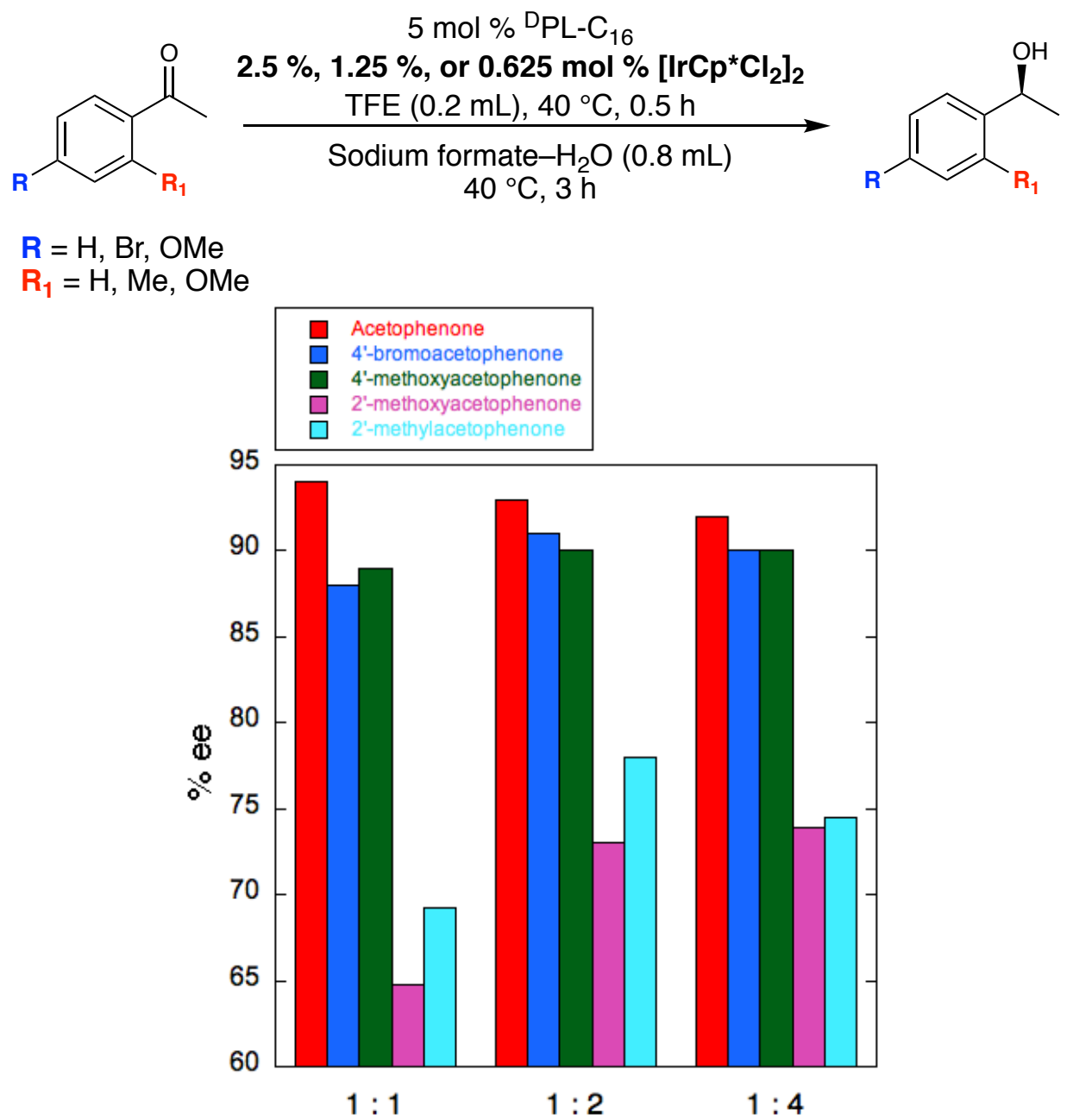

Figure S3. Enantiomeric selectivity in transfer hydrogenation promoted by ${ }^{\mathrm{D} L-\mathrm{C}_{16}}$ at various metal:peptide ratios (ligand concentration was kept constant at $0.025 \mathrm{mM}$ ). A $3.125 \mathrm{M}$ solution of sodium formate was used. 


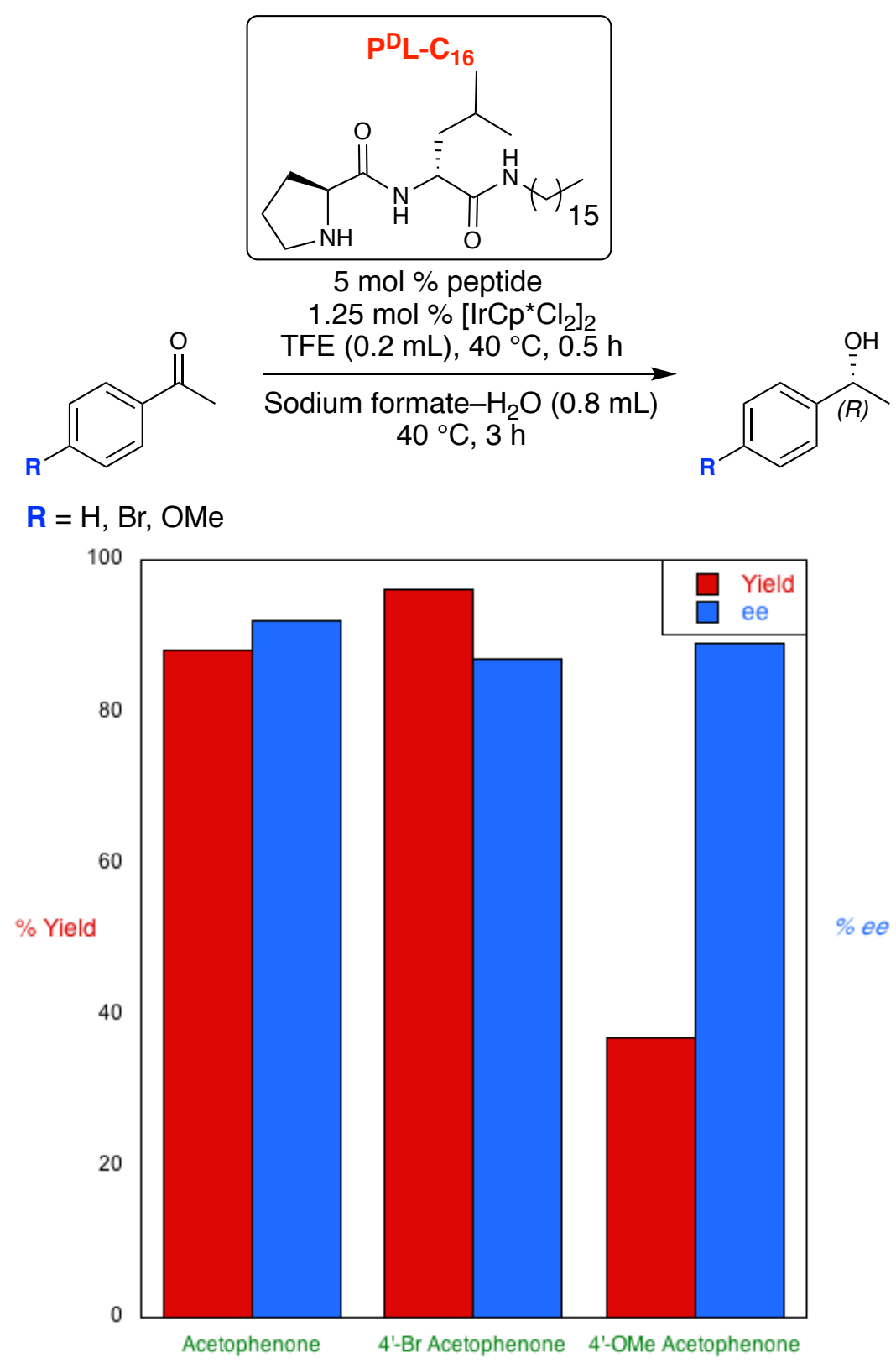

Figure S4. Enantiomeric selectivity in transfer hydrogenation promoted by $\mathrm{P}^{\mathrm{D}} \mathrm{L}-\mathrm{C}_{16}$ using the conditions outlined in the scheme. Reported yields were obtained by ${ }^{1} \mathrm{H}-\mathrm{NMR}$ and ee obtained by chiral HPLC. 

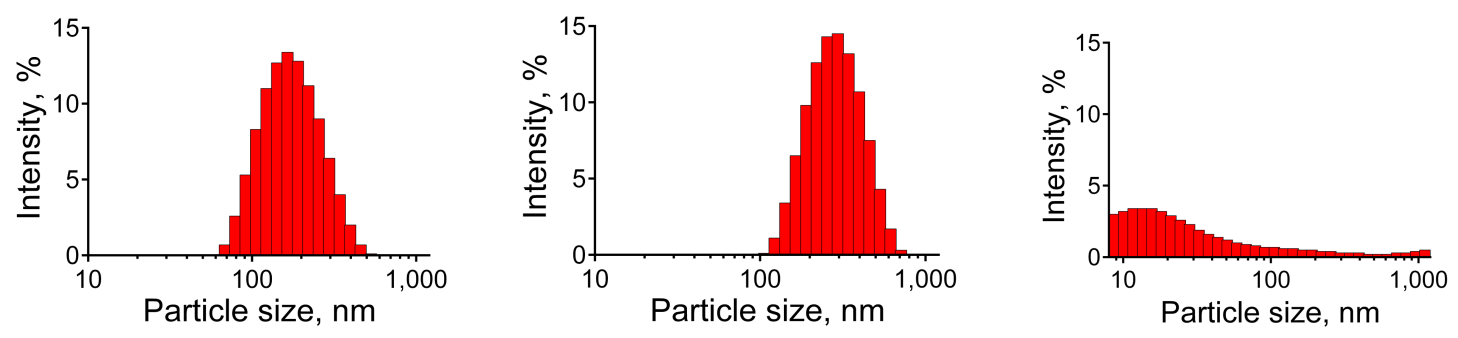

Figure S5. Dynamic light scattering data for ${ }^{D P L}-C_{16}\left(0.02 \mathrm{mM}\right.$ solution in $\left.20 \% \mathrm{TFE} / \mathrm{H}_{2} \mathrm{O}\right)$ (left), $\mathrm{Cp}^{*} \operatorname{Ir}(\mathrm{H})\left({ }^{\mathrm{DPL}}-\mathrm{C}_{16}\right)\left(0.02 \mathrm{mM}\right.$ solution in $\left.20 \% \mathrm{TFE} / \mathrm{H}_{2} \mathrm{O}\right)\left(\right.$ middle) and $\mathrm{Cp}{ }^{*} \operatorname{Ir}(\mathrm{H})\left({ }^{\mathrm{DPL}}-\right.$ $\left.\mathrm{C}_{16}\right)\left(0.02 \mathrm{mM}\right.$ solution in $20 \%$ toluene $\left./ \mathrm{H}_{2} \mathrm{O}\right)($ right $)$.

a

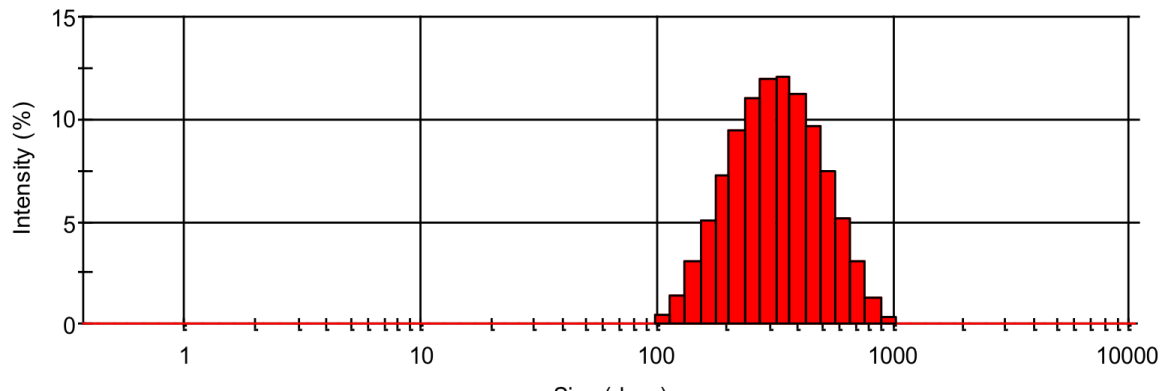

b

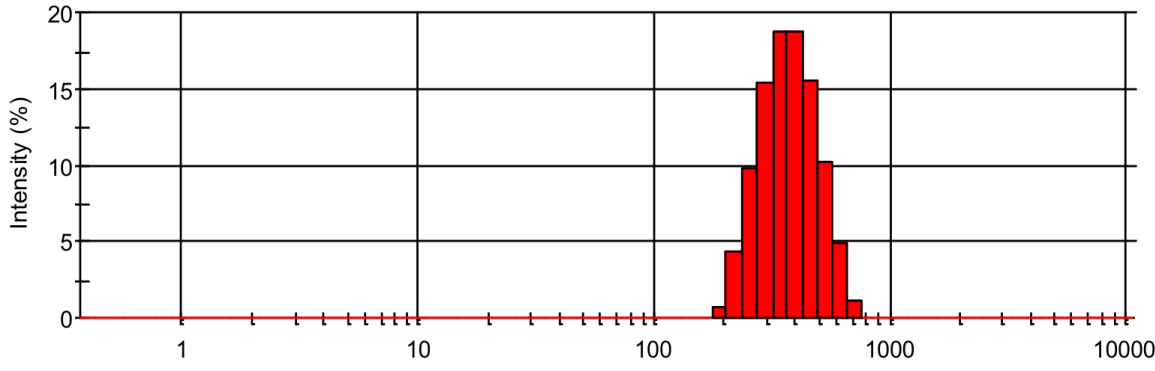

C

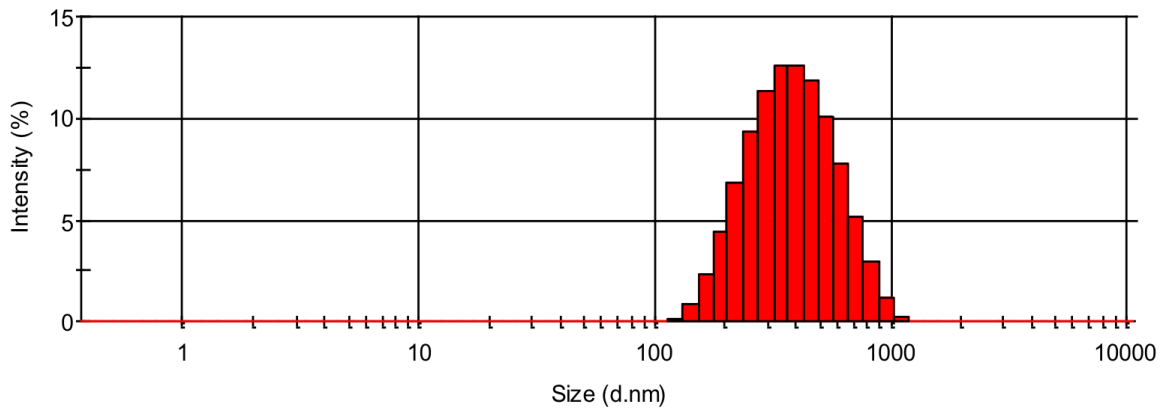

Figure S6. Dynamic light scattering data for $C p^{*} \operatorname{Ir}(\mathrm{H})\left({ }^{\mathrm{D} P L}-\mathrm{C}_{16}\right)$ at varying concentrations in $20 \% \mathrm{EtOH} / \mathrm{H}_{2} \mathrm{O}$ a) $(50 \mu \mathrm{M}$ solution) b) $(75 \mu \mathrm{M}$ solution $)$ c) $(100 \mu \mathrm{M}$ solution $)$. 


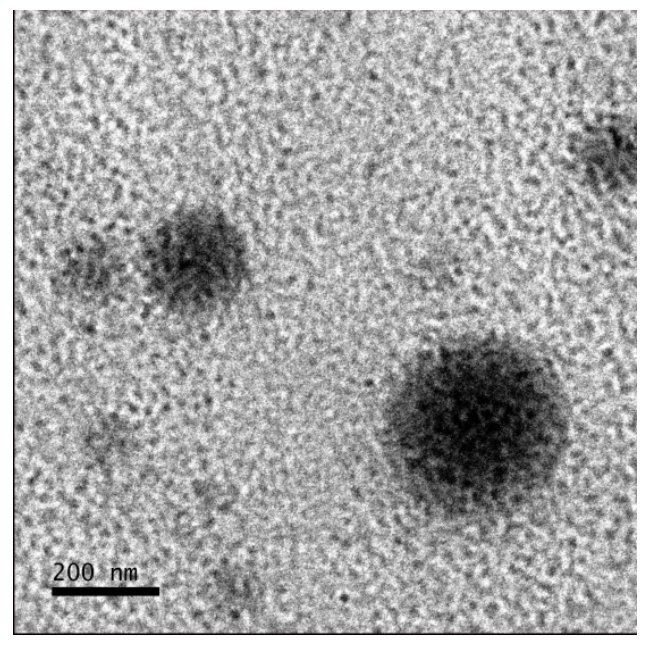

Figure S7. TEM image showing self-assembled micelle structures formed by $C p^{*} \operatorname{Ir}(\mathrm{H})\left({ }^{\mathrm{DPL}}-\mathrm{C}_{16}\right)$ prepared in $20 \% \mathrm{TFE} / \mathrm{H}_{2} \mathrm{O}$ with $20 \mathrm{mM}$ phosphate buffer. Sample preparation: a stock solution of ${ }^{D P L}-\mathrm{C}_{16}$ with $\left[\mathrm{IrCp}^{*} \mathrm{Cl}_{2}\right]_{2}$ was made at $25 \mathrm{mM}$ peptide and $12.5 \mathrm{mM}$ metal in TFE. A diluted $400 \mu \mathrm{M}$ solution was prepared by transferring $16 \mu \mathrm{L}$ of stock solution into $184 \mu \mathrm{L}$ TFE, followed by addition of $800 \mu \mathrm{L}$ with a $100 \mathrm{mM}$ phosphate buffer $(\mathrm{pH}=7.0)$ to give a final concentration of $20 \% \mathrm{TFE} / \mathrm{H}_{2} \mathrm{O}$ with $20 \mathrm{mM}$ phosphate buffer.<smiles>CC(=O)c1ccccc1</smiles>

1)

$5 \mathrm{~mol} \%$ peptide $\underset{\text { Sodium formate }-\mathrm{H}_{2} \mathrm{O}(0.8 \mathrm{~mL})}{\stackrel{2.5 \mathrm{~mol} \%\left[\mathrm{IrCp} \mathrm{Cl}_{2}\right]_{2}}{\text { Toluene }(0.2 \mathrm{~mL}), 100^{\circ} \mathrm{C}, 0.5 \mathrm{~h}}}$ acetophenone $(0.5 \mathrm{mmol})$ $40^{\circ} \mathrm{C}, 3 \mathrm{~h}$

Table S1. Transfer hydrogenation results of peptide catalysts in toluene-water mixtures $(20: 80)$ at conditions specified in the scheme above, peptide concentration was 0.025 mM. Reported yields were obtained by ${ }^{1} \mathrm{H}-\mathrm{NMR}$ and ee by chiral HPLC.

\begin{tabular}{c|cc} 
LIGAND & Yield (\%) & ee (\%) \\
${ }^{D} P L-C_{16}$ & 19 & $69(S)$ \\
${ }^{D} P L-N_{2}$ & $<1$ & n.d.
\end{tabular}




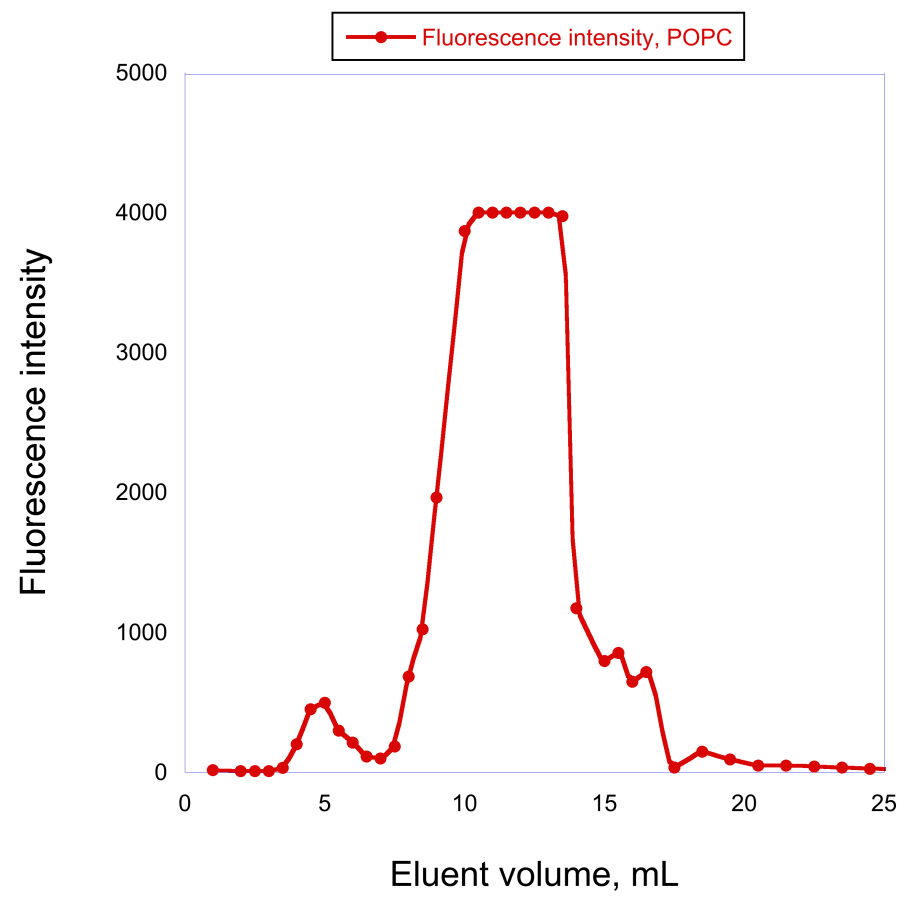

Figure S8. Elution profile of mixtures containing POPC small unilamellar vesicles (red) as described in detail in the methods section above. The eluent was $50 \mathrm{mM}$ HEPES pH 7.0. Fluorescence intensity of entrapped Tb-DPA complex was measured at $490 \mathrm{~nm}$ (excitation wavelength at $270 \mathrm{~nm}$ ). Vesicles elute at $\sim 5 \mathrm{~mL}$ under these conditions. 


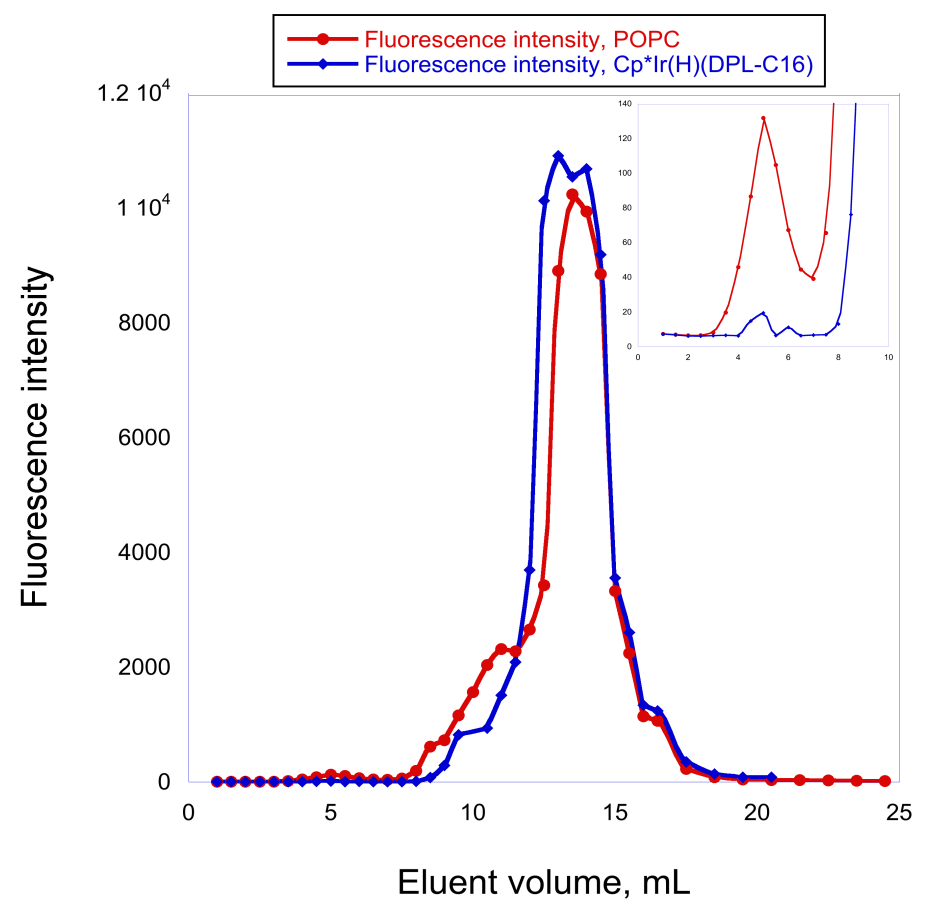

Figure S9. Elution profile of mixtures containing POPC small unilamellar vesicles (red) and supramolecular assemblies formed by $\mathrm{Cp}{ }^{*} \operatorname{Ir}(\mathrm{H})\left({ }^{\left.\mathrm{PPL}-\mathrm{C}_{16}\right)}\right.$ (blue) as described in detail in the methods section above. The eluent was $3.125 \mathrm{M}$ sodium formate. Fluorescence intensity of entrapped Tb-DPA complex was measured at $490 \mathrm{~nm}$ (excitation wavelength at $270 \mathrm{~nm}$ ). Vesicles elute at $\sim 5 \mathrm{~mL}$ under these conditions. This benchmark was confirmed by performing the same experiment using POPC vesicles at much lower (50 $\mathrm{mM}$ ) concentration of buffer (Figure S8 above) - vesicles stability is much higher under these conditions. 


\section{GC and HPLC Chromatograms}

Note 1: Absolute configurations in all cases were determined based on results obtained from ATH reactions using $C p^{*} \operatorname{Ir}(T s D P E N)$.

Note 2: During the course of this work, the GC chiral capillary column was replaced and some chromatograms may show noticeable shifts in retention time.

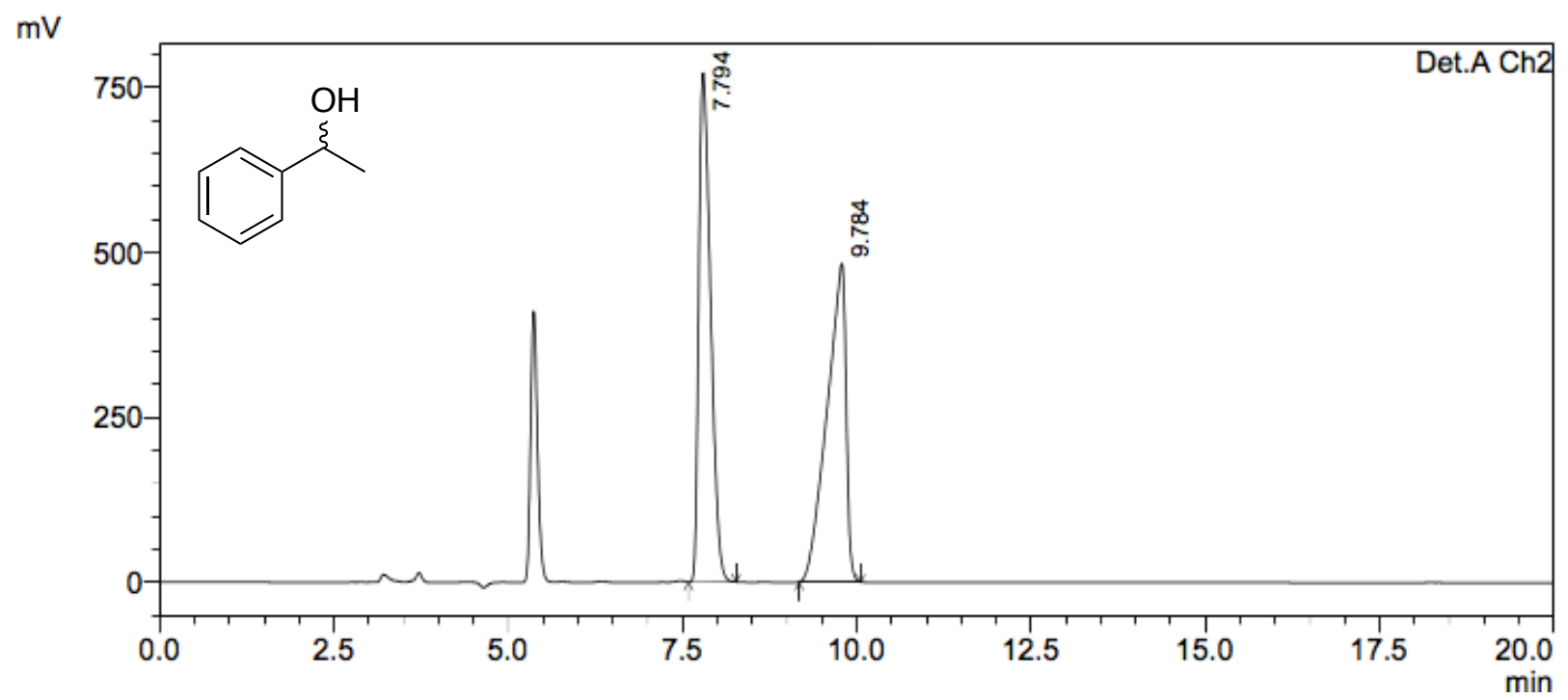

Detector A Ch2 254nm
\begin{tabular}{|r|r|r|r|r|r|}
\hline Peak\# & Ret. Time & \multicolumn{1}{|c|}{ Area } & Height & \multicolumn{1}{|c|}{ Area $\%$} & Height \% \\
\hline 1 & 7.794 & 9067680 & 771094 & 49.760 & 61.516 \\
\hline 2 & 9.784 & 9155250 & 482385 & 50.240 & 38.484 \\
\hline Total & & 18222930 & 1253478 & 100.000 & 100.000 \\
\hline
\end{tabular}

Figure S10. HPLC chromatogram of the reaction mixture produced by $\mathrm{NaBH}_{4}$ reduction of acetophenone. 


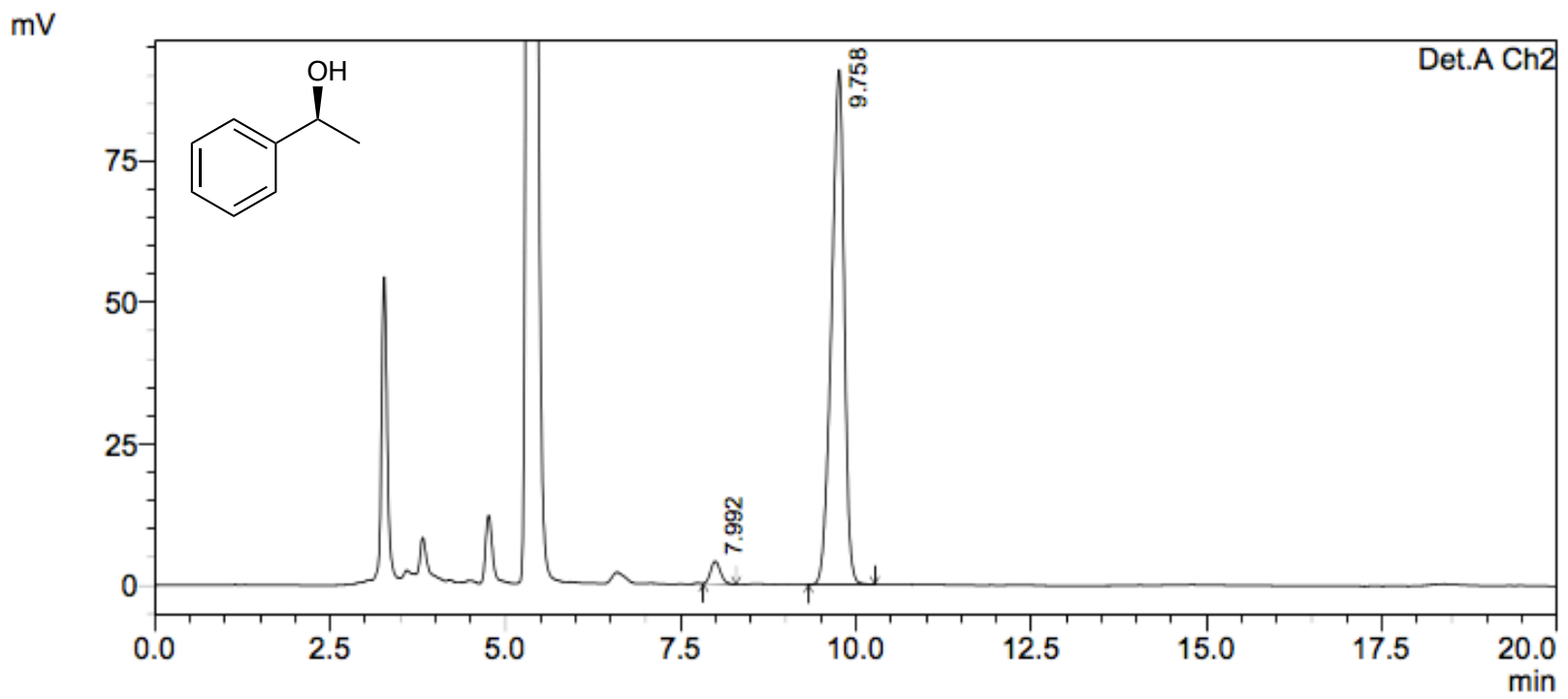

Detector A Ch2 254nm

\begin{tabular}{|r|r|r|r|r|r|}
\hline \multicolumn{1}{|c|}{ Peak\# } & \multicolumn{1}{c|}{ Ret. Time } & \multicolumn{1}{c|}{ Area } & \multicolumn{1}{c|}{ Height } & \multicolumn{1}{c|}{ Area \% } & \multicolumn{1}{c|}{ Height \% } \\
\hline 1 & 7.992 & 38923 & 3974 & 3.282 & 4.191 \\
\hline 2 & 9.758 & 1147083 & 90859 & 96.718 & 95.809 \\
\hline Total & & 1186006 & 94833 & 100.000 & 100.000 \\
\hline
\end{tabular}

Figure S11. HPLC chromatogram of the reaction mixture produced by ATH of acetophenone catalyzed by $\mathrm{Cp}{ }^{*} \operatorname{Ir}\left({ }^{\mathrm{DPL}}-\mathrm{C}_{16}\right)$.

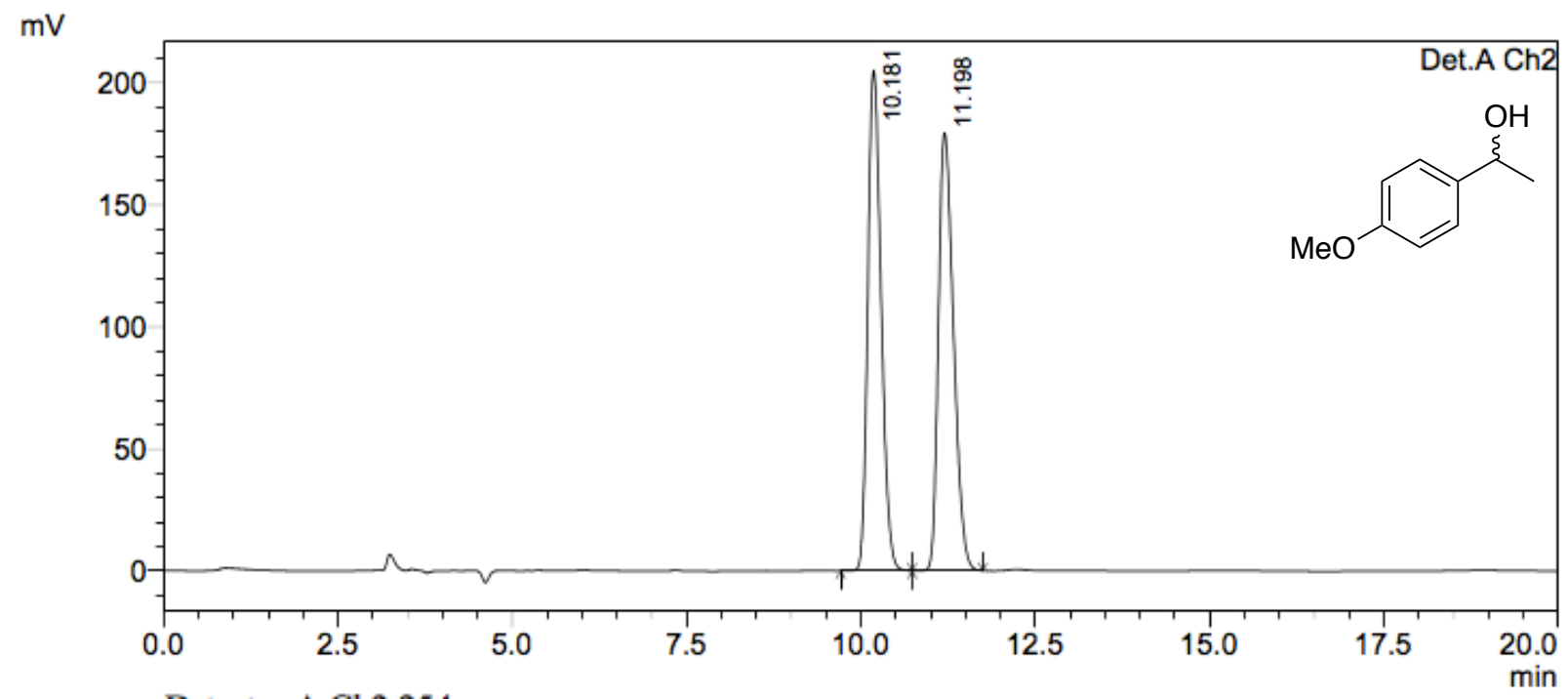

Detector A Ch2 254nm

\begin{tabular}{|r|r|r|r|r|r|}
\hline \multicolumn{1}{|c|}{ Peak\# } & \multicolumn{1}{|c|}{ Ret. Time } & \multicolumn{1}{c|}{ Area } & \multicolumn{1}{c|}{ Height } & \multicolumn{1}{c|}{ Area \% } & \multicolumn{1}{c|}{ Height \% } \\
\hline 1 & 10.181 & 2720274 & 205008 & 50.141 & 53.341 \\
\hline 2 & 11.198 & 2704986 & 179326 & 49.859 & 46.659 \\
\hline Total & & 5425260 & 384334 & 100.000 & 100.000 \\
\hline
\end{tabular}

Figure S12. HPLC chromatogram of the reaction mixture produced by $\mathrm{NaBH}_{4}$ reduction of 4'-methoxyacetophenone. 


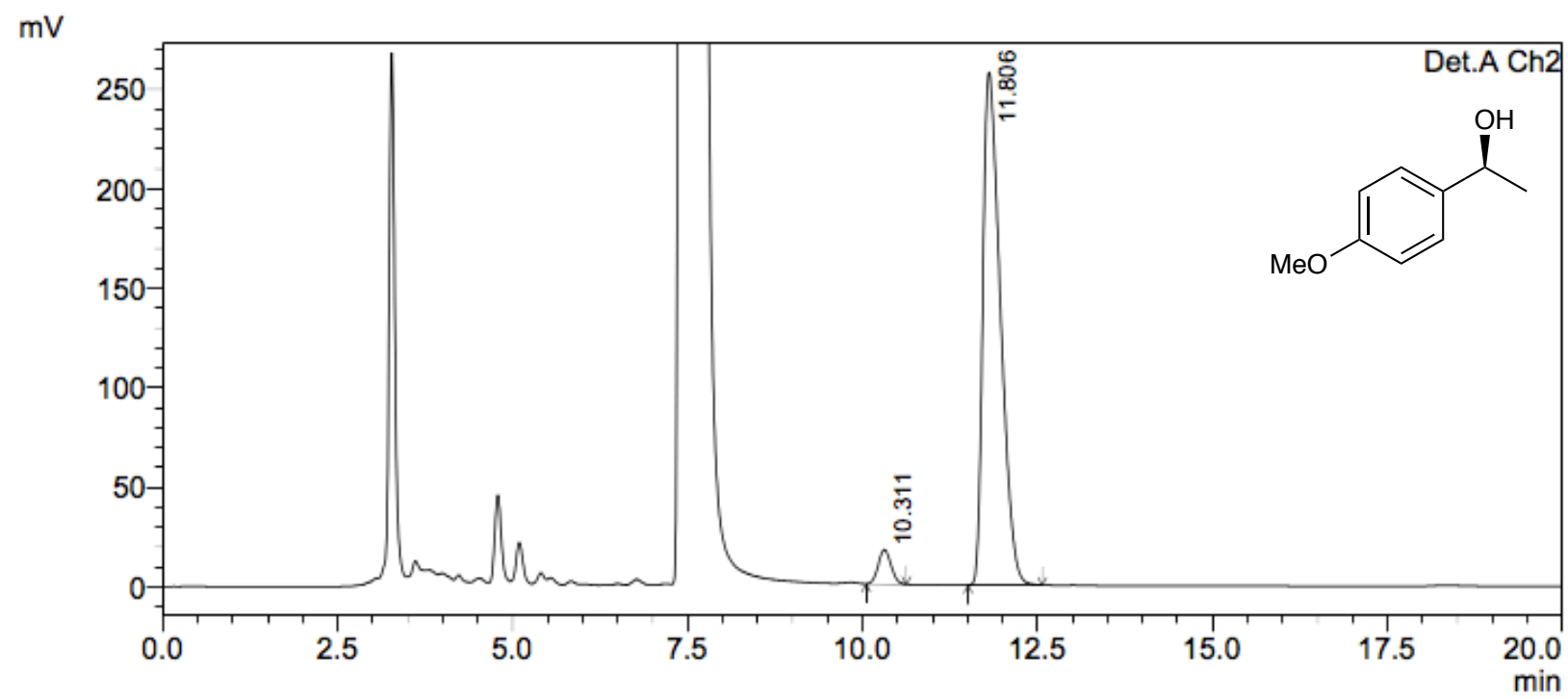

Detector A Ch2 254nm

\begin{tabular}{|r|r|r|r|r|r|}
\hline \multicolumn{1}{|c|}{ Peak\# } & Ret. Time & \multicolumn{1}{c|}{ Area } & Height & \multicolumn{1}{c|}{ Area \% } & \multicolumn{1}{c|}{ Height \% } \\
\hline 1 & 10.311 & 218743 & 17224 & 4.649 & 6.267 \\
\hline 2 & 11.806 & 4486255 & 257589 & 95.351 & 93.733 \\
\hline Total & & 4704999 & 274813 & 100.000 & 100.000 \\
\hline
\end{tabular}

Figure S13. HPLC chromatogram of the reaction mixture produced by ATH of 4'methoxyacetophenone catalyzed by $\mathrm{Cp}{ }^{*} \operatorname{Ir}\left({ }^{\mathrm{D} P L}-\mathrm{C}_{16}\right)$.

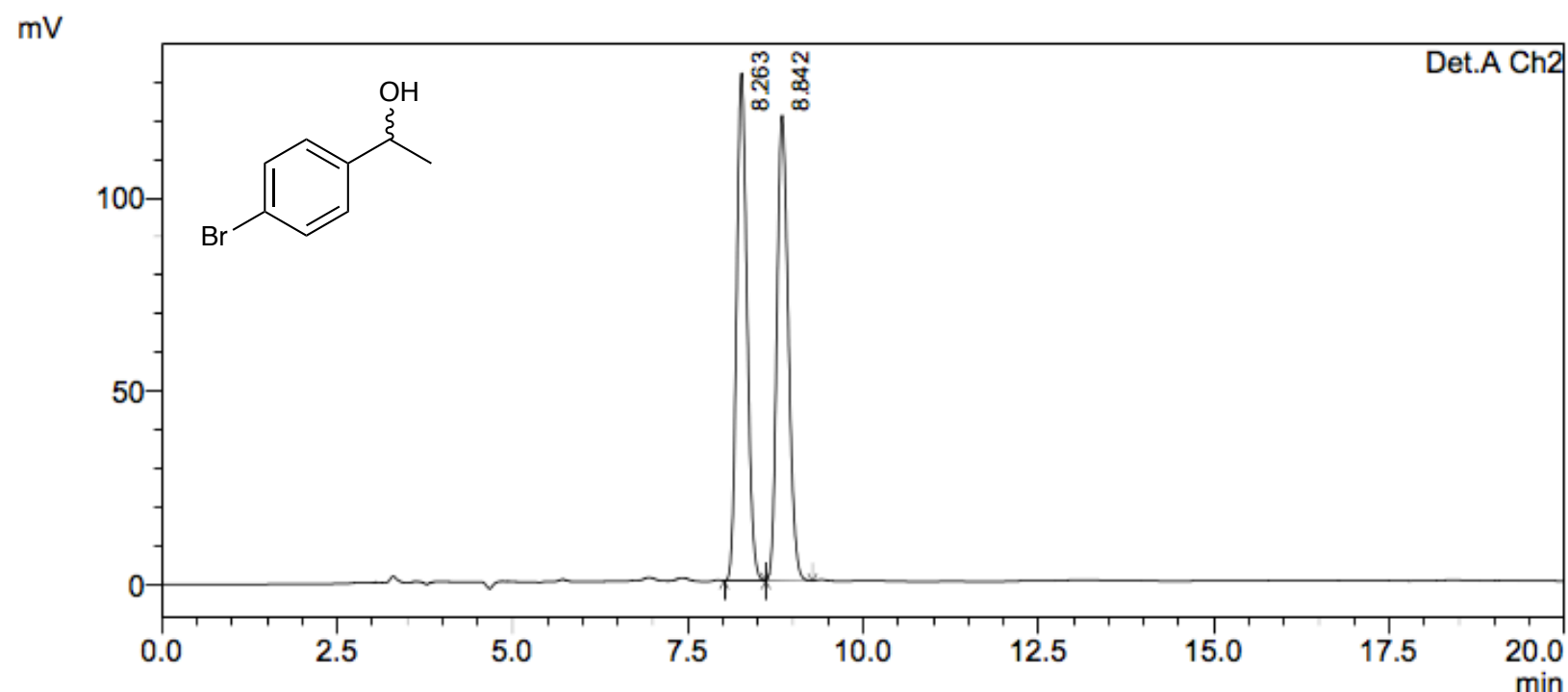

Detector A Ch2 254nm

\begin{tabular}{|r|r|r|r|r|r|}
\hline \multicolumn{1}{|c|}{ Peak\# } & Ret. Time & \multicolumn{1}{c|}{ Area } & Height & \multicolumn{1}{c|}{ Area \% } & \multicolumn{1}{c|}{ Height \% } \\
\hline 1 & 8.263 & 1362743 & 131281 & 49.970 & 52.167 \\
\hline 2 & 8.842 & 1364364 & 120374 & 50.030 & 47.833 \\
\hline Total & & 2727107 & 251655 & 100.000 & 100.000 \\
\hline
\end{tabular}

Figure S14. HPLC chromatogram of the reaction mixture produced by $\mathrm{NaBH}_{4}$ reduction of 4'-bromoacetophenone. 
$\mathrm{mV}$

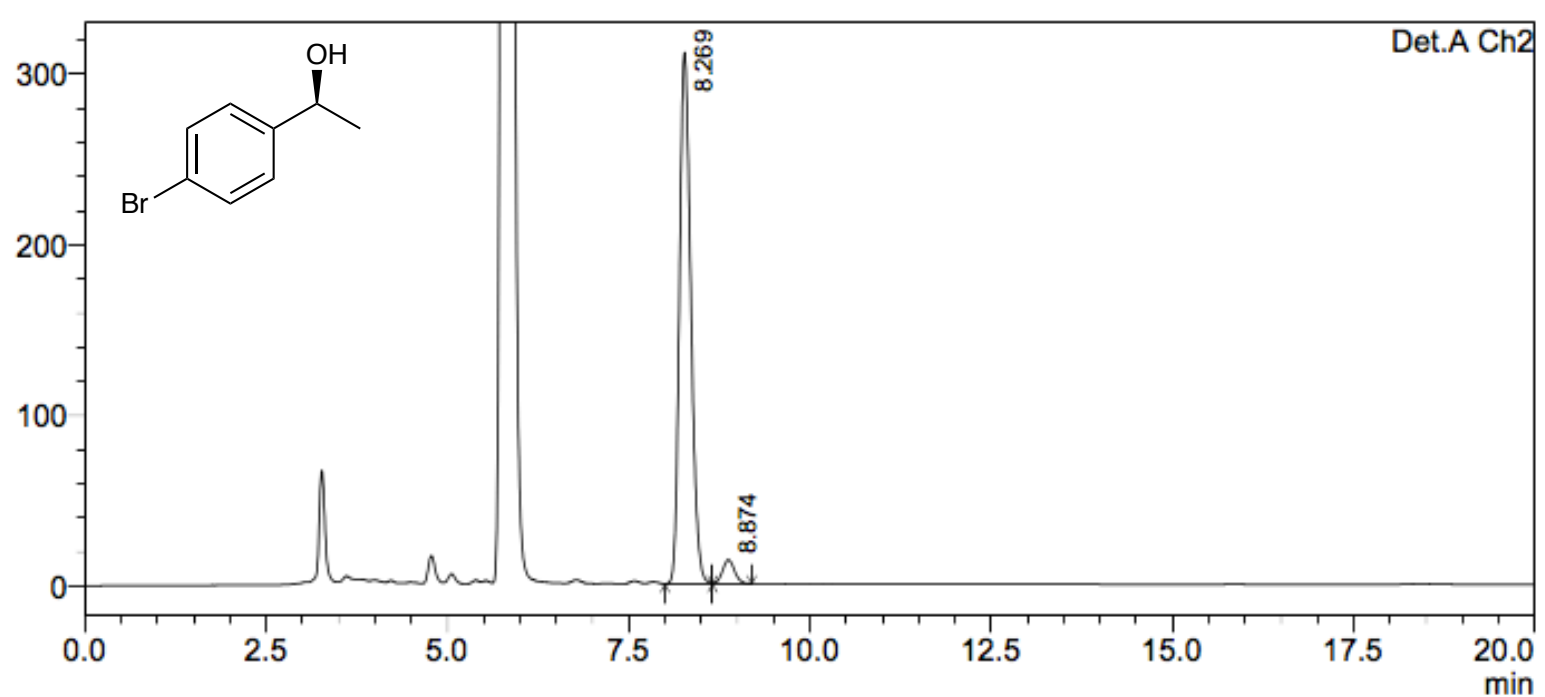

Detector A Ch2 254nm

\begin{tabular}{|r|r|r|r|r|r|}
\hline Peak\# & Ret. Time & \multicolumn{1}{|c|}{ Area } & \multicolumn{1}{c|}{ Height } & Area \% & \multicolumn{1}{c|}{ Height \% } \\
\hline 1 & 8.269 & 3390924 & 311349 & 95.442 & 95.699 \\
\hline 2 & 8.874 & 161958 & 13993 & 4.558 & 4.301 \\
\hline Total & & 3552881 & 325342 & 100.000 & 100.000 \\
\hline
\end{tabular}

Figure S15. HPLC chromatogram of the reaction mixture produced by ATH of 4'bromoacetophenone catalyzed by $\mathrm{Cp}{ }^{*} \operatorname{Ir}\left({ }^{\mathrm{D} P L}-\mathrm{C}_{16}\right)$.

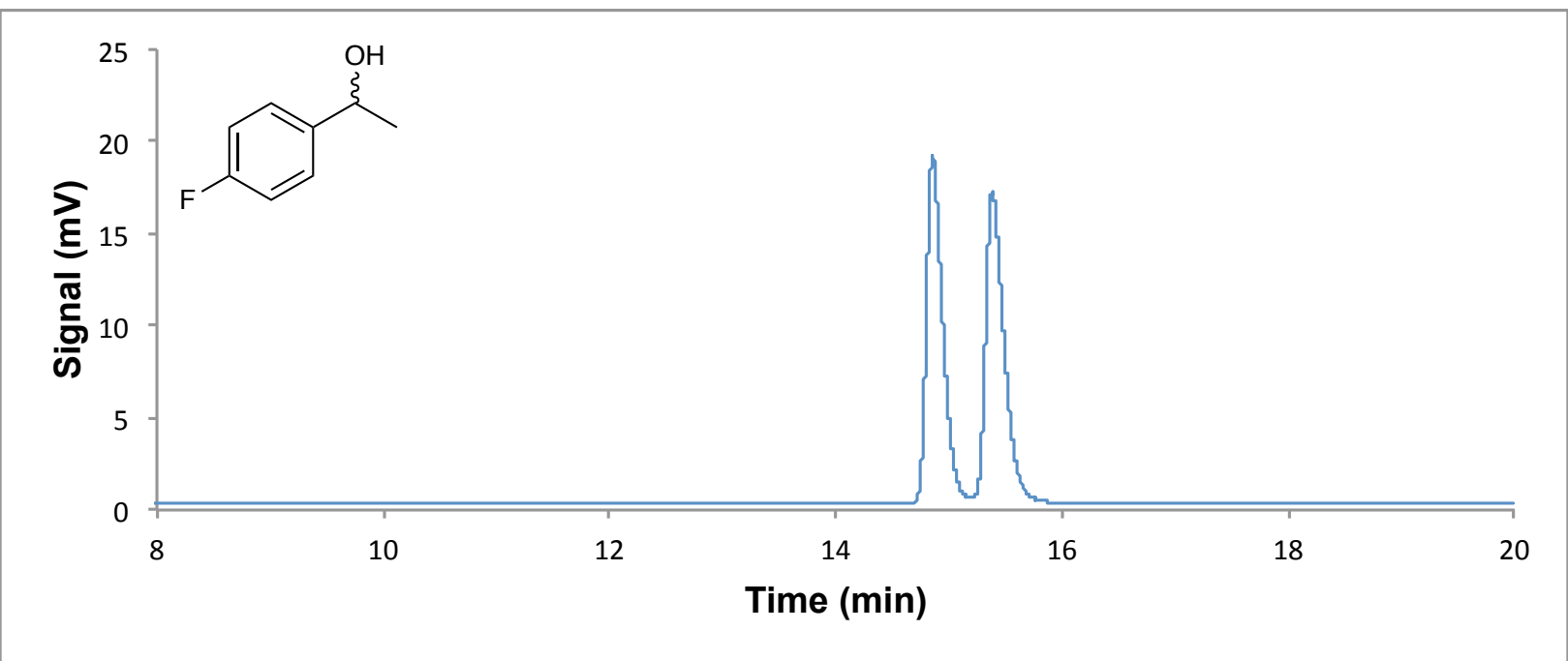

\begin{tabular}{|c|c|c|c|c|c|}
\hline$\#$ & Time & Area & Width & Height & Area \% \\
\hline 1 & 14.857 & 187.9783173 & 18.93641663 & 0.165446937 & 2.710 \\
\hline 2 & 15.385 & 188.8506622 & 16.80952454 & 0.187245682 & 2.713 \\
\hline
\end{tabular}

Figure S16. HPLC chromatogram of the reaction mixture produced by $\mathrm{NaBH}_{4}$ reduction of 4'-fluoroacetophenone. 


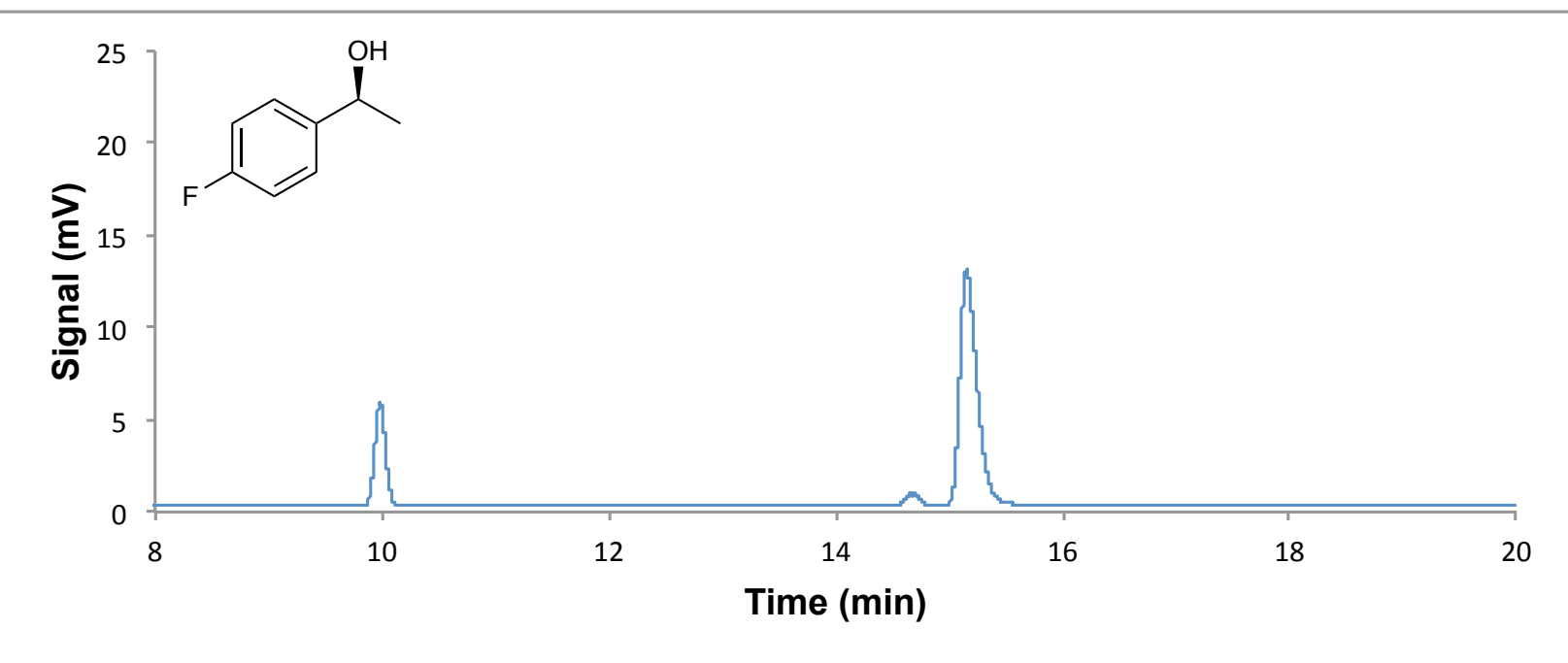

\begin{tabular}{|c|c|c|c|c|c|}
\hline$\#$ & Time & Area & Width & Height & Area \% \\
\hline 1 & 9.984 & 37.60312653 & 5.605824947 & 0.106258452 & 0.463 \\
\hline 2 & 14.669 & 6.31103754 & 0.66513133 & 0.150698096 & 0.078 \\
\hline 3 & 15.155 & 136.2010956 & 12.80424786 & 0.164100438 & 1.678 \\
\hline
\end{tabular}

Figure S17. HPLC chromatogram of the reaction mixture produced by ATH of 4'fluoroacetophenone catalyzed by $C p^{*} \operatorname{Ir}\left({ }^{D} P L-C_{16}\right)$.

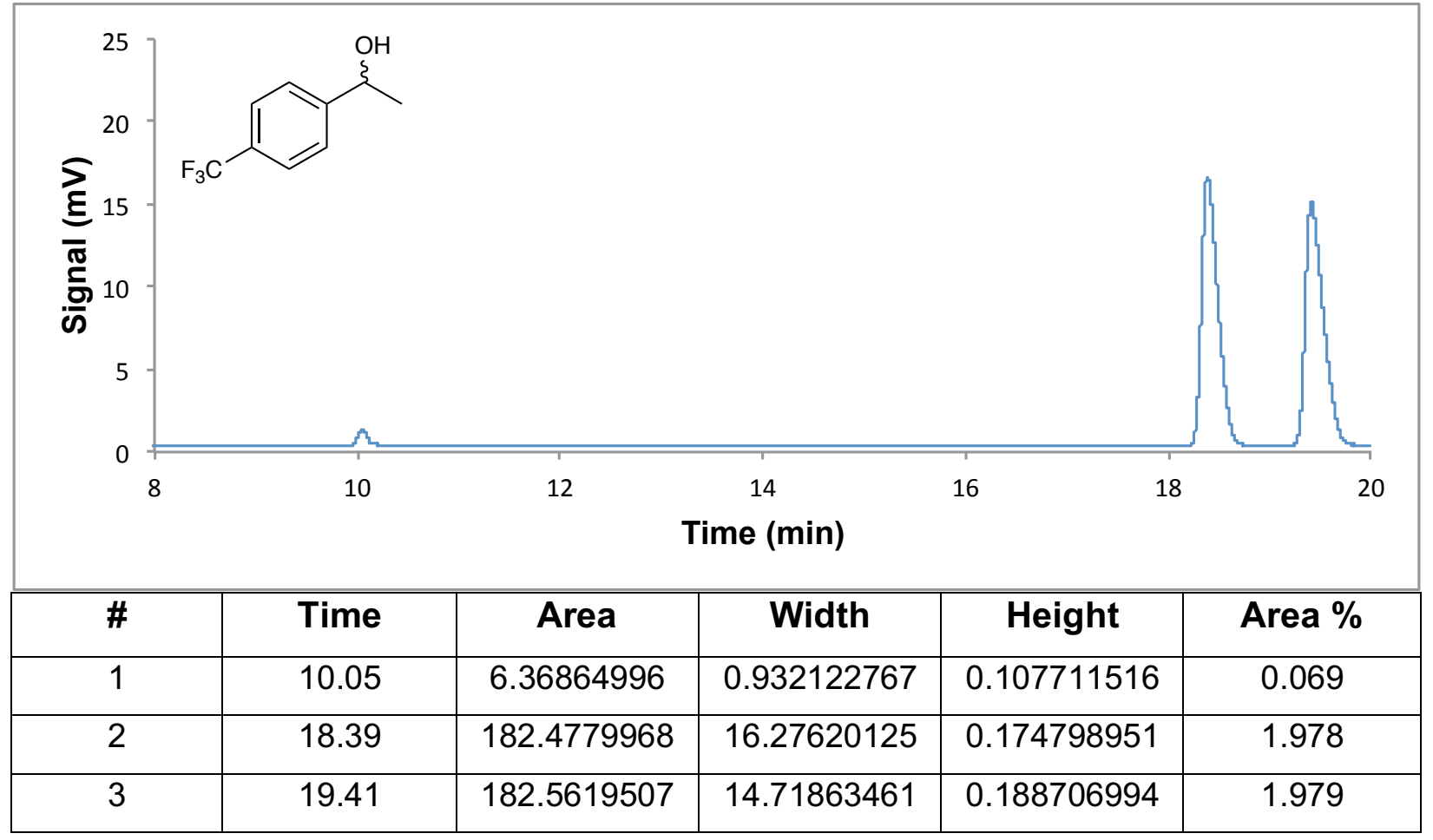

Figure S18. HPLC chromatogram of the reaction mixture produced by $\mathrm{NaBH}_{4}$ reduction of 4'-(trifluoromethyl)acetophenone. 


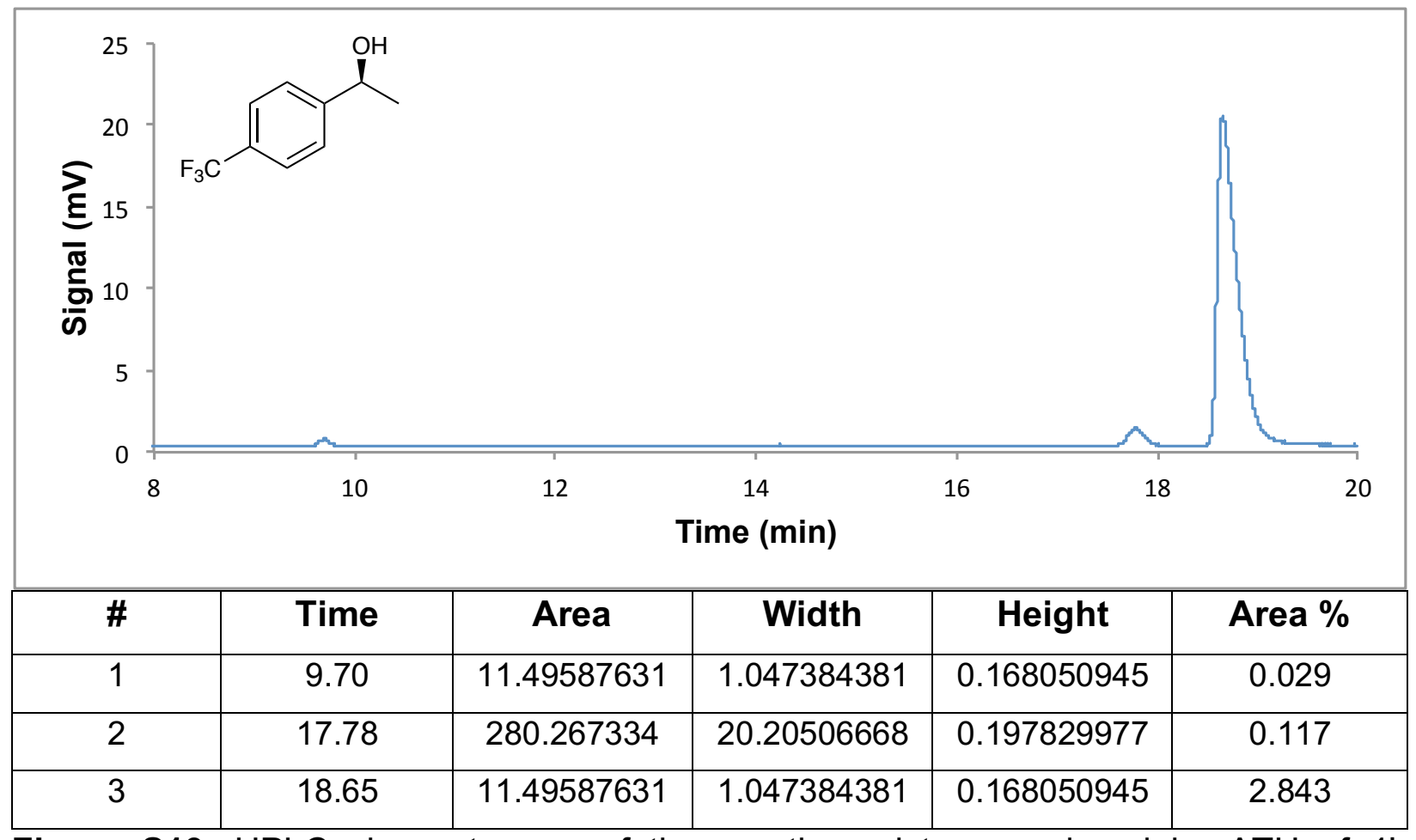

Figure S19. HPLC chromatogram of the reaction mixture produced by ATH of 4'(trifluoromethyl)acetophenone catalyzed by $\mathrm{Cp}{ }^{*} \operatorname{Ir}\left({ }^{\mathrm{D} P L}-\mathrm{C}_{16}\right)$.

$\mathrm{mV}$

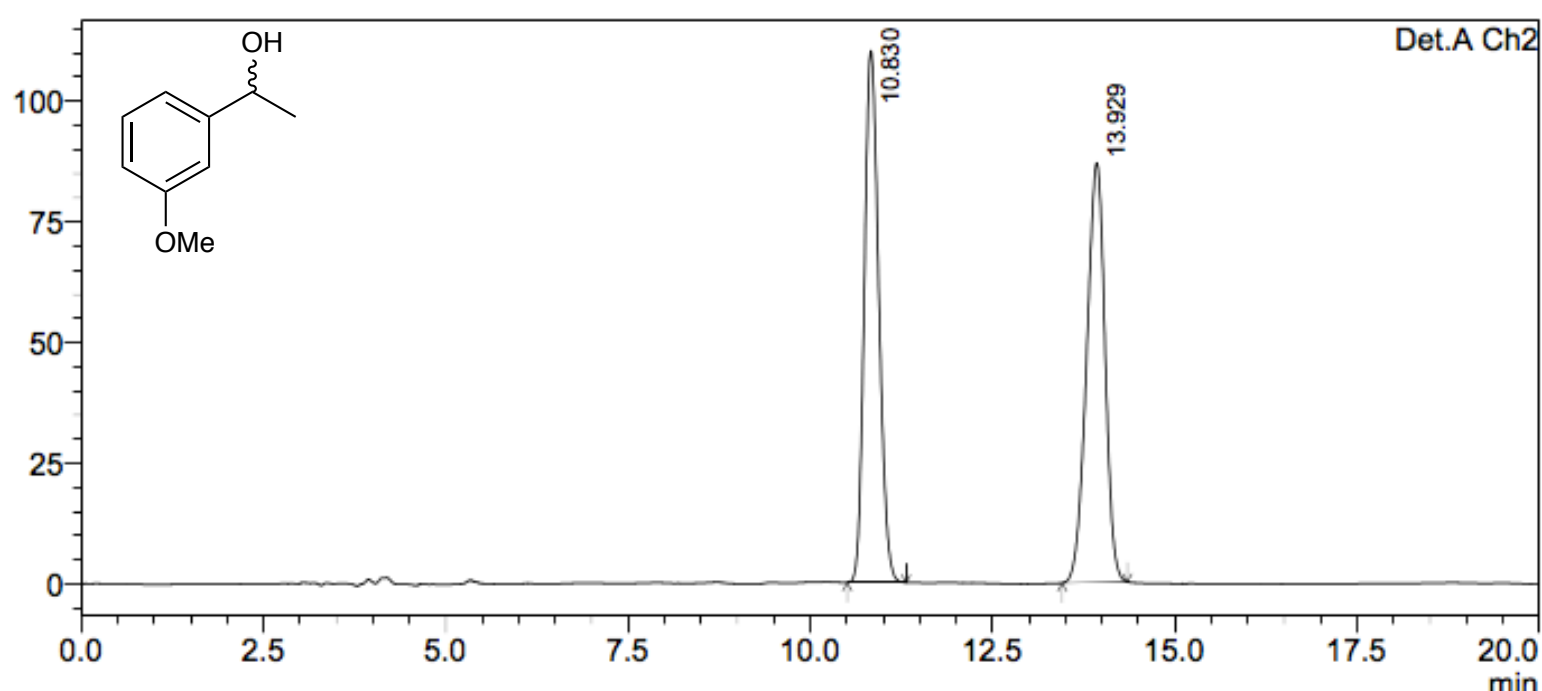

Detector A Ch2 254nm

\begin{tabular}{|r|r|r|r|r|r|}
\hline \multicolumn{1}{|c|}{ Peak\# } & Ret. Time & \multicolumn{1}{c|}{ Area } & Height & \multicolumn{1}{c|}{ Area \% } & \multicolumn{1}{c|}{ Height \% } \\
\hline 1 & 10.830 & 1521456 & 110179 & 49.942 & 55.911 \\
\hline 2 & 13.929 & 1524993 & 86882 & 50.058 & 44.089 \\
\hline Total & & 3046450 & 197060 & 100.000 & 100.000 \\
\hline
\end{tabular}

Figure S20. HPLC chromatogram of the reaction mixture produced by $\mathrm{NaBH}_{4}$ reduction of 3'-methoxyacetophenone. 


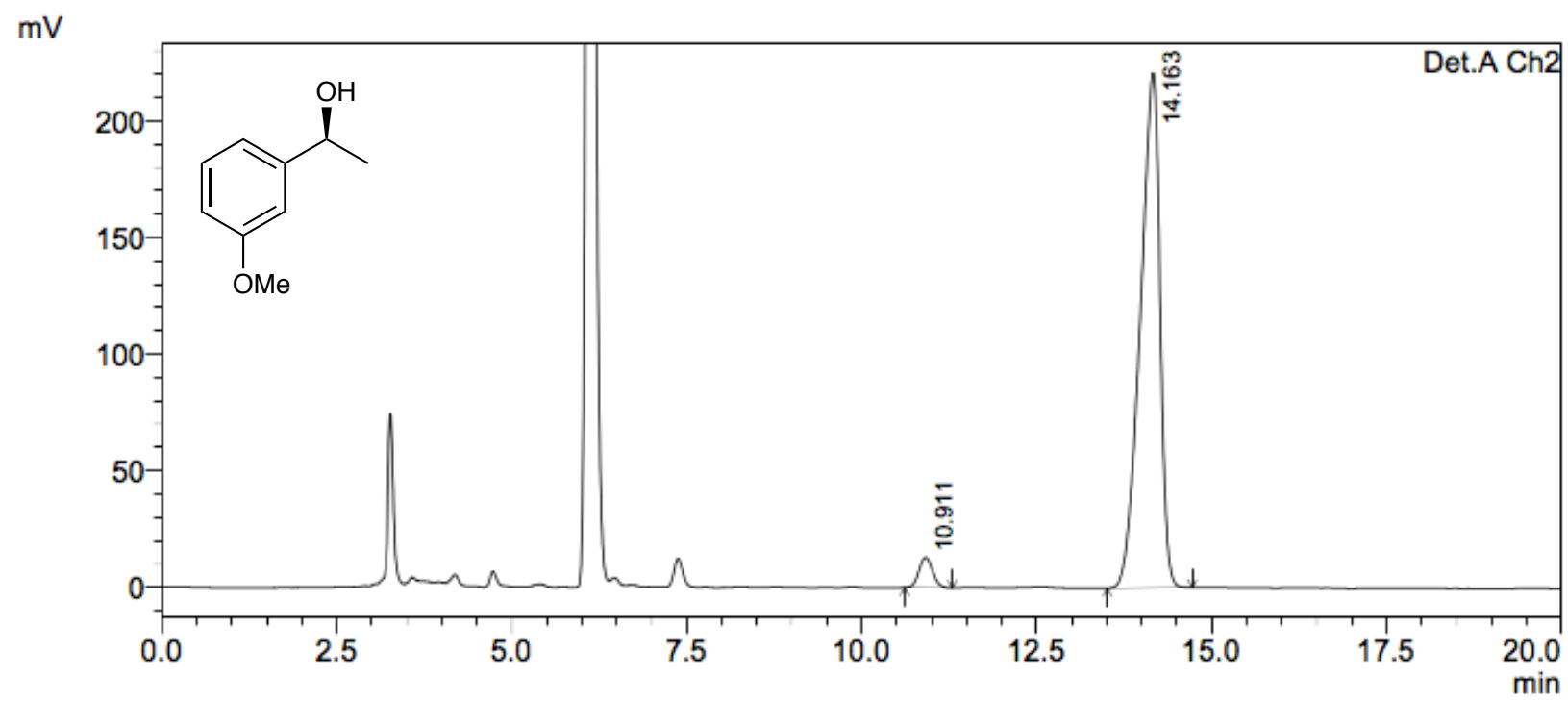

Detector A Ch2 254nm

\begin{tabular}{|r|r|r|r|r|r|}
\hline \multicolumn{1}{|c|}{ Peak\# } & \multicolumn{1}{|c|}{ Ret. Time } & \multicolumn{1}{c|}{ Area } & Height & Area \% & \multicolumn{1}{c|}{ Height \% } \\
\hline 1 & 10.911 & 176068 & 13044 & 3.926 & 5.579 \\
\hline 2 & 14.163 & 4308546 & 220746 & 96.074 & 94.421 \\
\hline Total & & 4484615 & 233790 & 100.000 & 100.000 \\
\hline
\end{tabular}

Figure S21. HPLC chromatogram of the reaction mixture produced by ATH of 3'methoxyacetophenone catalyzed by $\mathrm{Cp}{ }^{*} \operatorname{Ir}\left({ }^{\mathrm{DPL}}-\mathrm{C}_{16}\right)$.

$\mathrm{mV}$

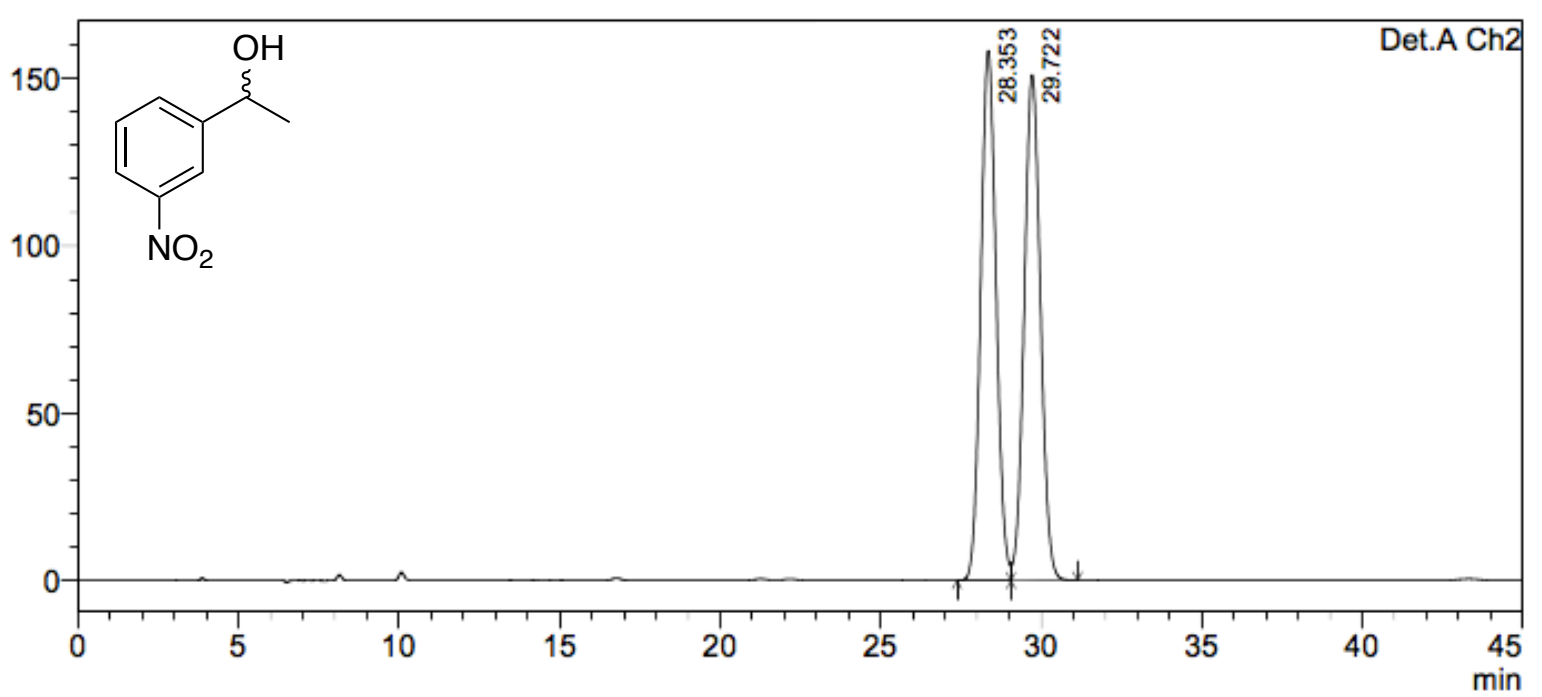

Detector A Ch2 254nm

\begin{tabular}{|r|r|r|r|r|r|}
\hline \multicolumn{1}{|c|}{ Peak\# } & Ret. Time & \multicolumn{1}{c|}{ Area } & \multicolumn{1}{c|}{ Height } & \multicolumn{1}{c|}{ Area \% } & \multicolumn{1}{c|}{ Height \% } \\
\hline 1 & 28.353 & 5289939 & 158174 & 49.908 & 51.183 \\
\hline 2 & 29.722 & 5309393 & 150865 & 50.092 & 48.817 \\
\hline Total & & 10599331 & 309039 & 100.000 & 100.000 \\
\hline
\end{tabular}

Figure S22. HPLC chromatogram of the reaction mixture produced by $\mathrm{NaBH}_{4}$ reduction of 3'-nitroacetophenone. 


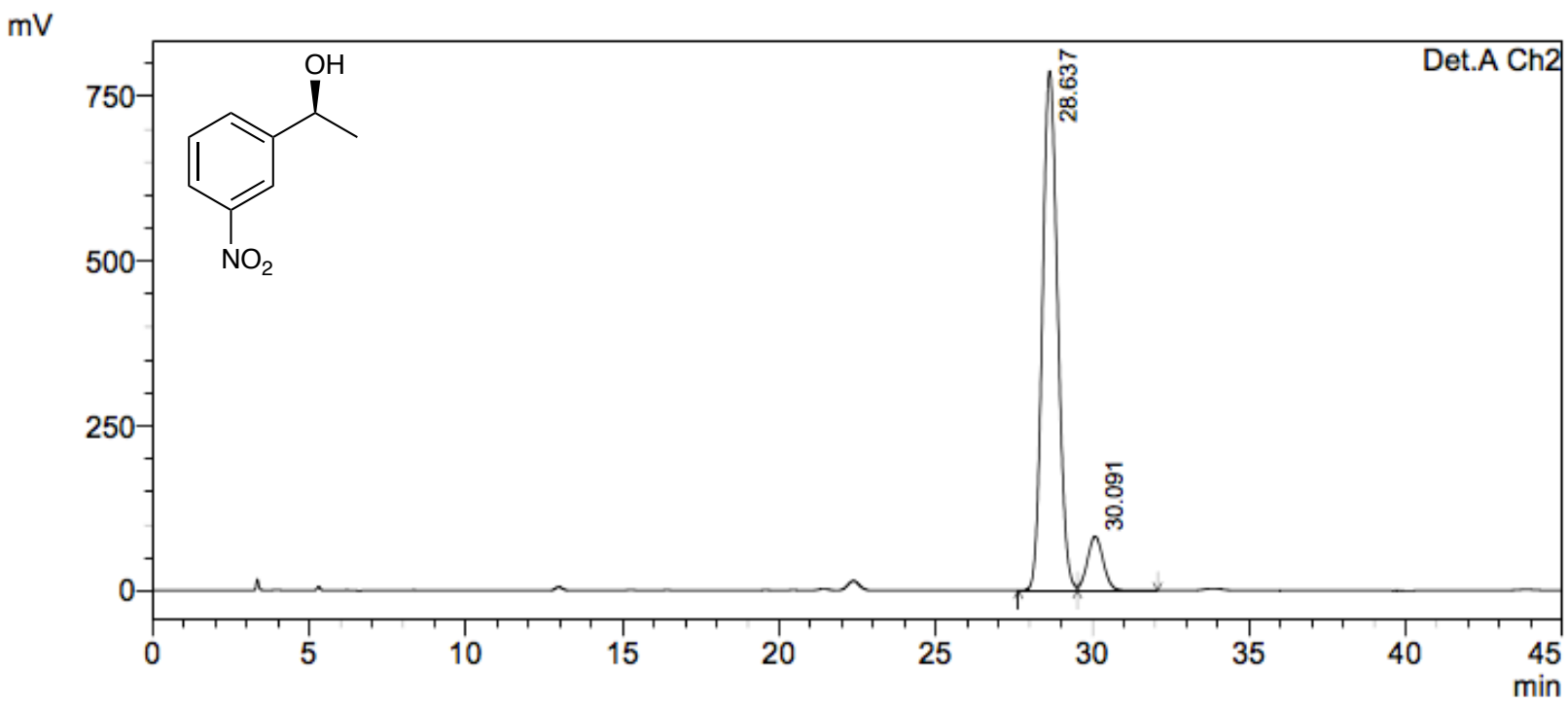

Detector A Ch2 254nm

\begin{tabular}{|r|r|r|r|r|r|}
\hline \multicolumn{1}{|c|}{ Peak\# } & \multicolumn{1}{|c|}{ Ret. Time } & \multicolumn{1}{c|}{ Area } & \multicolumn{1}{c|}{ Height } & \multicolumn{1}{c|}{ Area \% } & \multicolumn{1}{c|}{ Height \% } \\
\hline 1 & 28.637 & 26602800 & 788011 & 90.183 & 90.570 \\
\hline 2 & 30.091 & 2895810 & 82046 & 9.817 & 9.430 \\
\hline Total & & 29498610 & 870057 & 100.000 & 100.000 \\
\hline
\end{tabular}

Figure S23. HPLC chromatogram of the reaction mixture produced by ATH of 3'nitroacetophenone catalyzed by $\mathrm{Cp}{ }^{*} \mathrm{Ir}\left({ }^{\mathrm{DPL}}-\mathrm{C}_{16}\right)$.

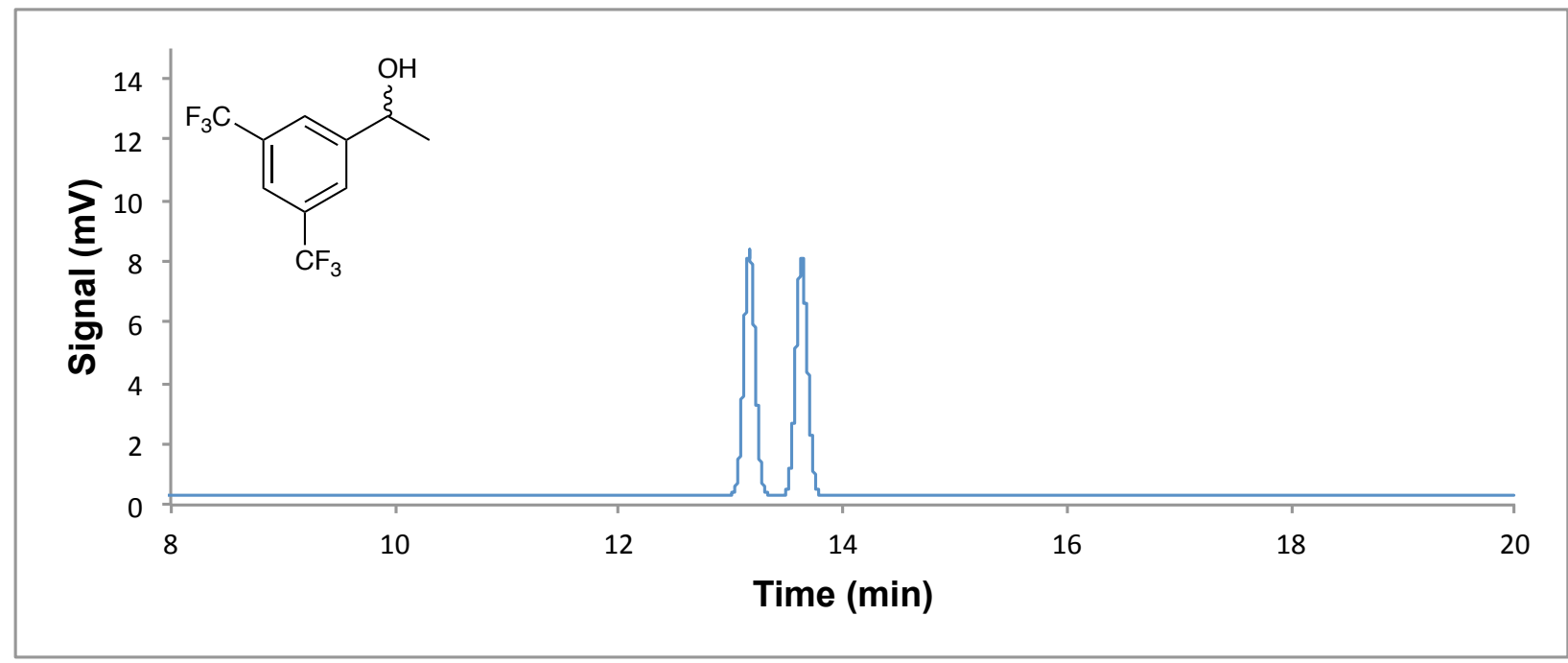

\begin{tabular}{|c|c|c|c|c|c|}
\hline$\#$ & Time & Area & Width & Height & Area \% \\
\hline 1 & 13.166 & 58.52039337 & 8.063076019 & 0.112674631 & 0.644 \\
\hline 2 & 13.631 & 58.59947205 & 7.796162605 & 0.11569193 & 0.645 \\
\hline
\end{tabular}

Figure S24. HPLC chromatogram of the reaction mixture produced by $\mathrm{NaBH}_{4}$ reduction of 3',5'-bis(trifluoromethyl)acetophenone. 


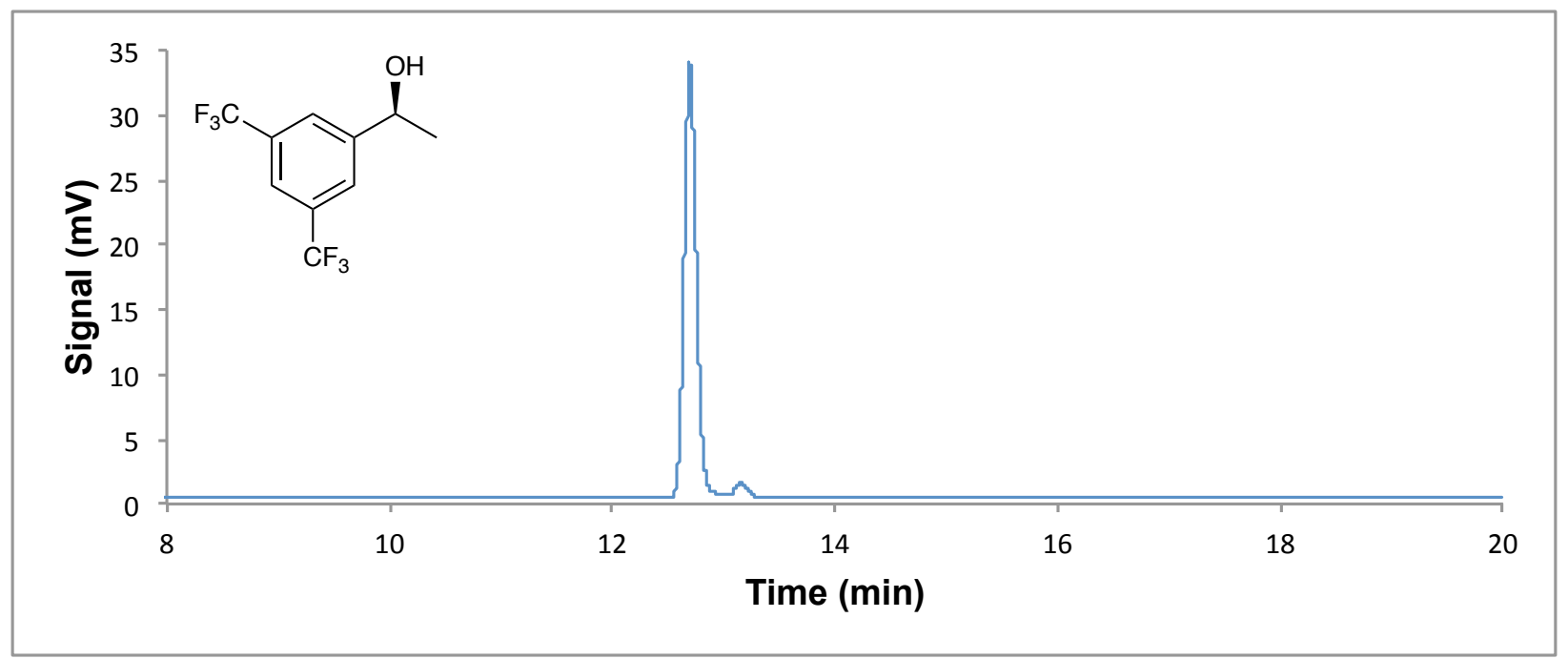

\begin{tabular}{|c|c|c|c|c|c|}
\hline$\#$ & Time & Area & Width & Height & Area \% \\
\hline 1 & 12.698 & 258.4162903 & 33.24071121 & 0.118697718 & 2.655 \\
\hline 2 & 13.159 & 9.677905083 & 1.103026271 & 0.134362832 & 0.099 \\
\hline
\end{tabular}

Figure S25. HPLC chromatogram of the reaction mixture produced by ATH of 3',5'bis(trifluoromethyl)acetophenone catalyzed by $C p * \operatorname{Ir}\left({ }^{\mathrm{P} P L}-\mathrm{C}_{16}\right)$.

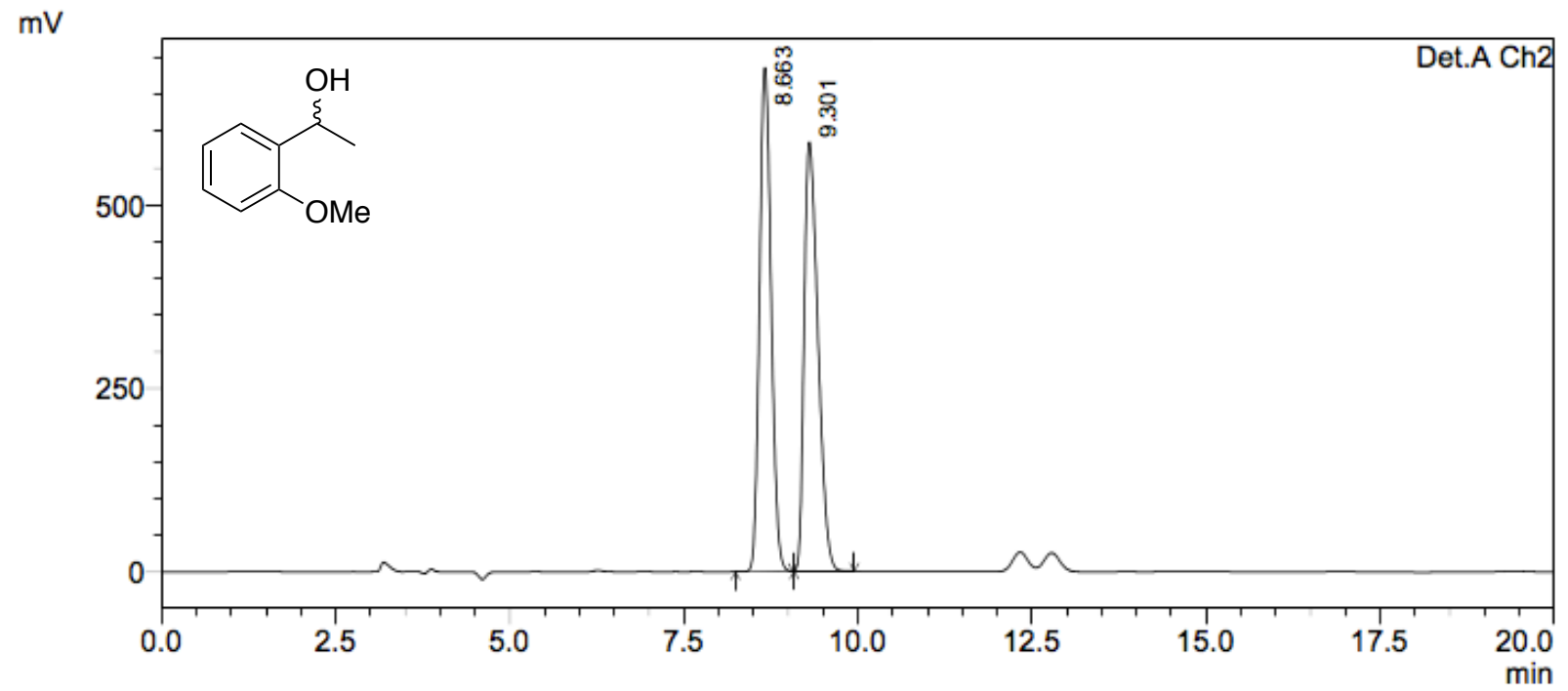

Detector A Ch2 254nm

\begin{tabular}{|r|r|r|r|r|r|}
\hline Peak\# & Ret. Time & \multicolumn{1}{c|}{ Area } & \multicolumn{1}{c|}{ Height } & \multicolumn{1}{c|}{ Area \% } & \multicolumn{1}{c|}{ Height \% } \\
\hline 1 & 8.663 & 7974903 & 685408 & 49.715 & 54.012 \\
\hline 2 & 9.301 & 8066417 & 583584 & 50.285 & 45.988 \\
\hline Total & & 16041320 & 1268992 & 100.000 & 100.000 \\
\hline
\end{tabular}

Figure S26. HPLC chromatogram of the reaction mixture produced by $\mathrm{NaBH}_{4}$ reduction of 2'-methoxyacetophenone. 


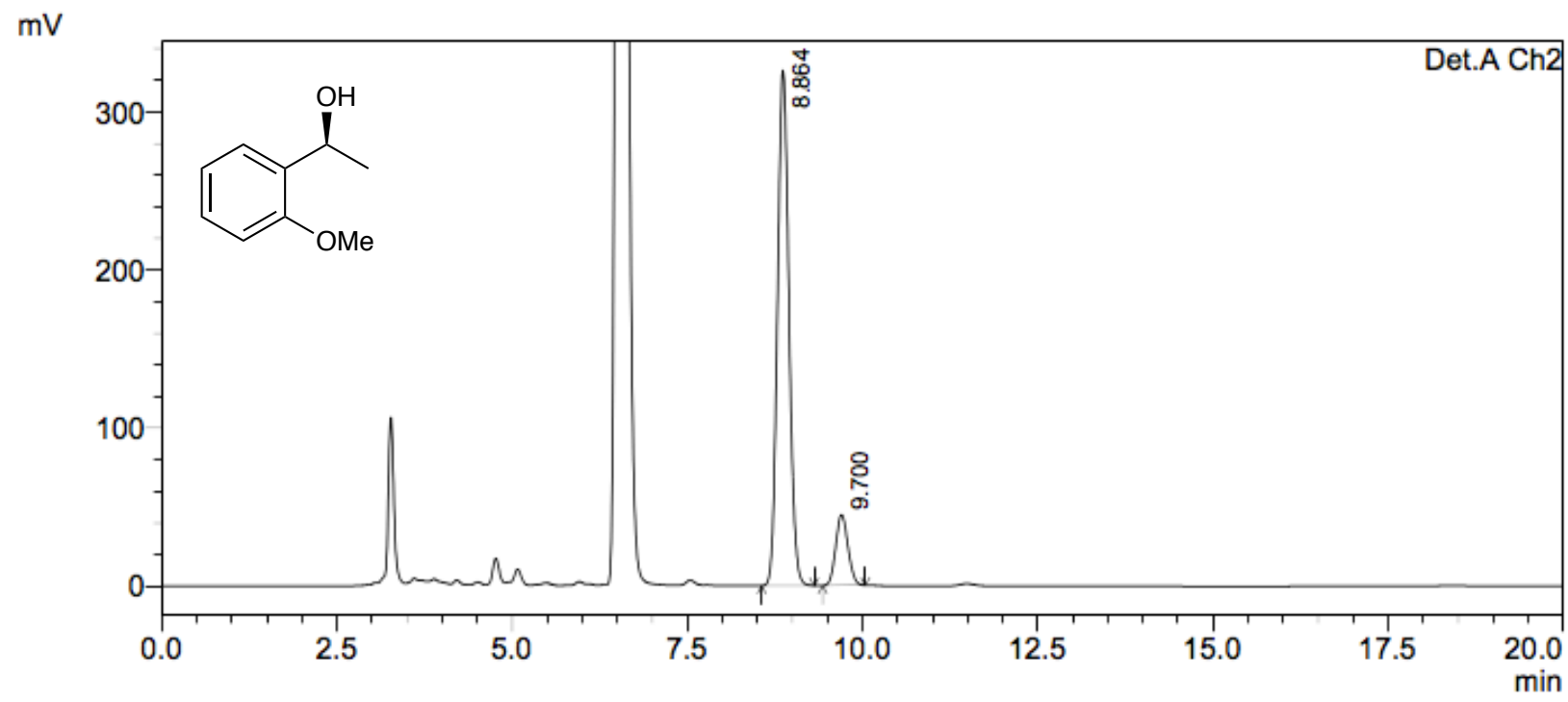

Detector A Ch2 254nm

\begin{tabular}{|r|r|r|r|r|r|}
\hline \multicolumn{1}{|c|}{ Peak\# } & \multicolumn{1}{c|}{ Ret. Time } & \multicolumn{1}{c|}{ Area } & \multicolumn{1}{c|}{ Height } & \multicolumn{1}{c|}{ Area \% } & \multicolumn{1}{c|}{ Height \% } \\
\hline 1 & 8.864 & 3706780 & 325886 & 87.130 & 87.933 \\
\hline 2 & 9.700 & 547534 & 44720 & 12.870 & 12.067 \\
\hline Total & & 4254314 & 370605 & 100.000 & 100.000 \\
\hline
\end{tabular}

Figure S27. HPLC chromatogram of the reaction mixture produced by ATH of 2'methoxyacetophenone catalyzed by $\mathrm{Cp}{ }^{*} \operatorname{Ir}\left({ }^{\mathrm{DPL}}-\mathrm{C}_{16}\right)$.

$\mathrm{mV}$

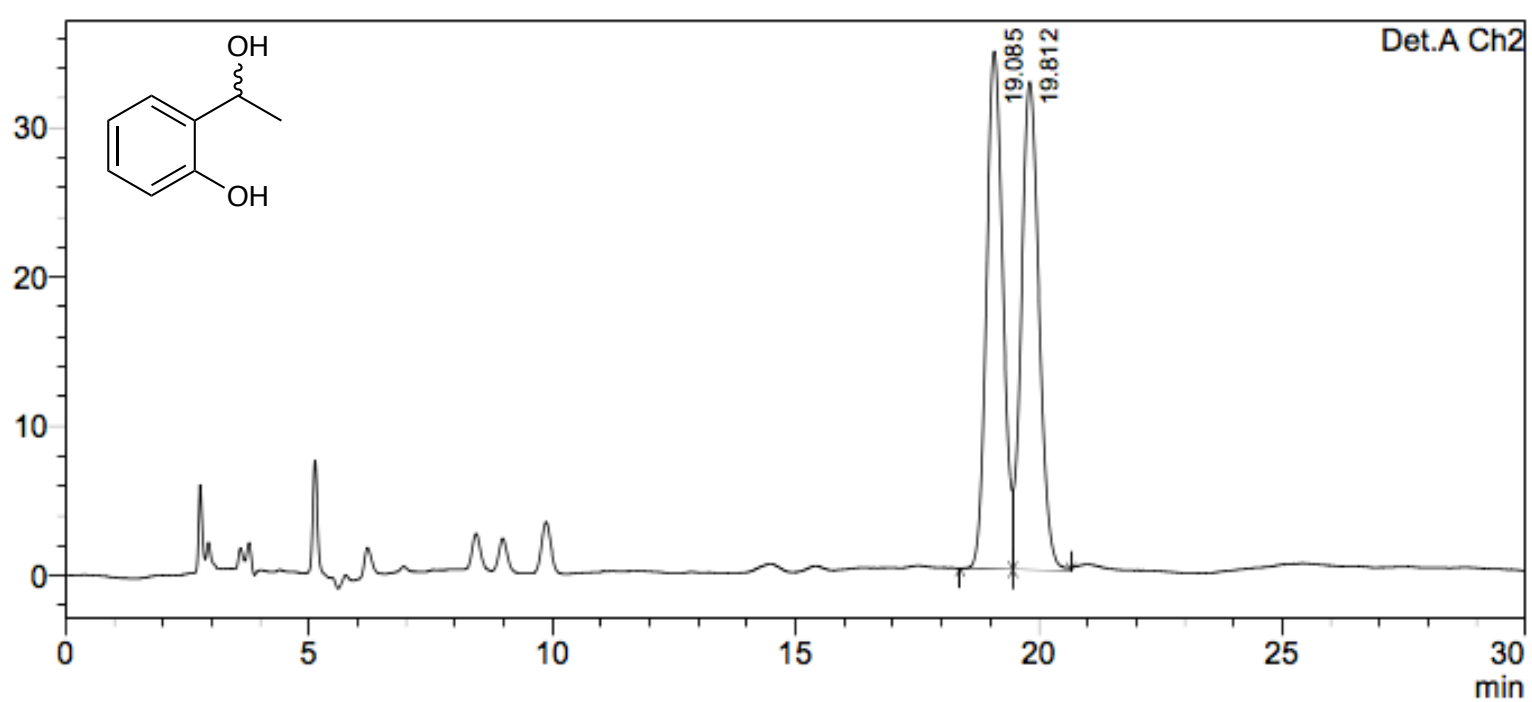

Detector A Ch2 254nm

\begin{tabular}{|r|r|r|r|r|r|}
\hline \multicolumn{1}{|c|}{ Peak\# } & Ret. Time & \multicolumn{1}{c|}{ Area } & \multicolumn{1}{c|}{ Height } & Area \% & Height \% \\
\hline 1 & 19.085 & 810348 & 34679 & 49.499 & 51.475 \\
\hline 2 & 19.812 & 826748 & 32691 & 50.501 & 48.525 \\
\hline Total & & 1637096 & 67370 & 100.000 & 100.000 \\
\hline
\end{tabular}

Figure S28. HPLC chromatogram of the reaction mixture produced by $\mathrm{NaBH}_{4}$ reduction of 2'-hydroxyacetophenone. 


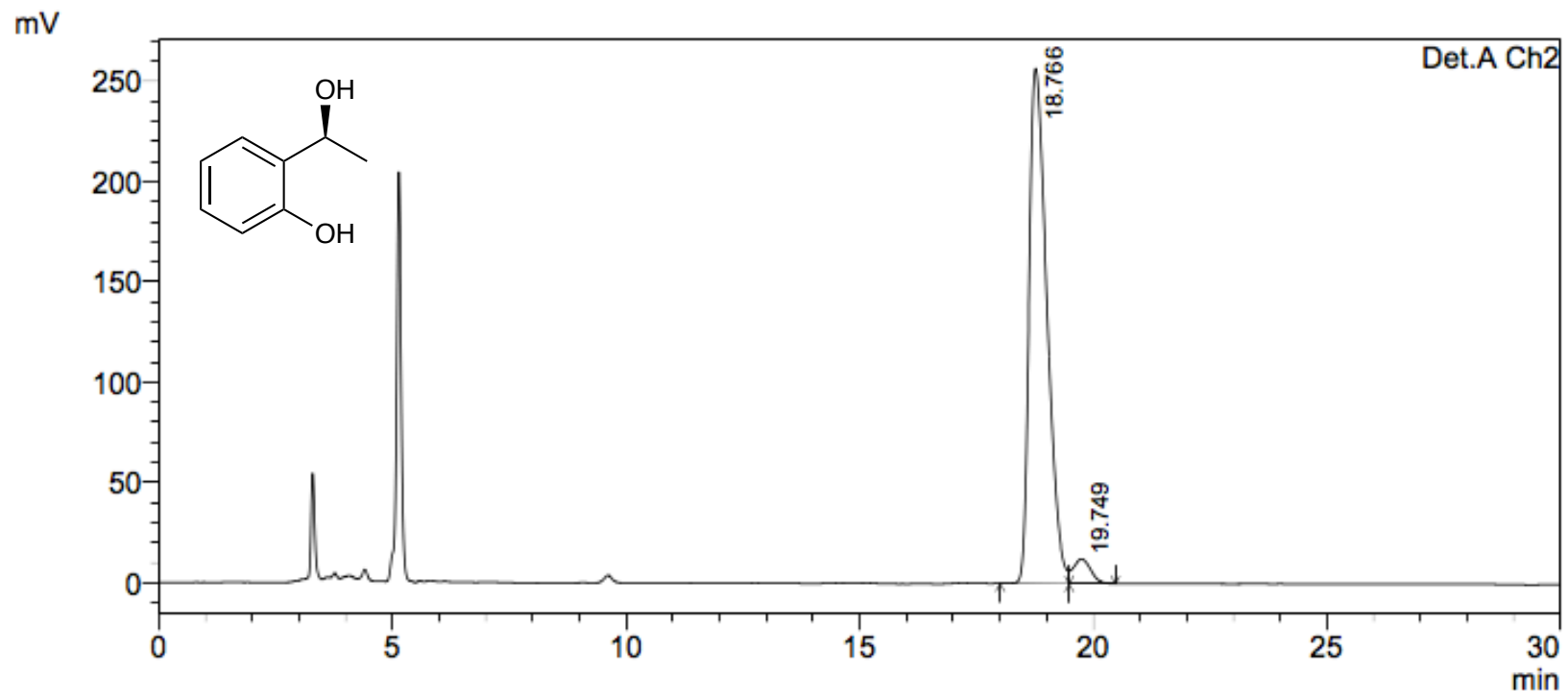

Detector A Ch2 254nm

\begin{tabular}{|r|r|r|r|r|r|}
\hline \multicolumn{1}{|c|}{ Peak\# } & \multicolumn{1}{c|}{ Ret. Time } & \multicolumn{1}{c|}{ Area } & \multicolumn{1}{c|}{ Height } & \multicolumn{1}{c|}{ Area \% } & \multicolumn{1}{c|}{ Height \% } \\
\hline 1 & 18.766 & 6783218 & 256731 & 95.485 & 95.452 \\
\hline 2 & 19.749 & 320744 & 12233 & 4.515 & 4.548 \\
\hline Total & & 7103962 & 268965 & 100.000 & 100.000 \\
\hline
\end{tabular}

Figure S29. HPLC chromatogram of the reaction mixture produced by ATH of 2'hydroxyacetophenone catalyzed by $\mathrm{Cp}^{*} \operatorname{Ir}\left({ }^{\mathrm{DPL}}-\mathrm{C}_{16}\right)$.

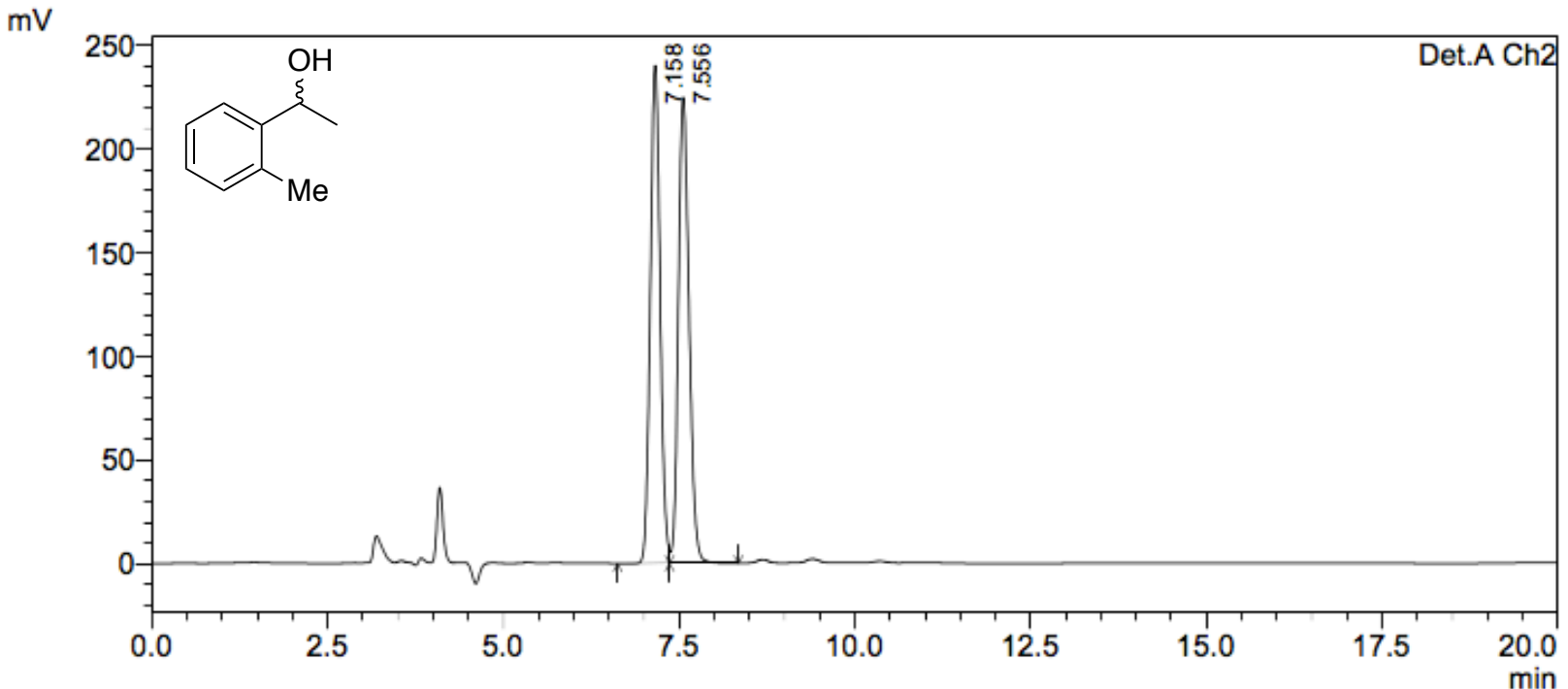

Detector A Ch2 254nm

\begin{tabular}{|r|r|r|r|r|r|}
\hline \multicolumn{1}{|c|}{ Peak\# } & Ret. Time & \multicolumn{1}{c|}{ Area } & Height & Area \% & \multicolumn{1}{c|}{ Height \% } \\
\hline 1 & 7.158 & 2342874 & 239947 & 49.692 & 51.654 \\
\hline 2 & 7.556 & 2371878 & 224578 & 50.308 & 48.346 \\
\hline Total & & 4714752 & 464525 & 100.000 & 100.000 \\
\hline
\end{tabular}

Figure S30. HPLC chromatogram of the reaction mixture produced by $\mathrm{NaBH}_{4}$ reduction of 2'-methoxyacetophenone. 


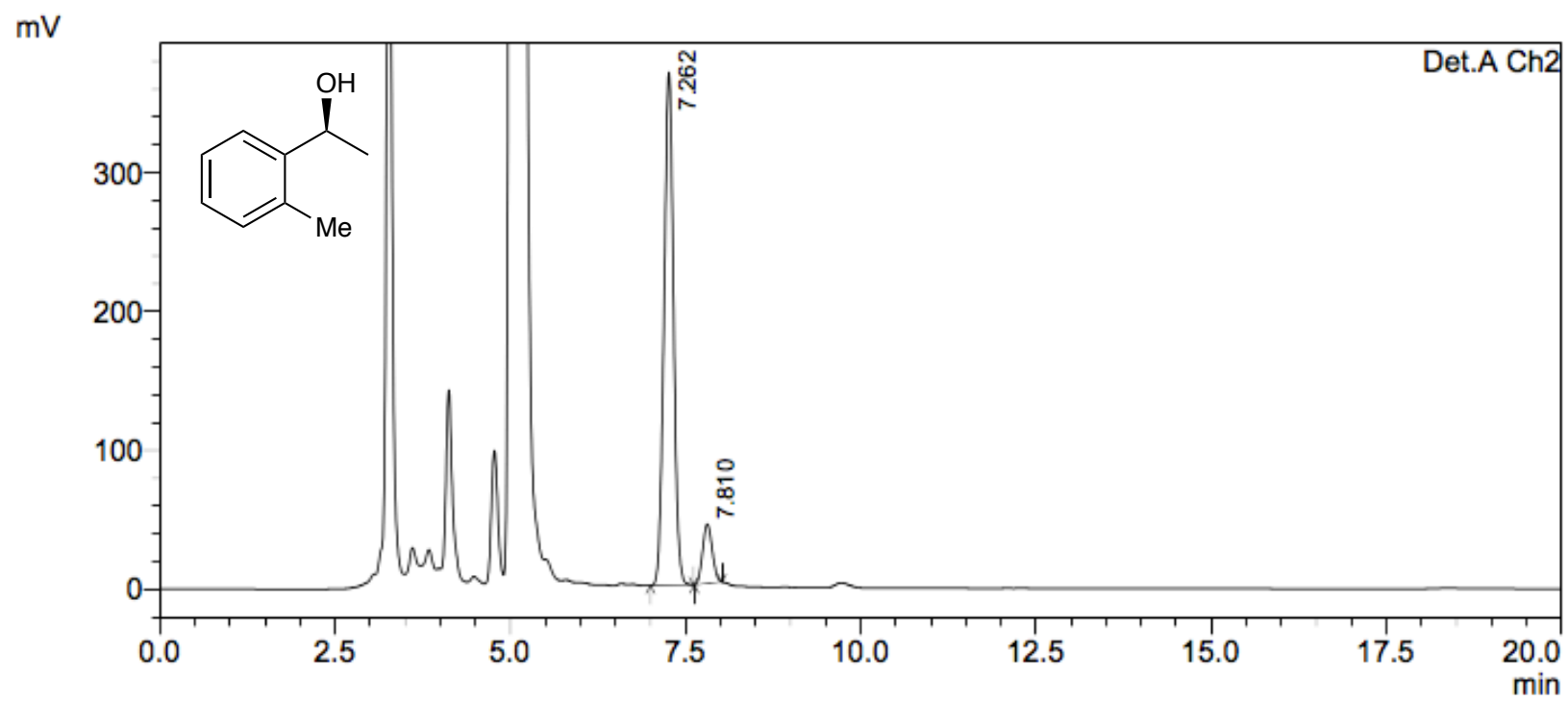

Detector A Ch2 254nm

\begin{tabular}{|r|r|r|r|r|r|}
\hline \multicolumn{1}{|c|}{ Peak\# } & \multicolumn{1}{|c|}{ Ret. Time } & \multicolumn{1}{c|}{ Area } & \multicolumn{1}{c|}{ Height } & \multicolumn{1}{c|}{ Area \% } & \multicolumn{1}{c|}{ Height \% } \\
\hline 1 & 7.262 & 3479315 & 369192 & 89.587 & 89.613 \\
\hline 2 & 7.810 & 404394 & 42793 & 10.413 & 10.387 \\
\hline Total & & 3883709 & 411985 & 100.000 & 100.000 \\
\hline
\end{tabular}

Figure S31. HPLC chromatogram of the reaction mixture produced by ATH of 2'methoxyacetophenone catalyzed by $\mathrm{Cp}{ }^{*} \operatorname{Ir}\left({ }^{\mathrm{D} P L}-\mathrm{C}_{16}\right)$.

\begin{tabular}{|c|c|c|c|c|c|}
\hline & \multicolumn{2}{|c|}{ Area } \\
\hline
\end{tabular}

Figure S32. HPLC chromatogram of the reaction mixture produced by $\mathrm{NaBH}_{4}$ reduction of 2'-chloroacetophenone. 


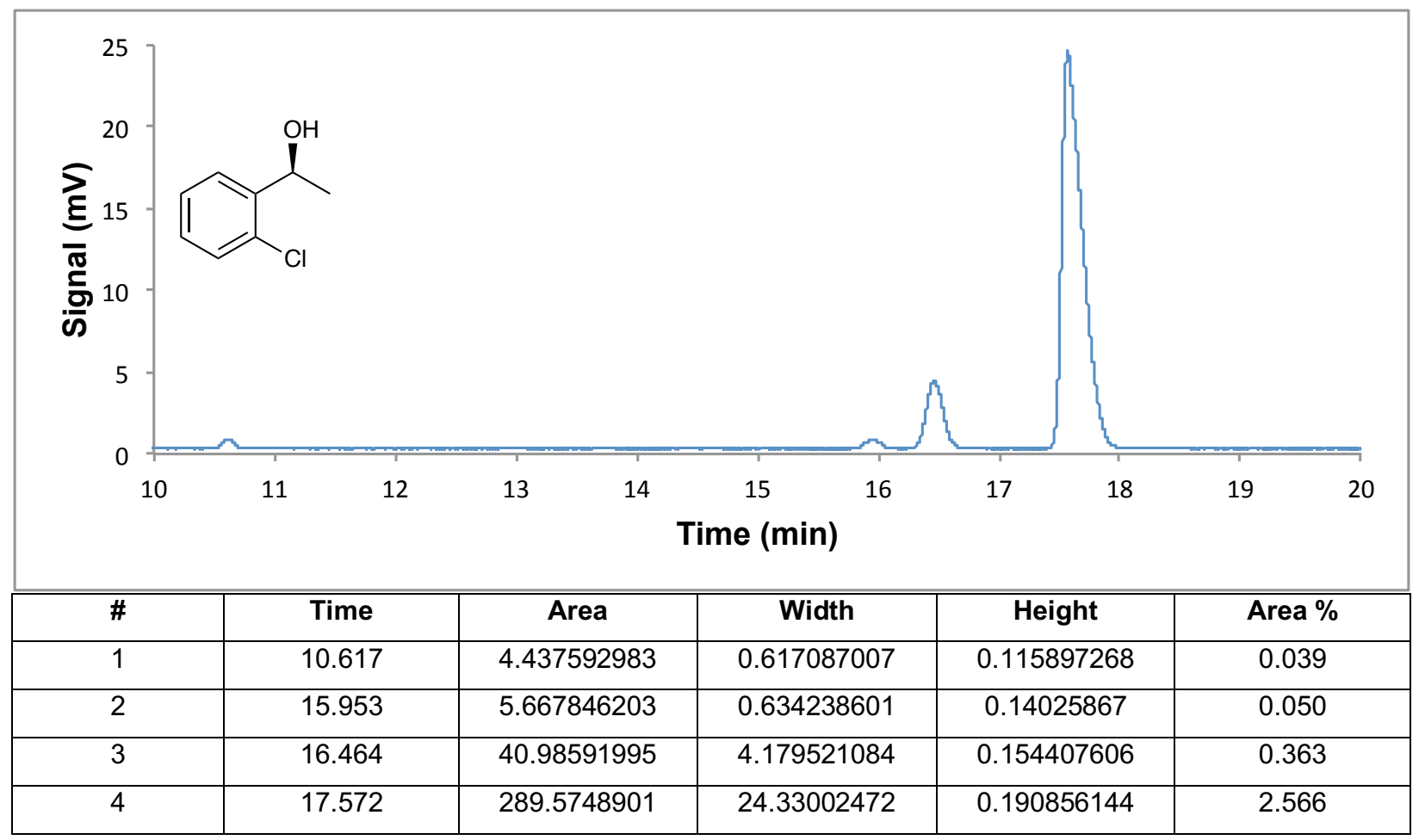

Figure S33. HPLC chromatogram of the reaction mixture produced by ATH of 2'chloroacetophenone catalyzed by $\mathrm{Cp}{ }^{*} \operatorname{Ir}\left({ }^{\mathrm{DPL}}-\mathrm{C}_{16}\right)$.

$\mathrm{mV}$

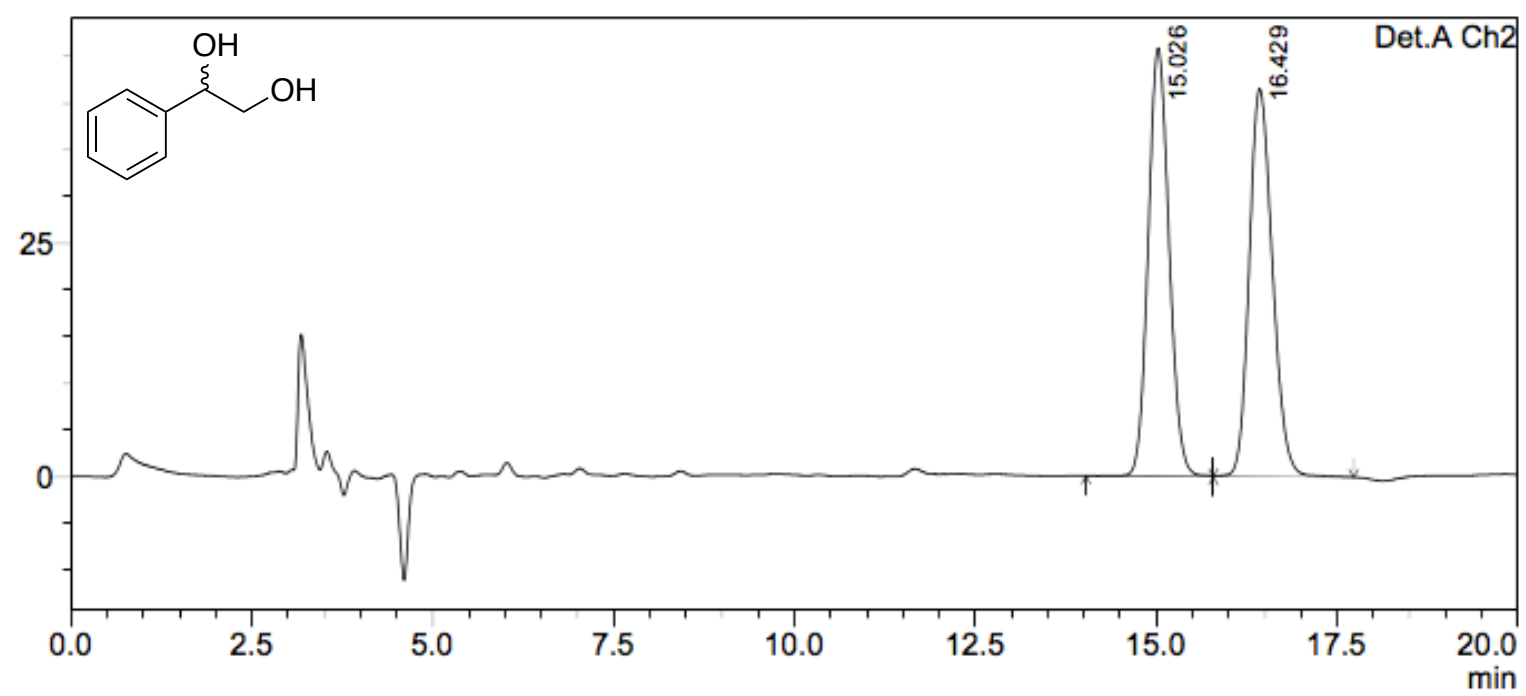

Detector A Ch2 254nm

\begin{tabular}{r|r|r|r|r|r|}
\hline \multicolumn{1}{|c|}{ Peak\# } & Ret. Time & \multicolumn{1}{c|}{ Area } & \multicolumn{1}{c|}{ Height } & \multicolumn{1}{c|}{ Area \% } & \multicolumn{1}{c|}{ Height \% } \\
\hline 1 & 15.026 & 917795 & 45805 & 49.728 & 52.443 \\
\hline 2 & 16.429 & 927826 & 41538 & 50.272 & 47.557 \\
\hline Total & & 1845621 & 87343 & 100.000 & 100.000 \\
\hline
\end{tabular}

Figure S34. HPLC chromatogram of the reaction mixture produced by $\mathrm{NaBH}_{4}$ reduction of 2-hydroxyacetophenone. 


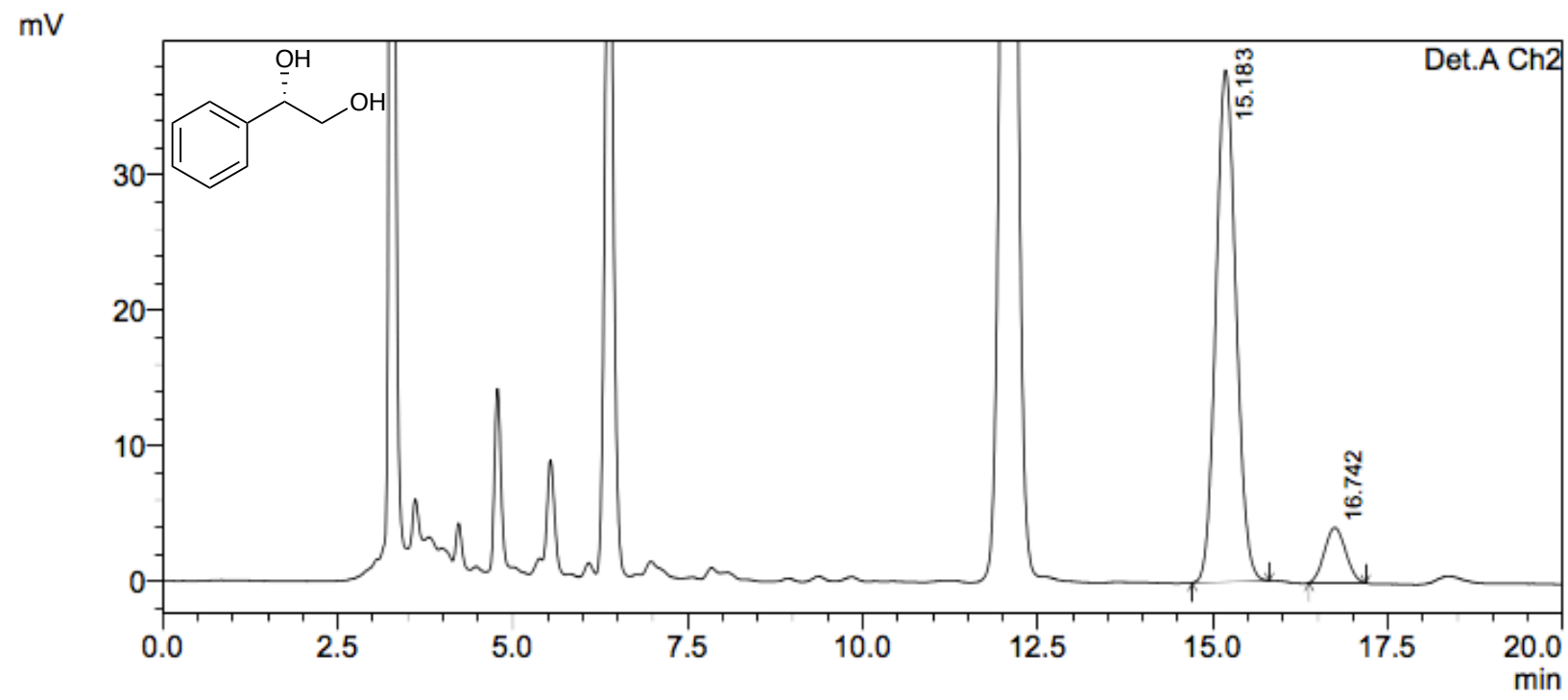

Detector A Ch2 254nm

\begin{tabular}{|r|r|r|r|r|r|}
\hline \multicolumn{1}{|c|}{ Peak\# } & \multicolumn{1}{c|}{ Ret. Time } & \multicolumn{1}{c|}{ Area } & \multicolumn{1}{c|}{ Height } & \multicolumn{1}{c|}{ Area \% } & \multicolumn{1}{c|}{ Height \% } \\
\hline 1 & 15.183 & 754850 & 37778 & 89.772 & 90.264 \\
\hline 2 & 16.742 & 86006 & 4075 & 10.228 & 9.736 \\
\hline Total & & 840855 & 41853 & 100.000 & 100.000 \\
\hline
\end{tabular}

Figure S35. HPLC chromatogram of the reaction mixture produced by ATH of 2hydroxyacetophenone catalyzed by $\mathrm{Cp}{ }^{*} \operatorname{Ir}\left(\mathrm{DPL}^{\left.-\mathrm{C}_{16}\right)}\right.$.

$\mathrm{mV}$

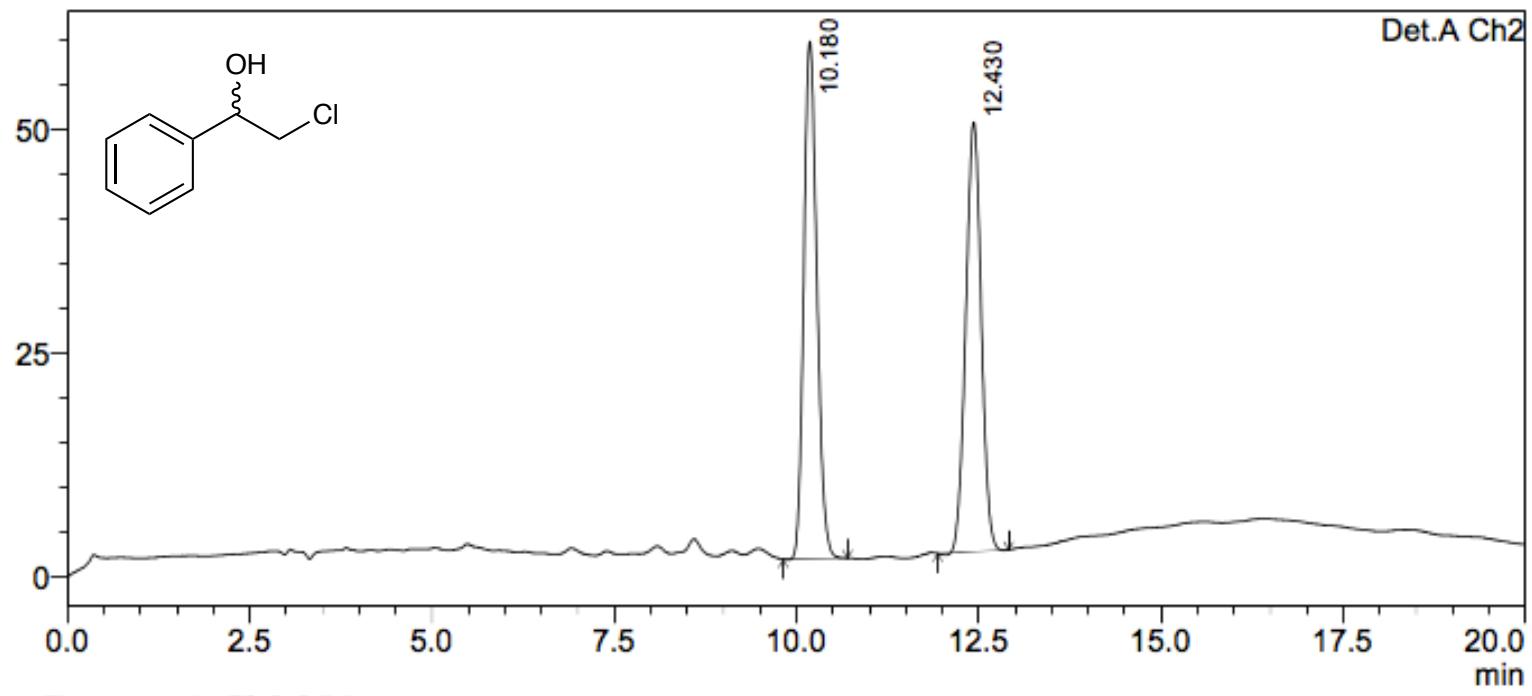

Detector A Ch2 254nm

\begin{tabular}{|r|r|r|r|r|r|}
\hline \multicolumn{1}{|c|}{ Peak\# } & Ret. Time & \multicolumn{1}{c|}{ Area } & \multicolumn{1}{c|}{ Height } & \multicolumn{1}{c|}{ Area \% } & Height \% \\
\hline 1 & 10.180 & 720750 & 57804 & 50.534 & 54.637 \\
\hline 2 & 12.430 & 705511 & 47994 & 49.466 & 45.363 \\
\hline Total & & 1426261 & 105798 & 100.000 & 100.000 \\
\hline
\end{tabular}

Figure S36. HPLC chromatogram of the reaction mixture produced by $\mathrm{NaBH}_{4}$ reduction of 2-chloroacetophenone. 


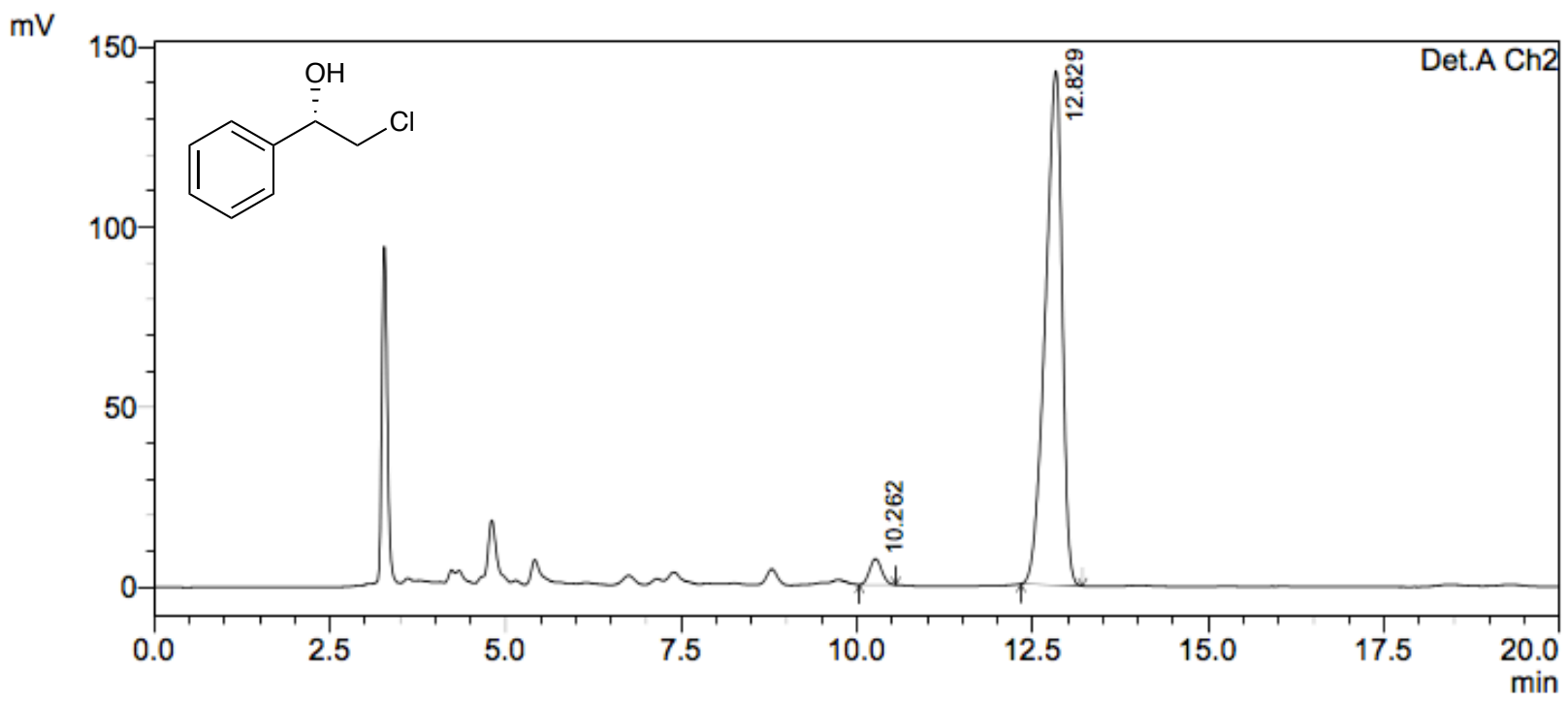

Detector A Ch2 254nm

\begin{tabular}{|r|r|r|r|r|r|}
\hline \multicolumn{1}{|c|}{ Peak\# } & \multicolumn{1}{c|}{ Ret. Time } & \multicolumn{1}{c|}{ Area } & \multicolumn{1}{c|}{ Height } & \multicolumn{1}{c|}{ Area \% } & \multicolumn{1}{c|}{ Height \% } \\
\hline 1 & 10.262 & 86612 & 7204 & 3.517 & 4.806 \\
\hline 2 & 12.829 & 2376137 & 142675 & 96.483 & 95.194 \\
\hline Total & & 2462749 & 149879 & 100.000 & 100.000 \\
\hline
\end{tabular}

Figure S37. HPLC chromatogram of the reaction mixture produced by ATH of 2chloroacetophenone catalyzed by $\mathrm{Cp} * \operatorname{lr}\left({ }^{(} \mathrm{PL}-\mathrm{C}_{16}\right)$.

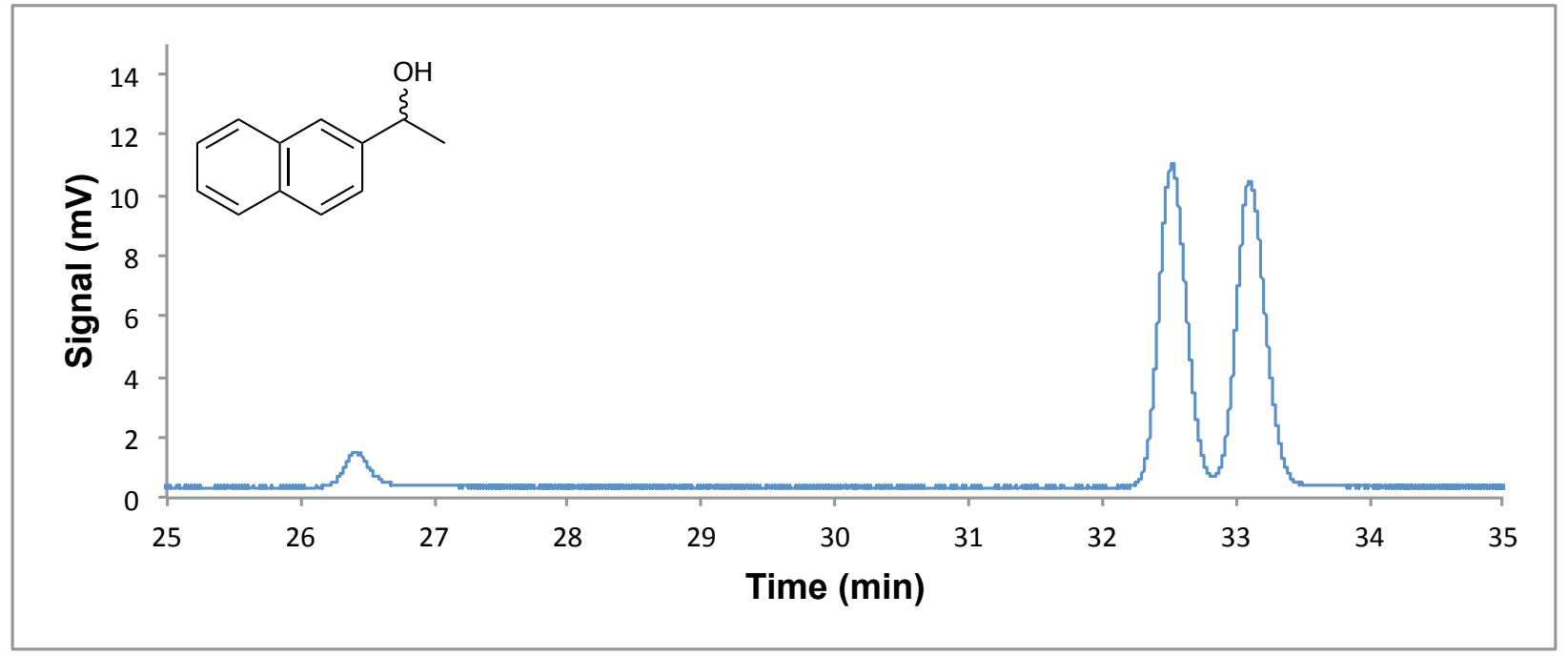

\begin{tabular}{|c|c|c|c|c|c|}
\hline$\#$ & Time & Area & Width & Height & Area \% \\
\hline 1 & 26.418 & 14.9824295 & 1.130485892 & 0.2208848 & 0.181 \\
\hline 2 & 32.524 & 153.3234253 & 10.65937138 & 0.239731804 & 1.856 \\
\hline 3 & 33.098 & 153.7670441 & 10.12266731 & 0.253172785 & 1.861 \\
\hline
\end{tabular}

Figure S38. HPLC chromatogram of the reaction mixture produced by $\mathrm{NaBH}_{4}$ reduction of 2-acetylnaphthalene. 


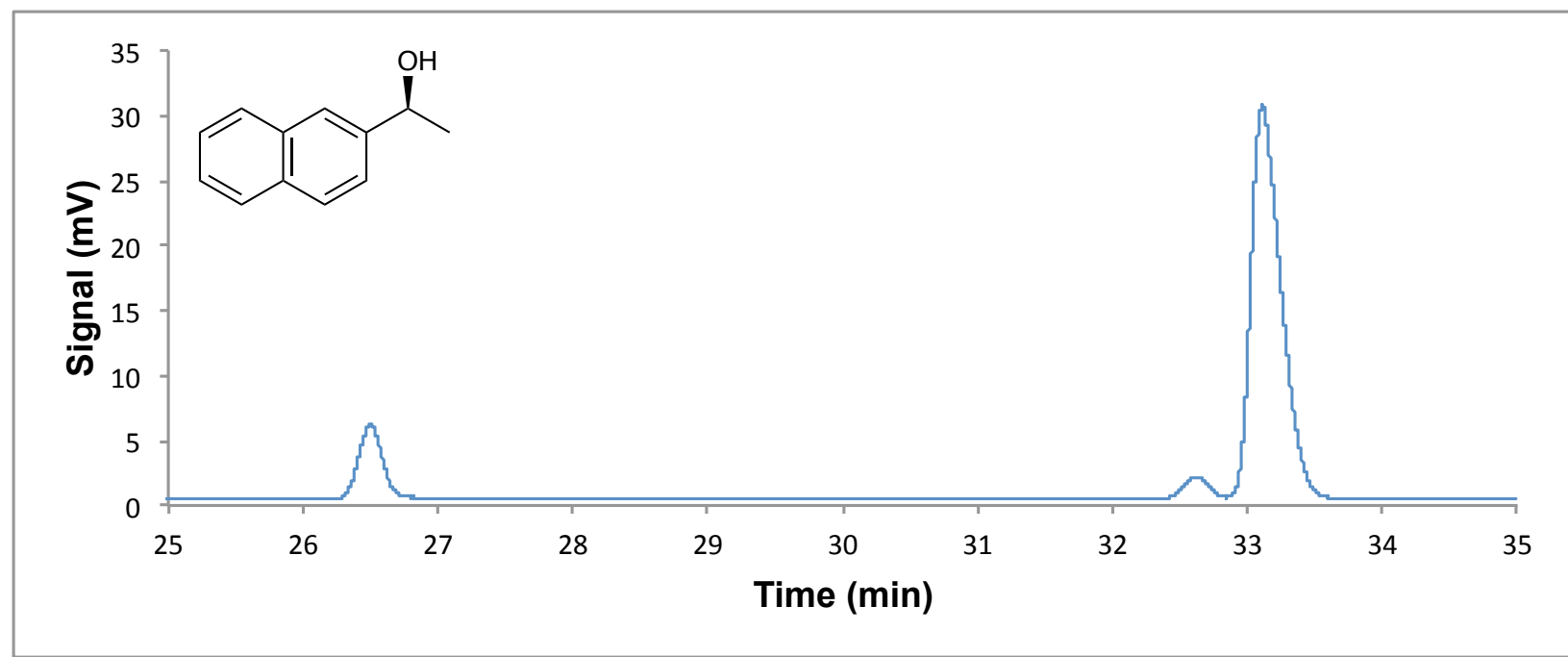

\begin{tabular}{|c|c|c|c|c|c|}
\hline$\#$ & Time & Area & Width & Height & Area \% \\
\hline 1 & 26.496 & 71.74198914 & 5.734248161 & 0.208519012 & 0.584 \\
\hline 2 & 32.621 & 21.57542419 & 1.660766602 & 0.216520727 & 0.176 \\
\hline 3 & 33.111 & 465.0305786 & 30.13682938 & 0.257177323 & 3.787 \\
\hline
\end{tabular}

Figure S39. HPLC chromatogram of the reaction mixture produced by ATH of 2acetylnaphthalene catalyzed by $\mathrm{Cp}{ }^{*} \operatorname{Ir}\left({ }^{\mathrm{D} P L}-\mathrm{C}_{16}\right)$.

$\mathrm{mV}$

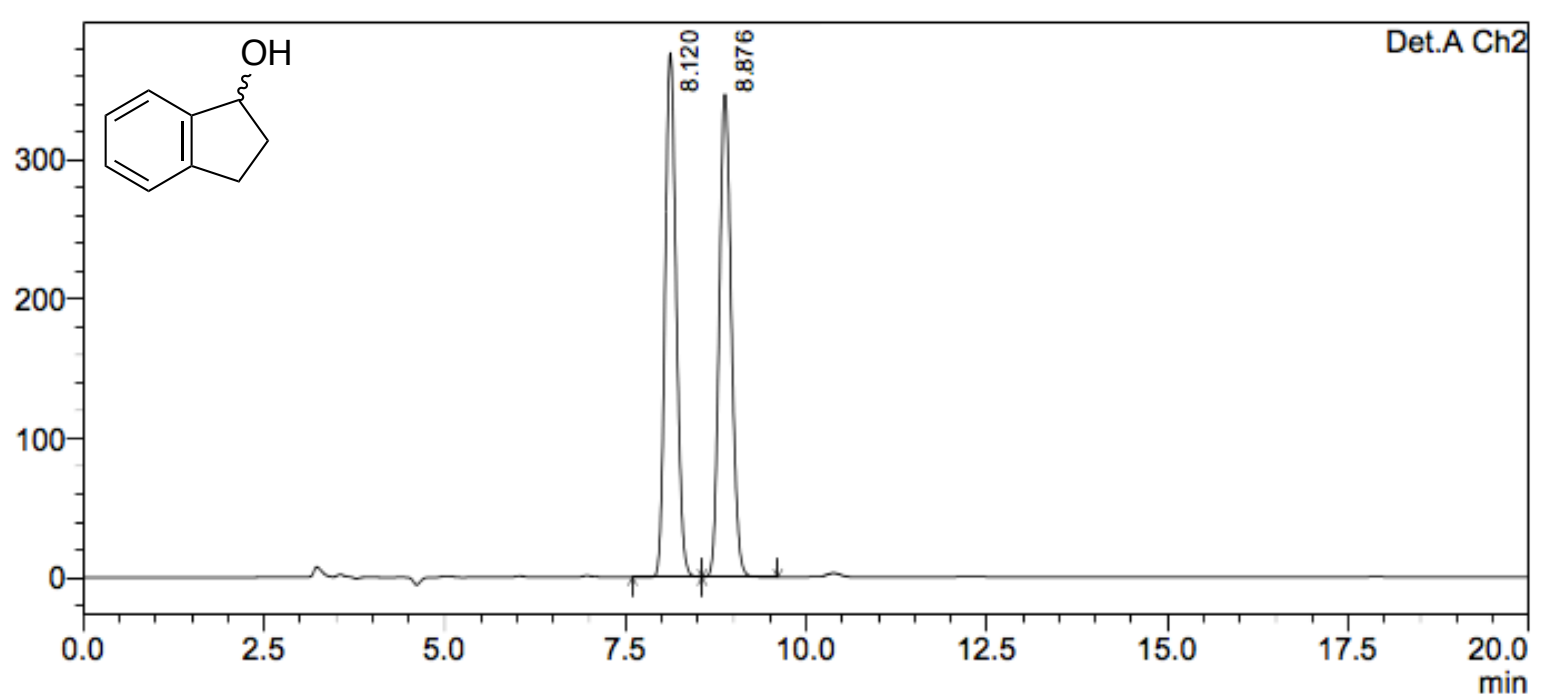

Detector A Ch2 254nm

\begin{tabular}{|r|r|r|r|r|r|}
\hline \multicolumn{1}{|c|}{ Peak\# } & \multicolumn{1}{|c|}{ Ret. Time } & \multicolumn{1}{c|}{ Area } & \multicolumn{1}{c|}{ Height } & \multicolumn{1}{c|}{ Area \% } & \multicolumn{1}{c|}{ Height \% } \\
\hline 1 & 8.120 & 4018406 & 376177 & 49.912 & 52.061 \\
\hline 2 & 8.876 & 4032655 & 346389 & 50.088 & 47.939 \\
\hline Total & & 8051061 & 722566 & 100.000 & 100.000 \\
\hline
\end{tabular}

Figure S40. HPLC chromatogram of the reaction mixture produced by $\mathrm{NaBH}_{4}$ reduction of 1-indanone. 
$\mathrm{mV}$

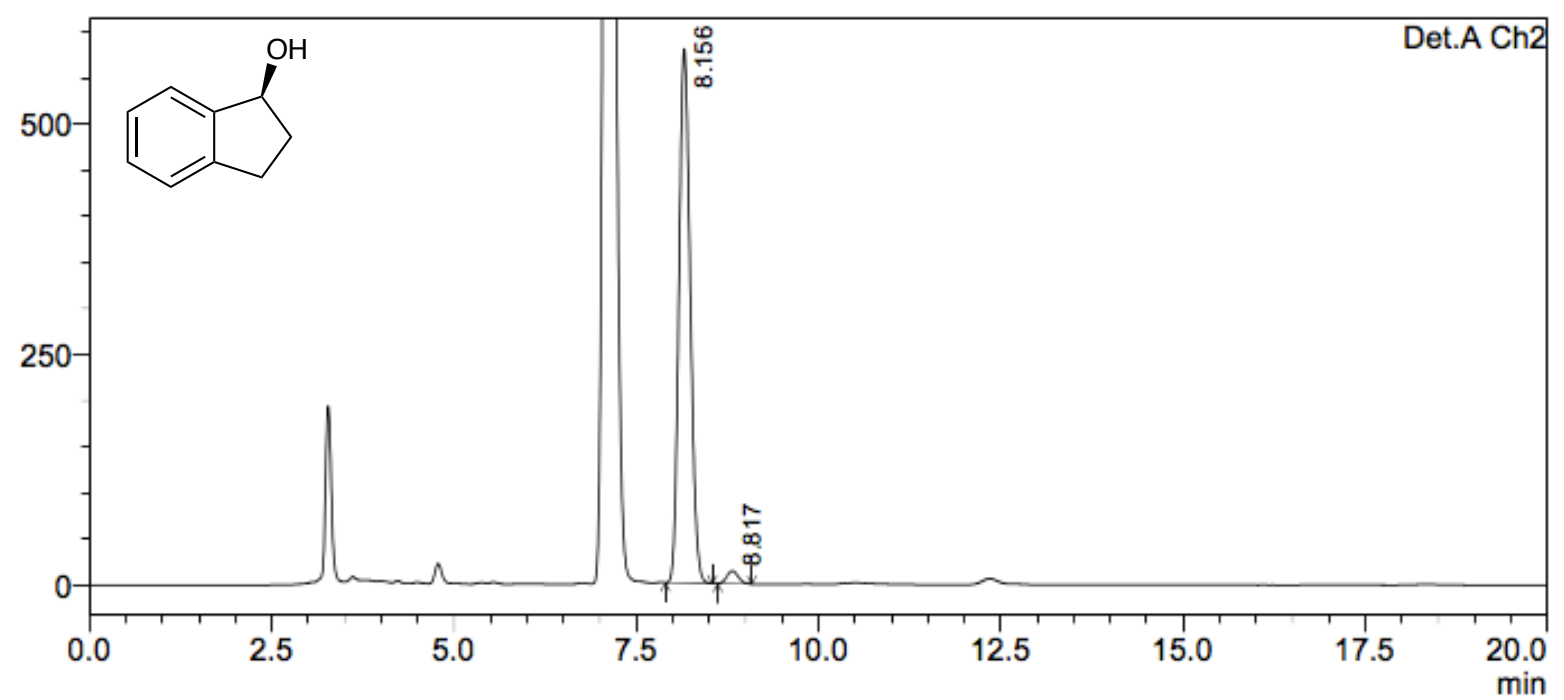

Detector A Ch2 254nm

\begin{tabular}{|r|r|r|r|r|r|}
\hline \multicolumn{1}{|c|}{ Peak\# } & \multicolumn{1}{c|}{ Ret. Time } & \multicolumn{1}{c|}{ Area } & \multicolumn{1}{c|}{ Height } & \multicolumn{1}{c|}{ Area \% } & \multicolumn{1}{c|}{ Height \% } \\
\hline 1 & 8.156 & 6217989 & 579294 & 97.592 & 97.635 \\
\hline 2 & 8.817 & 153407 & 14035 & 2.408 & 2.365 \\
\hline Total & & 6371397 & 593329 & 100.000 & 100.000 \\
\hline
\end{tabular}

Figure S41. HPLC chromatogram of the reaction mixture produced by ATH of 1-indanone catalyzed by $\mathrm{Cp}{ }^{*} \operatorname{Ir}\left({ }^{\mathrm{DPL}}-\mathrm{C}_{16}\right)$.

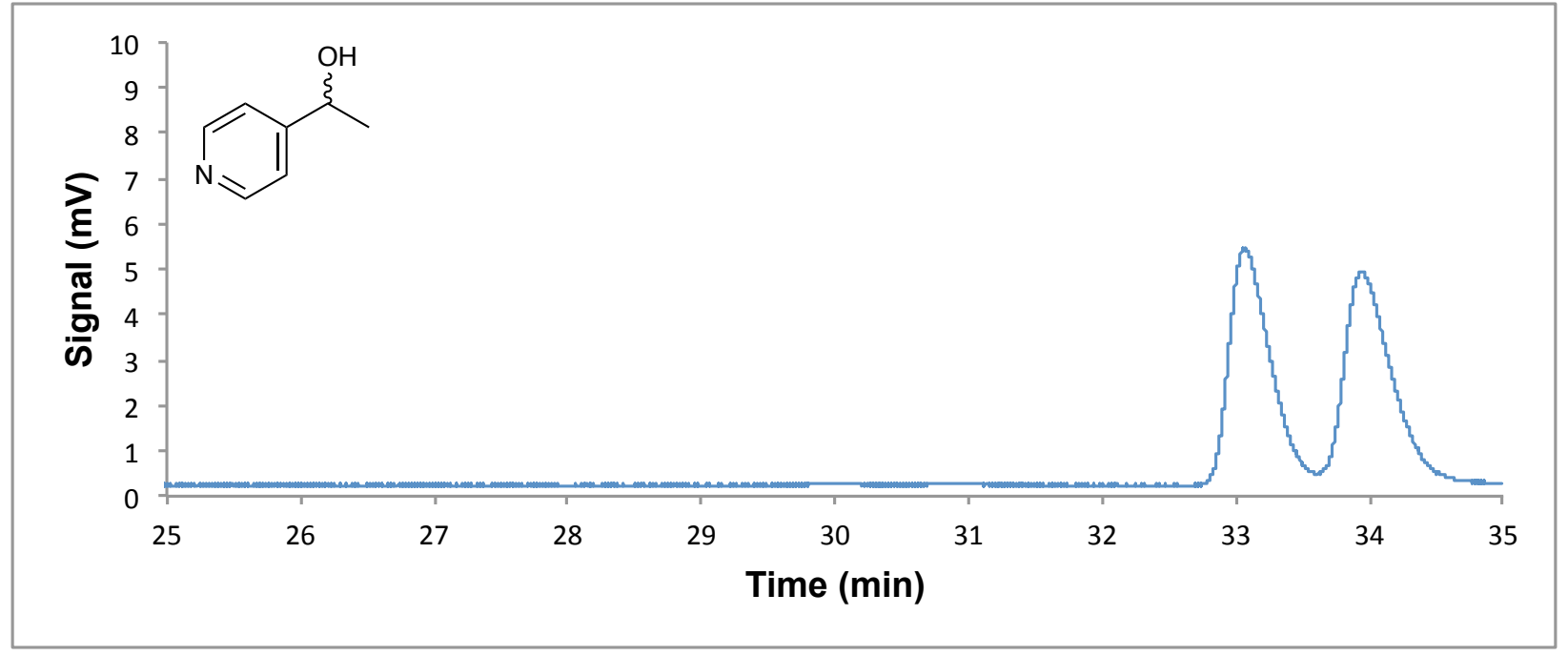

\begin{tabular}{|c|c|c|c|c|c|}
\hline$\#$ & Time & Area & Width & Height & Area \% \\
\hline 1 & 33.063 & 112.4168091 & 5.213475704 & 0.359378934 & 1.587 \\
\hline 2 & 33.936 & 115.3927612 & 4.66753912 & 0.412039995 & 1.629 \\
\hline
\end{tabular}

Figure S42. HPLC chromatogram of the reaction mixture produced by $\mathrm{NaBH}_{4}$ reduction of 4-acetylpyridine. 


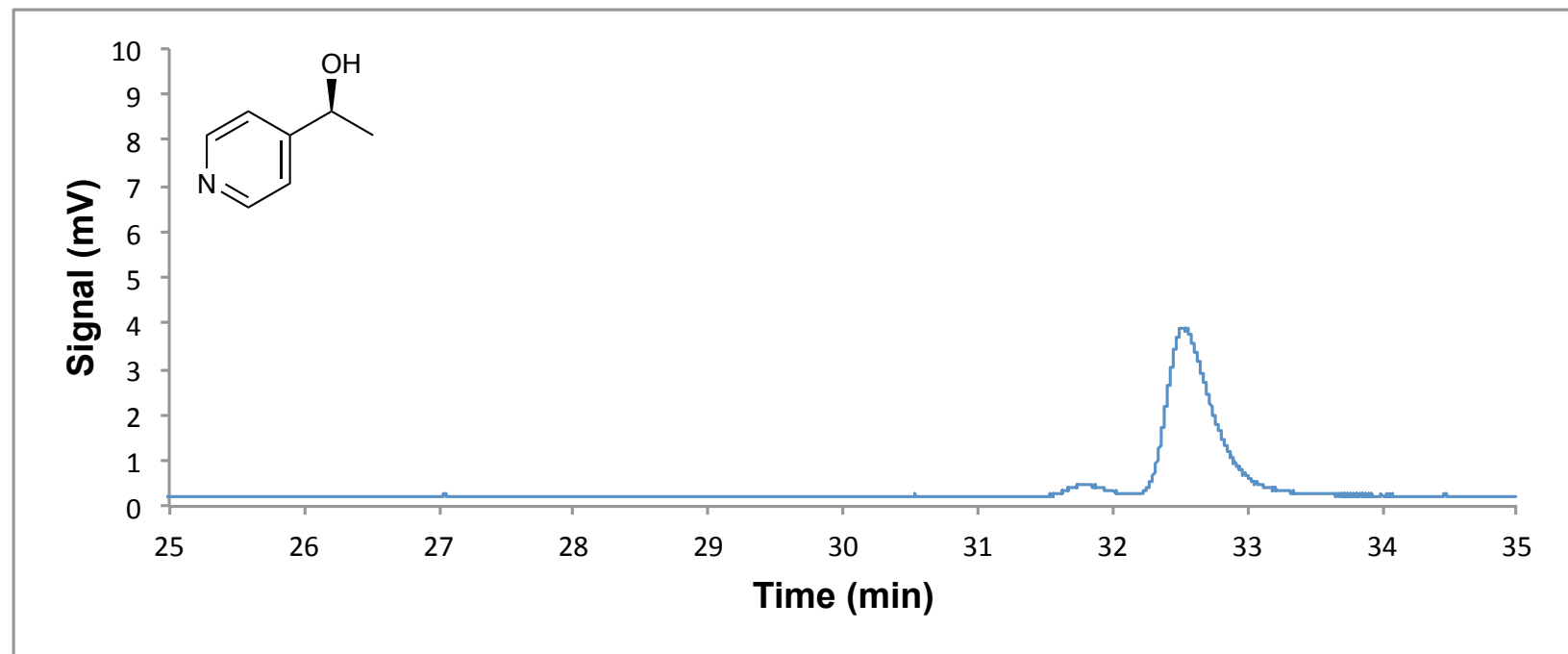

\begin{tabular}{|c|c|c|c|c|c|}
\hline$\#$ & Time & Area & Width & Height & Area \% \\
\hline 1 & 31.802 & 3.954531908 & 0.224936903 & 0.293010443 & 0.041 \\
\hline 2 & 32.527 & 84.02703857 & 3.637865543 & 0.384964913 & 0.864 \\
\hline
\end{tabular}

Figure S43. HPLC chromatogram of the reaction mixture produced by ATH of 4acetylpyridine catalyzed by $\mathrm{Cp}^{*} \operatorname{Ir}\left({ }^{\mathrm{DPL}}-\mathrm{C}_{16}\right)$.

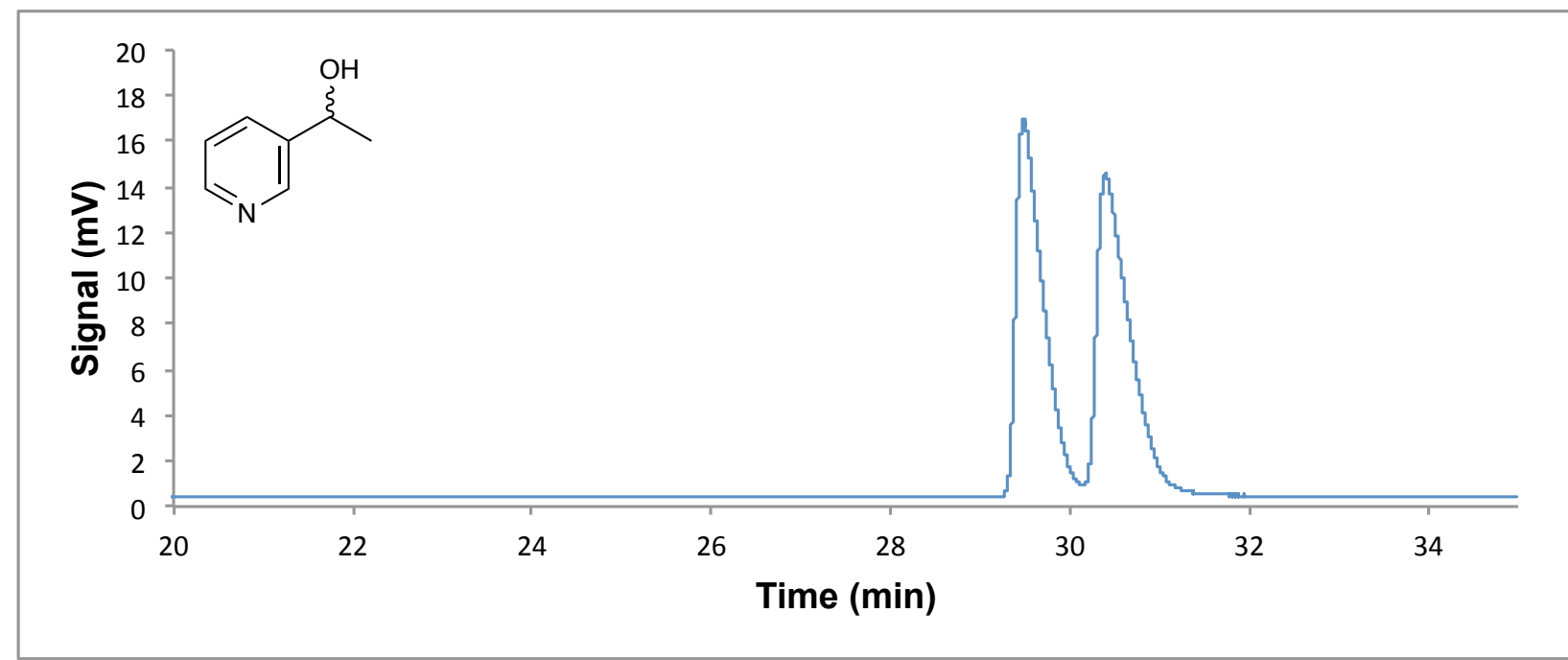

\begin{tabular}{|c|c|c|c|c|c|}
\hline$\#$ & Time & Area & Width & Height & Area \% \\
\hline 1 & 29.479 & 344.8270874 & 16.49756432 & 0.348361611 & 3.224 \\
\hline 2 & 30.395 & 348.189209 & 13.96538258 & 0.41553846 & 3.225 \\
\hline
\end{tabular}

Figure S44. HPLC chromatogram of the reaction mixture produced by $\mathrm{NaBH}_{4}$ reduction of 3-acetylpyridine. 


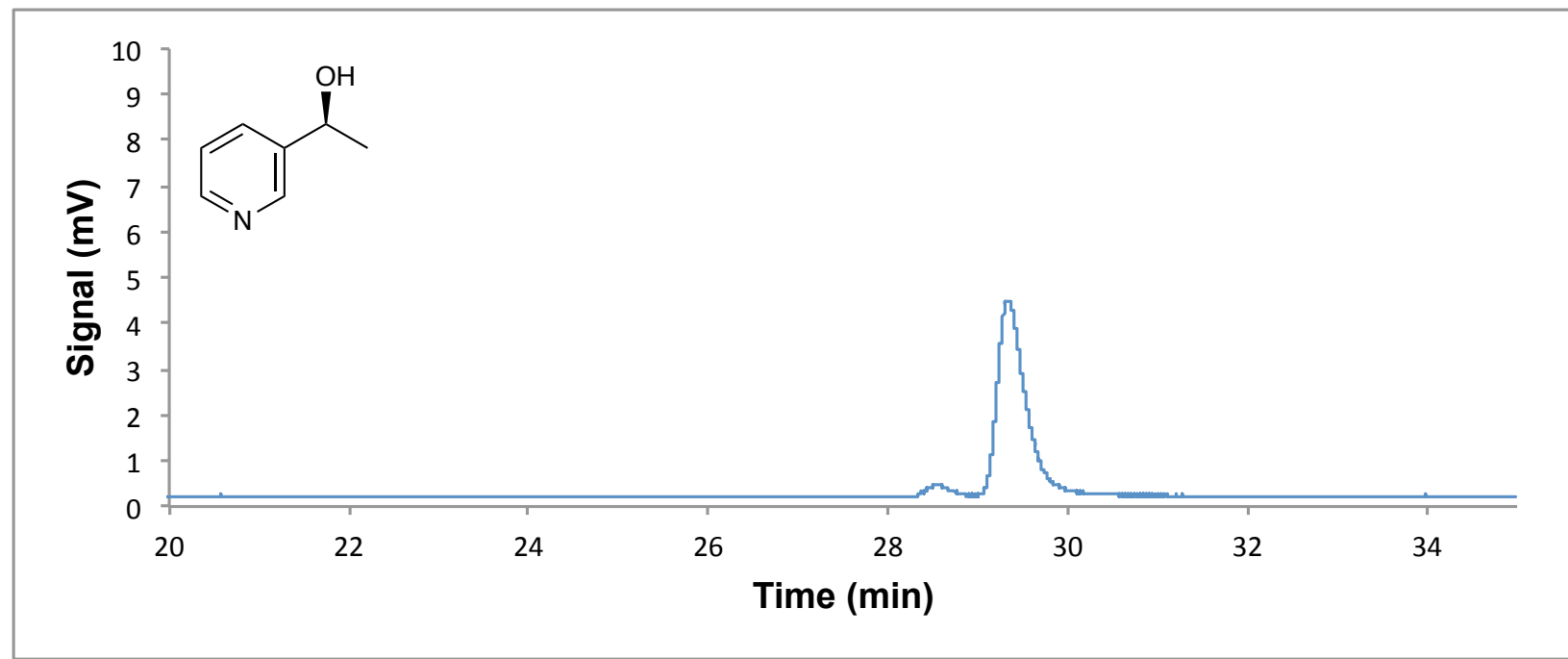

\begin{tabular}{|c|c|c|c|c|c|}
\hline$\#$ & Time & Area & Width & Height & Area \% \\
\hline 1 & 28.567 & 4.492972851 & 0.25648573 & 0.291957289 & 0.044 \\
\hline 2 & 29.334 & 91.61925507 & 4.258590698 & 0.358566403 & 0.895 \\
\hline
\end{tabular}

Figure S45. HPLC chromatogram of the reaction mixture produced by ATH of 3acetylpyridine catalyzed by $\mathrm{Cp}{ }^{*} \operatorname{Ir}\left({ }^{\mathrm{DPL}}-\mathrm{C}_{16}\right)$.

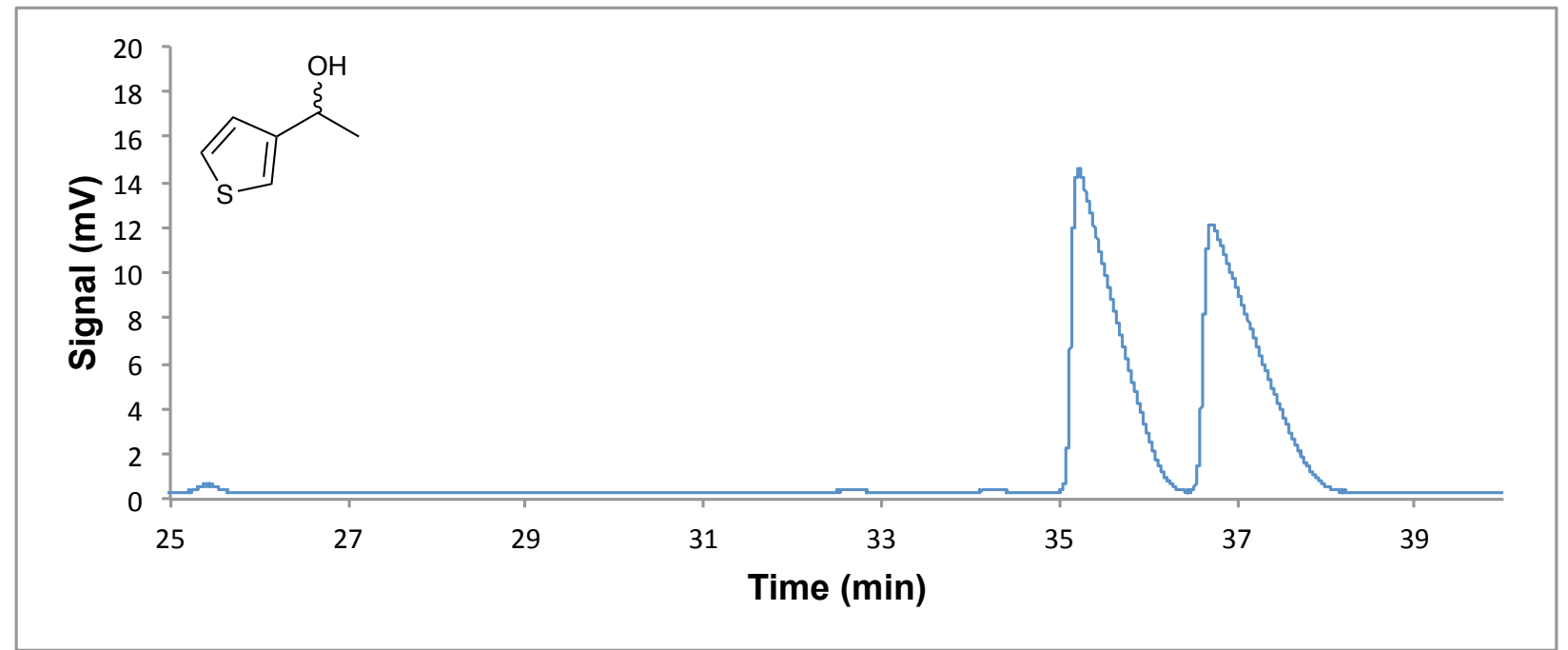

\begin{tabular}{|c|c|c|c|c|c|}
\hline$\#$ & Time & Area & Width & Height & Area \% \\
\hline 1 & 25.428 & 5.850280285 & 0.350262552 & 0.278375953 & 0.056 \\
\hline 2 & 35.217 & 483.9571533 & 14.23548985 & 0.566608727 & 4.592 \\
\hline 3 & 36.714 & 487.9306946 & 11.77091122 & 0.690870702 & 4.630 \\
\hline
\end{tabular}

Figure S46. HPLC chromatogram of the reaction mixture produced by $\mathrm{NaBH}_{4}$ reduction of 3-acetylthiophene. 


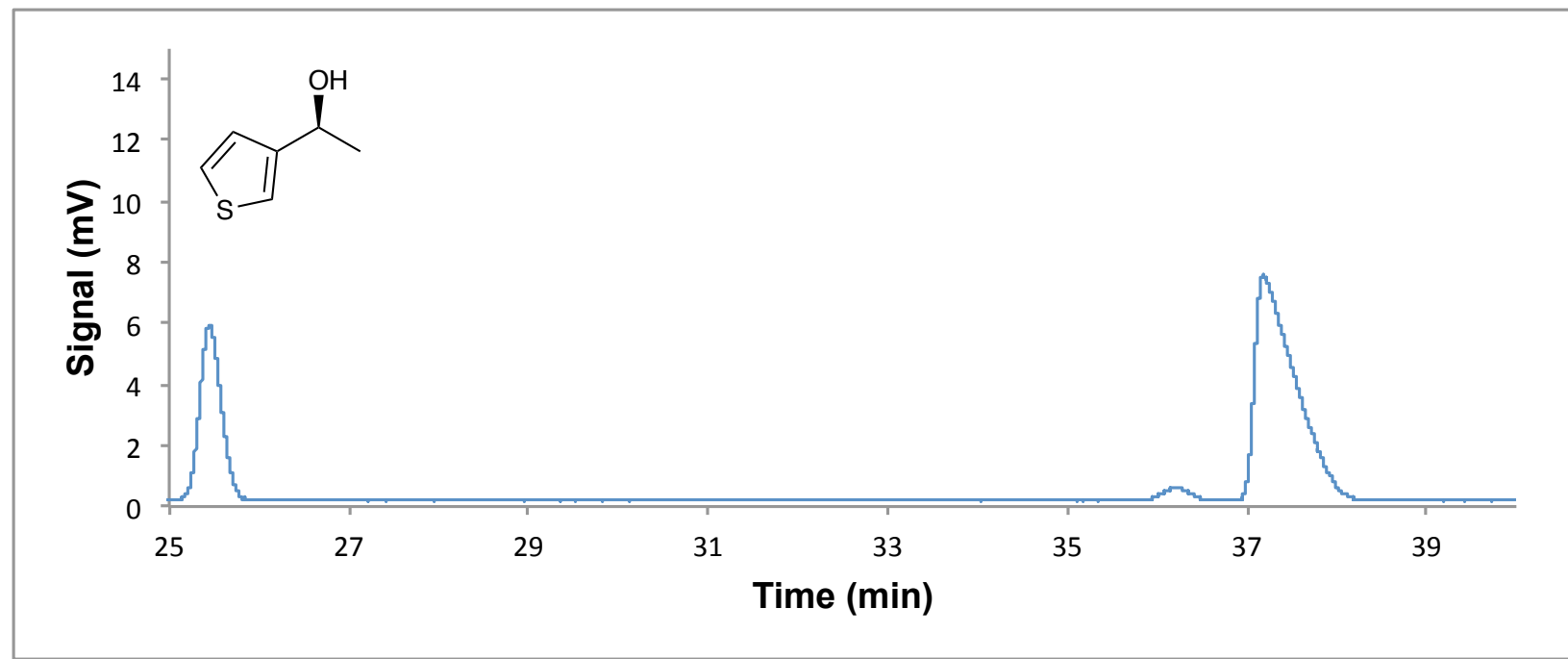

\begin{tabular}{|c|c|c|c|c|c|}
\hline$\#$ & Time & Area & Width & Height & Area \% \\
\hline 1 & 25.449 & 97.25334167 & 5.766941071 & 0.281065643 & 0.896 \\
\hline 2 & 36.207 & 9.474720001 & 0.442512959 & 0.356852829 & 0.087 \\
\hline 3 & 37.188 & 231.0188904 & 7.332841873 & 0.525078118 & 2.128 \\
\hline
\end{tabular}

Figure S47. HPLC chromatogram of the reaction mixture produced by ATH of 3acetylthiophene catalyzed by $\mathrm{Cp}{ }^{*} \operatorname{Ir}\left({ }^{\mathrm{DPL}}-\mathrm{C}_{16}\right)$.

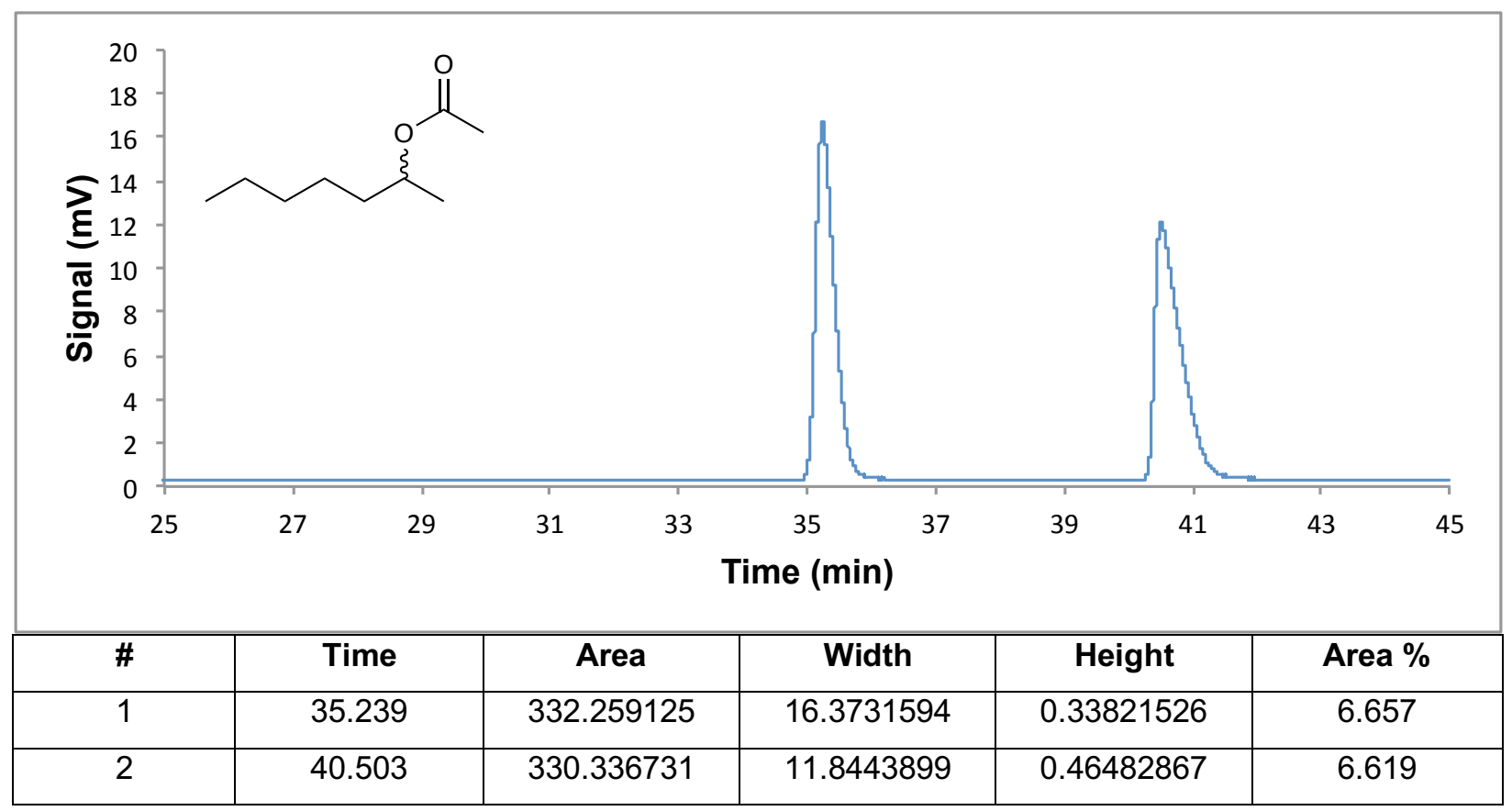

Figure S48. HPLC chromatogram of the reaction mixture produced by $\mathrm{NaBH}_{4}$ reduction of 2-heptanone and subsequent acetylation. 


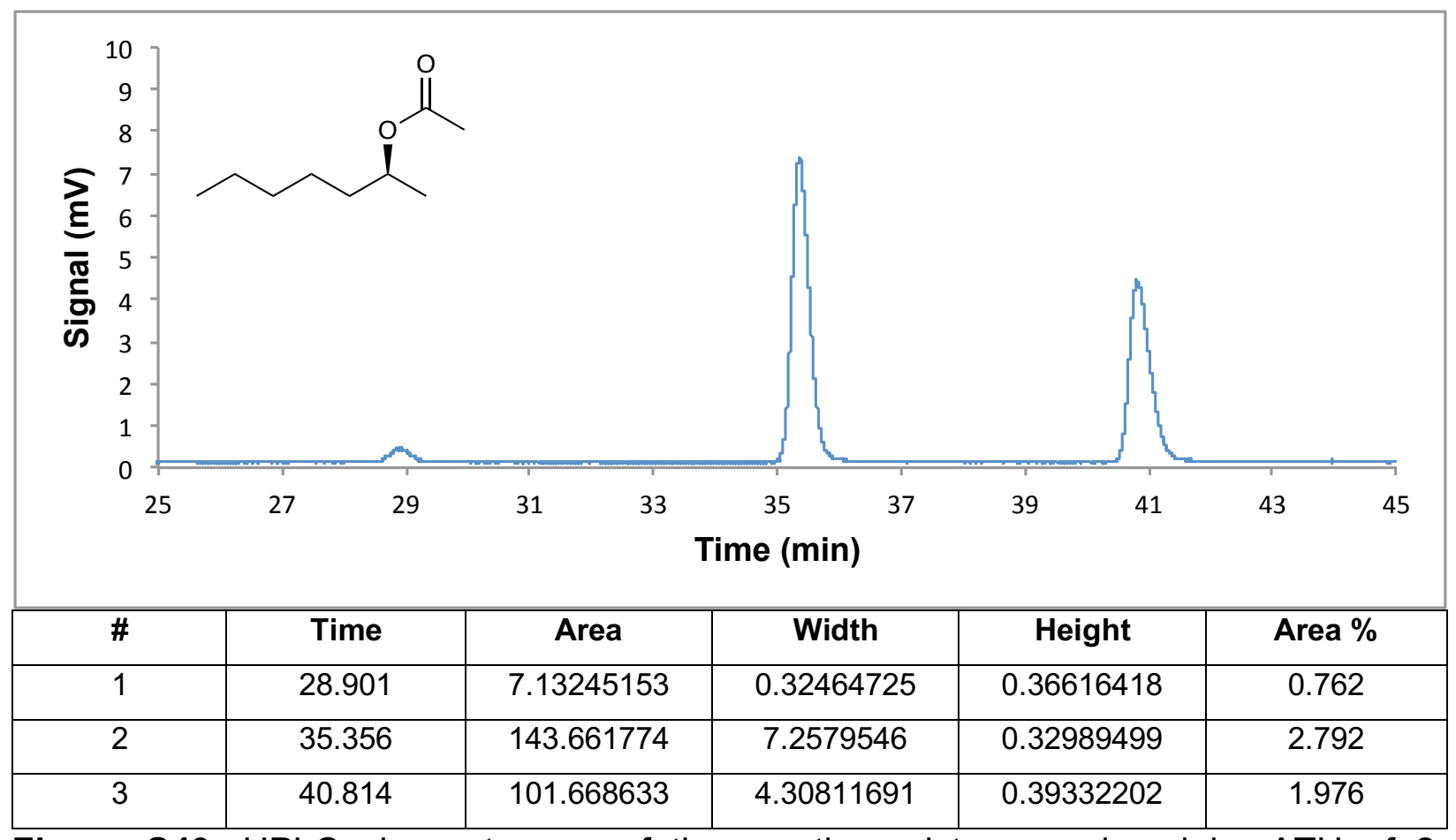

Figure S49. HPLC chromatogram of the reaction mixture produced by ATH of 2heptanone catalyzed by $\mathrm{Cp}{ }^{*} \operatorname{Ir}\left({ }^{\mathrm{DPL}}-\mathrm{C}_{16}\right)$ and subsequent acetylation. 
Table S2. Crystallographic parameters for ${ }^{\mathrm{DPF}}-\mathrm{C}_{12}$

\begin{tabular}{|c|c|}
\hline formula & $\mathrm{C}_{26} \mathrm{H}_{43} \mathrm{~N}_{3} \mathrm{O}_{2}$ \\
\hline formula weight $\left(\mathrm{M}_{\mathrm{r}}\right)$ & 429.63 \\
\hline Crystal system, space group & Monoclinic, $P 2_{1}$ \\
\hline$a, \AA$ & $9.056(5)$ \\
\hline $\mathrm{b}, \AA$ & $4.839(5)$ \\
\hline$c, \AA$ & $28.594(5)$ \\
\hline$\alpha,{ }^{\circ}$ & 90 \\
\hline$\beta,{ }^{\circ}$ & $97.735(5)$ \\
\hline$\gamma,{ }^{\circ}$ & 90 \\
\hline $\mathrm{V}, \AA^{3}$ & $1241.6(15)$ \\
\hline Z & 2 \\
\hline$\rho_{\text {calcd }}\left(\mathrm{g} \mathrm{cm}^{-3}\right)$ & 1.149 \\
\hline absorp. coeff. $\mu\left(\mathrm{mm}^{-1}\right)$ & 0.072 \\
\hline temp, $\mathrm{K}$ & 100 \\
\hline total no. data & 4041 \\
\hline no. unique data & 2292 \\
\hline $\mathrm{R}, \%$ & 0.046 \\
\hline wR2, \% & 0.1167 \\
\hline no. of parameters & 292 \\
\hline max/min peaks, e/Á & $0.21 /-0.26$ \\
\hline
\end{tabular}




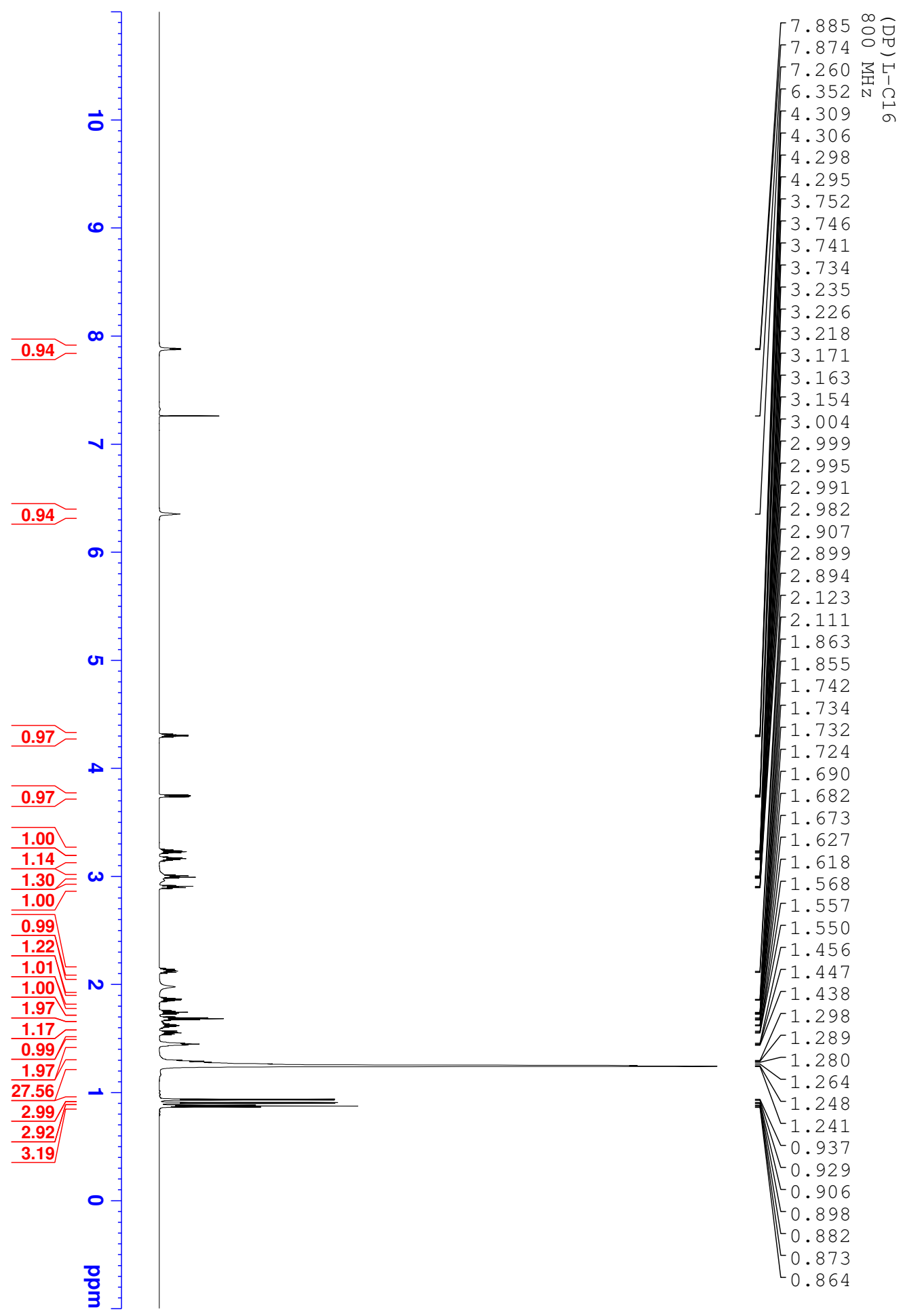

Figure S50. ${ }^{1} \mathrm{H}(800 \mathrm{MHz}) \mathrm{NMR}$ of ${ }^{\mathrm{DPL}-\mathrm{C}_{16}}$ 


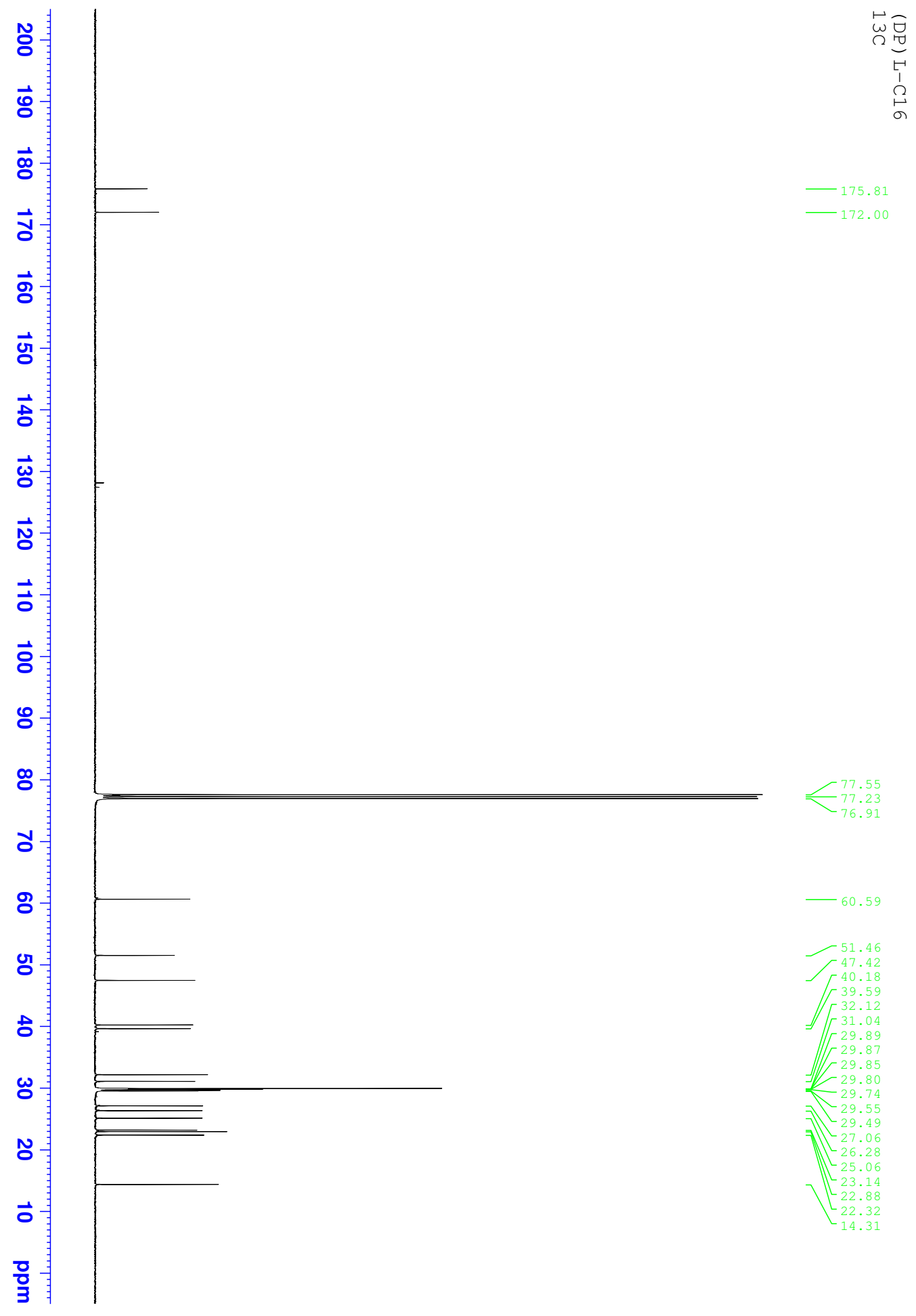

Figure S51. ${ }^{13} \mathrm{C}(100 \mathrm{MHz}) \mathrm{NMR}$ of ${ }^{\mathrm{DPL}-\mathrm{C}_{16}}$ 


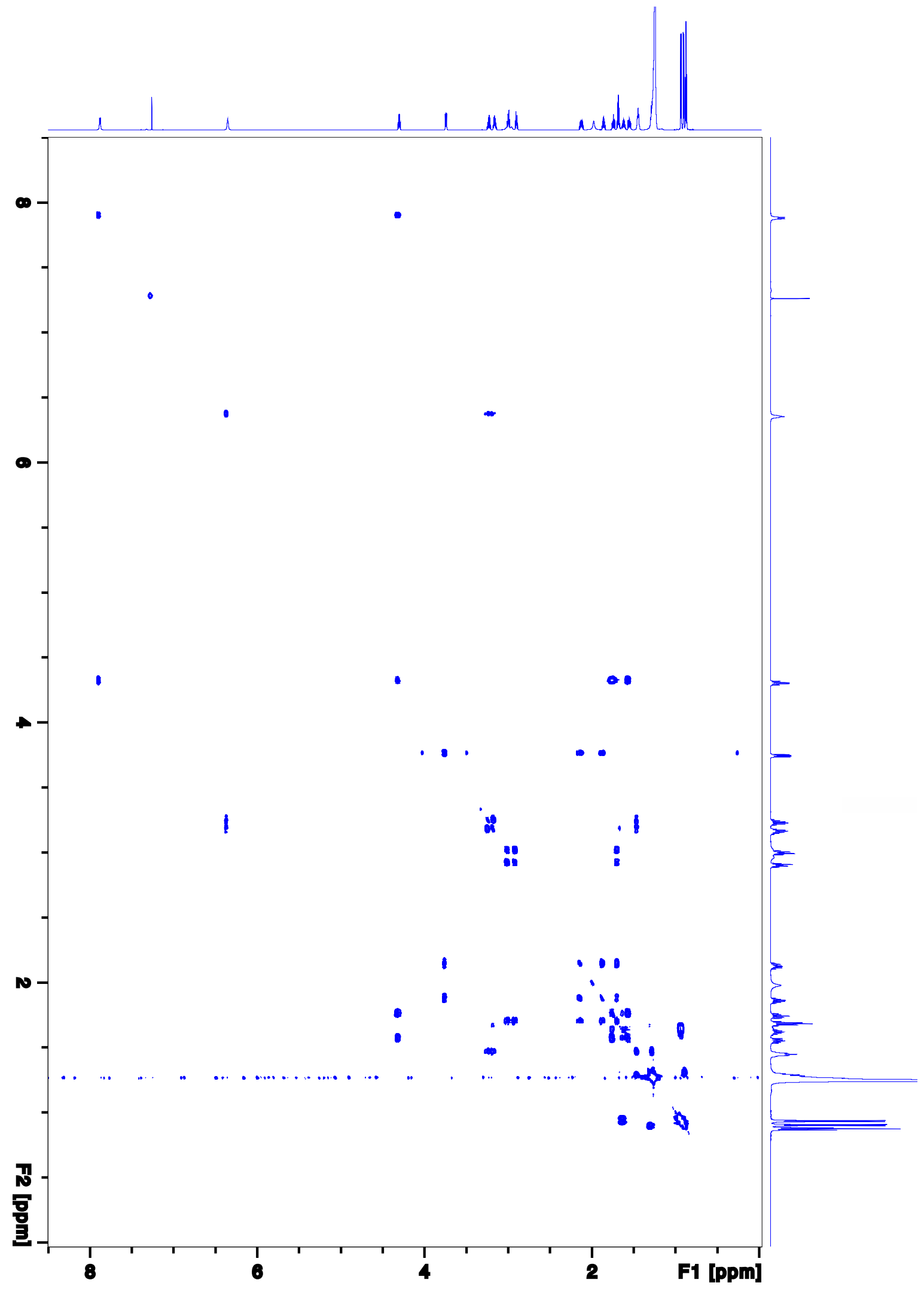

Figure S52. 2D-COSY (800 MHz) NMR of ${ }^{\mathrm{DPL}-\mathrm{C}_{16}}$ 


\section{References:}

(1) Frisch, M. J.; Trucks, G. W.; Schlegel, H. B.; Scuseria, G. E.; Robb, M. A.; Cheeseman, J. R.; Scalmani, G.; Barone, V.; Petersson, G. A.; Nakatsuji, H.; Li, X; Caricato, M.; Marenich, A. V.; Bloino J.; Janesko, B. G.; Gomperts, R.; Mennucci, B.; Hratchian H. P.; Ortiz, J. V.; Izmaylov, A. F.; Sonnenberg, J. L.; et al. Gaussian 16 Rev. B.01, Wallingford, CT, 2016

(2) Adamo, C.; Barone, V., Toward Reliable Density Functional Methods Without Adjustable Parameters: The PBE0 model. J. Chem. Phys. 1999, 110, 6158-6170.

(3) Weigend, F.; Ahlrichs, R., Balanced Basis Sets of Split Valence, Triple Zeta Valence and Quadruple Zeta Valence Quality for $\mathrm{H}$ to Rn: Design and Assessment of Accuracy. Phys. Chem. Chem. Phys. 2005, 7, 3297-3305.

(4) Lengyel, Z.; Rufo, C. M.; Korendovych, I. V. In Peptide Self-Assembly: Methods and Protocols; Nilsson B. L., Doran T. M., Eds.; Springer New York: New York, 2018; pp 261-270.

(5) Ling, F.; Nian, S.; Chen, J.; Luo, W.; Wang, Z.; Lv, Y.; Zhong, W. Development of Ferrocene-Based Diamine-Phosphine-Sulfonamide Ligands for Iridium-Catalyzed Asymmetric Hydrogenation of Ketones. J. Org. Chem. 2018, 83, 10749-10761.

(6) Schiwek, C. H.; Vasilenko, V.; Wadepohl, H.; Gade, L. H. The Open d-Shell Enforces the Active Space in 3d Metal Catalysis: Highly Enantioselective Chromium(II) Pincer Catalysed Hydrosilation of Ketones. Chem. Commun. 2018, 54, 9139-9142.

(7) Cettolin, M.; Puylaert, P.; Pignataro, L.; Hinze, S.; Gennari, C.; de Vries, J. G. Use of the Trost Ligand in the Ruthenium-Catalyzed Asymmetric Hydrogenation of Ketones ChemCatChem 2017, 9, 3125-3130.

(8) Margalef, J.; Slagbrand, T.; Tinnis, F.; Adolfsson, H.; Diéguez, M.; Pàmies, O. ThirdGeneration Amino Acid Furanoside-Based Ligands from D-Mannose for the Asymmetric Transfer Hydrogenation of Ketones: Catalysts with an Exceptionally Wide Substrate Scope. Adv. Synth. Catal. 2016, 358, 4006-4018.

(9) Hodgkinson, R.; Jurčík, V.; Zanotti-Gerosa, A.; Nedden, H. G.; Blackaby, A.; Clarkson, G. J.; Wills, M. Synthesis and Catalytic Applications of an Extended Range of Tethered Ruthenium(II) $/ \eta^{6}$-Arene/Diamine Complexes. Organometallics 2014, 33, 5517-5524.

(10) Soni, R.; Jolley, K.E.; Clarkson, G. J.; Wills, M. Direct Formation of Tethered Ru(II) Catalysts Using Arene Exchange. Org. Lett. 2013, 15, 5110-5113. 